Radial Basis Function Approximation

- Radial Basis Function Networks

\section{Radial Basis Function Networks}

Martin D. Buhmann

Justus-Liebig University, Gießen, Germany

\section{Synonyms}

Kernel methods; Networks with kernel functions; Neural networks; Quasi-interpolation; Radial basis function approximation; Radial basis function neural networks; Regularization networks; Support vector machines

\section{Definition}

Radial basis function networks are a means of approximation by algorithms using linear combinations of translates of a rotationally invariant function, called the radial basis function. The coefficients of these approximations usually solve a minimization problem and can also be computed by interpolation processes. Sometimes the very useful approach of quasi-interpolation is also applied where approximations are computed that do not necessarily match the target functions pointwise but satisfy certain smoothness and decay conditions. The radial basis functions constitute so-called reproducing kernels on certain Hilbert spaces or - in a slightly more general setting - semi-Hilbert spaces. In the latter case, the aforementioned approximation also contains an element from the null-space of the seminorm of the semi-Hilbert space. That is usually a polynomial space.

\section{Motivation and Background}

Radial basis function networks are a method to approximate functions and data in a way which is related to the idea of neural networks and learning with kernels. More specifically, approximations of functions or data via algorithms that make use of networks (or neural networks) can be interpreted as either interpolation or minimization problems using kernels of certain shapes, called radial basis functions in the form in which we wish to consider them in this entry. In all cases, they are usually high-dimensional approximations, that is, the number of unknowns $n$ in the argument of the kernel may be very large. On the other hand, the number of learning examples ("data") may be quite small. The name neural networks comes from the idea that this learning process simulates the natural functioning of neurons.

At any rate, the purpose of this approach will be the modelization of the learning process by mathematical methods. In most practical cases of 
networks, the data from which we will learn in the method are rare, i.e., we have few data "points." We will consider this learning approach as an approximation problem in this description; essentially it is a minimizing (regression) problem.

\section{Structure of the Network/Learning System}

To begin with, let $\varphi: \mathbb{R}_{+} \rightarrow \mathbb{R}$ be a univariate continuous function and $\|\cdot\|$ be the Euclidean norm on $\mathbb{R}^{n}$ for some $n \in \mathbb{N}$, as used for approximation in the seminal paper by Schoenberg (1938). Here, $\mathbb{R}_{+}$denotes the set of nonnegative reals. Therefore

$$
\begin{aligned}
\varphi(\|\cdot\|): \mathbb{R}^{n} & \rightarrow \mathbb{R}, \\
\left(x_{1}, x_{2}, \ldots, x_{n}\right)^{T} & \mapsto \varphi\left(\sqrt{x_{1}^{2}+x_{2}^{2}+\cdots+x_{n}^{2}}\right),
\end{aligned}
$$

is a multivariate function and here the number $n$ of unknowns may be very large in practice. This function is rotationally invariant. Incidentally, much of what is going to be said here will work if we replace this function by a general, $n$ variate function which needs no longer be rotationally invariant, but then, strictly speaking, we are no longer talking about radial basis functions. Then other conditions may replace the restriction to radiality. Nonetheless we stick to the simple case (which is entirely sufficient for many practical applications) when the function really is radially symmetric.

We also require for the time being that this $n$ variate function be positive definite, that is, for all finite sets $\Xi$ of pairwise different so-called centers or data sites $\xi \in \Xi \subset \mathbb{R}^{n}$, the symmetric matrix

$$
A=\{\varphi(\|\xi-\zeta\|)\}_{\xi, \xi \in \Xi}
$$

is a positive definite matrix. The condition of pairwise different data in $\Xi$ may of course in practice not be necessarily met. For quasiinterpolation, no linear systems need to be solved that depend on the target data, but other conditions that guarantee localness and polynomial accuracy of the approximants are required.

This property is usually obtained by requiring that $\varphi(\|\cdot\|)$ be absolutely integrable, and its Fourier transform - which thereby exists and is continuous - is positive everywhere ("Bochner's theorem"). An example for such a useful function is the exponential (the "Gauß-kernel") $\varphi(r)=$ $\exp \left(-c^{2} r^{2}\right), r \geq 0$, where $c$ is a positive parameter. For this the above positive definiteness is guaranteed for all positive $c$ and all $n$. Another example is the Poisson-kernel $\varphi(r)=\exp (-c r)$. However, we may also take the non-integrable "inverse multiquadrics" $\varphi(r)=1 / \sqrt{r^{2}+c^{2}}$ which has a Fourier transform in the generalized or distributional sense that is also positive everywhere except at zero. There it has a singularity. Nonetheless, the aforementioned matrices of the form $A$ are still always positive definite for these exponentials and the inverse multiquadrics so long as $c>0$ and $n=1,2, \ldots$ Still further examples come from the so-called Dagum class of radial basis functions $\varphi(r)=1-\left(r^{\beta} /\left(1+r^{\beta}\right)\right)^{\gamma}$ which give positive definiteness for a variety of choices of parameters $\beta$ and $\gamma$.

This requirement of positive definiteness guarantees that for all given finite sets $\Xi$ and "data" $f_{\xi} \in \mathbb{R}, \xi \in \Xi$, there is a unique linear combination:

$$
s(x)=\sum_{\xi \in \Xi} \lambda_{\xi} \varphi(\|x-\xi\|), \quad x \in \mathbb{R}^{n},
$$

which satisfies the linear interpolation conditions:

$$
s(\xi)=f_{\xi}, \quad \forall \xi \in \Xi .
$$

This is because the interpolation matrix which is used to compute the coefficients $\lambda_{\xi}$ is just the matrix $A$ above which is positive definite, thus regular. The expression in the penultimate display is the network that approximates the data given by the user. Of course the interpolation conditions are just what is meant by learning from examples, the data being the $|\Xi|$ examples. Here as always, $|\Xi|$ denotes the cardinality of the set $\Xi$. In the learning theory, the linear space spanned by the 
above translates of $\varphi(\|\cdot\|)$ by $\xi \in \Xi$ is called the feature space with $\varphi$ as activation function.

Incidentally, it is straightforward to generalize the approximation method to an approximation to data in $\mathbb{R}^{m}, m \in \mathbb{N}$, by approximating the data $f_{\xi} \in \mathbb{R}^{m}$ componentwise by $m$ such expressions as the above, call them $s_{1}, s_{2}, \ldots, s_{m}$.

\section{Applications}

Applications include classification of data, pattern recognition, time series analysis, picture smoothing similar to diffusion methods, and optimization.

\section{Theory/Solution}

Returning to interpolation, the problem may also be reinterpreted as a minimization problem. If we define the weighted $L^{2}$-integral

$$
\|g\|_{\varphi}:=\frac{1}{(2 \pi)^{n / 2}} \sqrt{\int_{\mathbb{R}^{n}} \frac{1}{\hat{\varphi}(\|x\|)}|\hat{g}(x)|^{2} d x}
$$

with $\hat{\varphi}$ still being the above positive Fourier transform, for all $g: \mathbb{R}^{n} \rightarrow \mathbb{R}$ for which the Fourier transform in the sense of $L^{2}\left(\mathbb{R}^{n}\right)$ is well defined and for which the above integral is finite, we may ask for the approximant to the above data - which still must satisfy the aforementioned interpolation conditions - that minimizes $\|\cdot\|_{\varphi}$. As Duchon noted, for example, for the thin-plate spline case $\varphi(r)=r^{2} \log r$ in this seminal paper, this is just the above interpolant, i.e., that linear combination $s$ of translates of radial basis functions, albeit in the thin-plate spline case with a linear polynomial added as we shall see below.

This works immediately both for the two examples of exponential functions and the inverse multiquadrics. Note that the fact that the latter has a Fourier transform with a singularity at the origin does not matter since its reciprocal appears as a weight function in the integral above. The important requirement is that the Fourier transform has no zero. It also works for the positive definite radial basis functions of compact support, for instance, in Buhmann (1998).

\section{Regularization and Generalizations}

Since, generally, the interpolation problem to data may be ill conditioned or unsuitable in the face of data errors, smoothing or regularization is appropriate as an alternative. Indeed, the interpolation problem may be replaced by a smoothing problem which is of the form

$$
\frac{1}{|\Xi|} \sum_{\xi \in \Xi}\left(s(\xi)-f_{\xi}\right)^{2}+\mu\|s\|_{\varphi}^{2}=\min _{s} !
$$

Here the $L^{2}$-integral is still the one used in the description above and $\mu$ is a positive smoothing parameter.

However, when there is only a trivial nullspace of the $\|\cdot\|_{\varphi}$, i.e., $g=0$ is the only $g$ with $\|g\|_{\varphi}=0$, then it is a norm, and the solution of this problem will have the form

$$
s(x)=\sum_{\xi \in \Xi} \lambda_{\xi} \varphi(\|x-\xi\|), \quad x \in \mathbb{R}^{n} .
$$

This is where the name regularization network comes from, regularization and smoothing being used synonymously. The form used in the penultimate display is a classical regularizing network problem or in the spline terminology a smoothing spline problem. For so-called support vector machines, the square of the residual term $s(\xi)-f_{\xi}$ should be replaced by another expression, for example, the one by Vapnik (1996):

$\left|s(\xi)-f_{\xi}\right|_{\varepsilon}:= \begin{cases}f_{\xi}-s(\xi)-\varepsilon & \text { if }\left|f_{\xi}-s(\xi)\right| \geq \varepsilon \\ 0 & \text { otherwise }\end{cases}$

and for the support vector machines classification by the truncated power function $(\cdot)_{+}^{v}$ which is a positive power for positive argument and otherwise zero.

In the case of a classical regularizing network, the coefficients of the solution may be found by solving a similar linear system to the 
standard interpolation linear system mentioned above, namely,

$$
(A+\mu I) \lambda=f
$$

where $f$ is the vector $\left(f_{\xi}\right)_{\xi \in \Xi}$ in $\mathbb{R}^{\Xi}$ of the data given and $\lambda=\left(\lambda_{\xi}\right)_{\xi \in \Xi}$. The $I$ denotes the $|\Xi| \times$ $|\Xi|$ identity matrix and $A$ is still the same matrix as above. Incidentally, also scaling mechanisms may be introduced into the radial basis function by replacing the simple translate $\varphi(\|x-\xi\|)$ by $\varphi(\|x-\xi\| / \delta)$ for a positive $\delta$ which may even depend on $\xi$.

The ideas of regularization and smoothing are of course not new; for instance, regularization goes back to Tichonov and Arsenin (1977) ("Tichonov regularization") and spline smoothing to Wahba (1985), especially when the smoothing parameter is adjusted via cross-validation or GCV (generalized cross-validation).

Now to the case of semi-norms $\|\cdot\|_{\varphi}$ with nontrivial null-spaces: indeed, the same idea can be carried through for other radial basis functions as well. In particular we are thinking here of those ones that do not provide positive definite radial basis interpolation matrices but strictly conditionally positive definite ones. We have strictly positive definite radial basis functions of order $k+1, k \geq-1$, if the above interpolation matrices $A$ are still positive definite but only on the subspace of those nonzero vectors $\lambda=\left(\lambda_{\xi}\right)$ in $\mathbb{R}^{\Xi}$ which satisfy

$$
\sum_{\xi \in \Xi} \lambda_{\xi} p(\xi)=0 \quad \forall p \in \mathbb{P}_{n}^{k}
$$

where $\mathbb{P}_{n}^{k}$ denotes the linear space of polynomials in $n$ variables with total degree at most $k$. In other words, the quadratic form $\lambda^{T} A \lambda$ need only be positive for such $\lambda \neq 0$. For simplicity of the presentation, we shall let $\mathbb{P}_{n}^{-1}$ denote $\{0\}$. In particular, if the radial basis function is conditionally positive definite of order 0 , its interpolation matrices $A$ are always positive definite, that is, without condition. Also, we have the minimal requirement that the sets of centers $\Xi$ are unisolvent for this polynomial space, i.e., the only polynomial $p \in \mathbb{P}_{n}^{k}$ that vanishes identically on $\Xi$ is the zero polynomial.

The connection of this with a layered neural network is that the approximation above is a weighted sum (weighted by the coefficients $\lambda_{\xi}$ ) over usually nonlinear activation functions $\varphi$. The entries in the sum are the radial basis function neurons and there are usually many of them. The number of nodes in the model is $n$. The hidden layer of "radial basis function units" consists of $|\Xi|$ nodes, i.e., the number of centers in our radial basis function approximation. The output layer has $m$ responses if the radial basis function approximation above is generalized to $m$-variate data, i.e., then we get $s_{1}, s_{2}, \ldots, s_{m}$ instead of just $s$, as already described. This network here is of the type of a nonlinear, layered, feedforward network. More than one hidden layer is unusual. The choice of the radial basis functions (its smoothness, for instance) and the flexibility in the positioning of the centers in clusters, grids (e.g., Buhmann 1990), or otherwise provide much of the required freedom for good approximations.

The properties of conditional positive definiteness are fulfilled now for a much larger realm of radial basis functions which have still nowhere vanishing generalized Fourier transforms but with higher-order singularities at the origin. (Remember that this creates no problem for the well definedness of $\|\cdot\|_{\varphi}$.) For instance, the above properties are true for the thin-plate spline function $\varphi(r)=r^{2} \log r$, for the shifted logarithm $\varphi(r)=\left(r^{2}+c^{2}\right) \log \left(r^{2}+c^{2}\right)$, and for the multiquadric $\varphi(r)=-\sqrt{r^{2}+c^{2}}$. Here we still have a parameter $c$ which may now be arbitrary real. The order of the above is one for the multiquadric and two for the thinplate spline. Another commonly used radial basis function which gives rise to conditional positive definiteness is the $\varphi(r)=r^{3}$.

Hence the norm becomes a semi-norm with null-space $\mathbb{P}_{n}^{k}$, but it still has the same form as a square integral with the reciprocal of the Fourier transform of the radial basis function as a weight.

Therefore we have to include a polynomial from the null-space of the semi-norm to the approximant which becomes 
$s(x)=\sum_{\xi \in \Xi} \lambda_{\xi} \varphi(\|x-\xi\|)+q(x), \quad x \in \mathbb{R}^{n}$,

where $q \in \mathbb{P}_{n}^{k}$ and the side conditions on the coefficients

$$
\sum_{\xi \in \Xi} \lambda_{\xi} p(\xi)=0, \quad \forall p \in \mathbb{P}_{n}^{k}
$$

When quasi-interpolation is used, this inclusion of polynomials is not because they are not formed by interpolation condition, and the reproduction of polynomials (thus their presence in the linear space) is directly guaranteed by their construction.

If we consider the regularization network problem with the smoothing parameter $\mu$ again, then we have to solve the linear system with a smoothing parameter $\mu$ :

$$
(A+\mu I) \lambda+P^{T} b=f, \quad P \lambda=0,
$$

where $P=\left(p_{i}(\xi)\right)_{i=1, \ldots, L, \xi \in \Xi}$ and $p_{i}$ form a basis of $\mathbb{P}_{n}^{k}, b_{i}$ being the components of $b$, and $q(x)=\sum_{i=1}^{L} b_{i} p_{i}(x)$ is the expression of the polynomial added to the radial basis function sum. So in particular $P$ is a matrix with as many rows as the dimension $L=\left(\begin{array}{c}n+k \\ n\end{array}\right)$ of $\mathbb{P}_{n}^{k}$ is and $|\Xi|$ columns.

In all cases, the radial basis functions composed of the Euclidean norm can be regarded as reproducing kernels in the semi-Hilbert spaces defined by the set $X$ of distributions $f$ for which $\|g\|_{\varphi}$ is finite and the semi-inner product

$$
\begin{aligned}
(h, g)= & \frac{1}{(2 \pi)^{n}} \int_{\mathbb{R}^{n}} \frac{1}{\hat{\varphi}(\|x\|)} \hat{h}(x) \overline{\hat{g}(x)} d x, \\
& h, g, \in X
\end{aligned}
$$

In particular, $\|g\|_{\varphi}^{2}=(g, g)$. If the evaluation functional is continuous (bounded) on that space $X$, there exists a reproducing kernel, i.e., there is a $K: X \times X \rightarrow \mathbb{R}$ such that

$$
g(x)=(g, K(\cdot, x)), \quad \forall x \in \mathbb{R}^{n}, g \in X,
$$

See, for example, Wahba (1990). If the semiinner product is actually an inner product, then the reproducing kernel is unique. The kernel gives rise to positive definite matrices $\{K(\xi, \zeta)\}_{\xi, \zeta \in \Xi}$ if and only if it is a positive operator. For the spaces $X$ defined by our radial basis functions, it turns out that $K(x, y):=\varphi(\|x-y\|)$; see, e.g., the overview in Buhmann (2003). Then the matrices $A$ are positive definite if $\hat{\varphi}(\|\cdot\|)$ is well defined and positive, but if it has a singularity at zero, the $A$ may be only conditionally positive definite. Note here that $\hat{\varphi}(\|\cdot\|)$ denotes the $n$ variate Fourier transform of $\varphi(\|\cdot\|)$, both being radially symmetric.

\section{Advantages of the Approach}

Why are we interested in using radial basis functions for networks? The radial basis functions have many excellent approximation properties which make them useful as general tools for approximation. Among them are the variety of more or less smoothness as required (e.g., multiquadrics is $C^{\infty}$ for positive $c$ and just continuous for $c=0$ ), the fast evaluation and computation methods available (see, e.g., Beatson and Powell 1994), the aforementioned nonsingularity properties and their connection with the theory of reproducing kernel Hilbert spaces, and finally their excellent convergence properties (see, e.g., Buhmann 2003). Generally, neural networks are a tried and tested approach to approximation, modeling, and smoothing by methods from learning theory.

\section{Limitations}

The number of applications where the radial basis function approach has been used is vast. Also, the solutions may be computed efficiently by farfield expansions, approximated Lagrange functions, and multipole methods. However, there are still some limitations with these important computational methods when the dimension $n$ is large. So far, most of the multipole and far- 
field methods have been implemented only for medium-sized dimensions.

\section{Cross-References}

- Neural Networks

$\checkmark$ Regularization

\section{Recommended Reading}

Beatson RK, Powell MJD (1994) An iterative method for thin plate spline interpolation that employs approximations to Lagrange functions. In: Griffiths DF, Watson GA (eds) Numerical analysis 1993. Longman, Burnt Mill, pp 17-39

Broomhead D, Lowe D (1988) Radial basis functions, multi-variable functional interpolation and adaptive networks. Complex Syst 2:321-355

Buhmann MD (1990) Multivariate cardinalinterpolation with radial-basis functions. Construct Approx 6:225-255

Buhmann MD (1993) On quasi-interpolation with radial basis functions. J Approx Theory 72:103-130

Buhmann MD (1998) Radial functions on compact support. Proc Edinb Math Soc 41:33-46

Buhmann MD (2003) Radial basis functions: theory and implementations. Cambridge University Press, Cambridge

Buhmann MD, Porcu E, Daley D, Bevilacqua M (2013) Radial basis functions for multivariate geostatistics. Stoch Env Res Risk Assess 27(4): 909-922

Duchon J (1976) Interpolation des fonctions de deux variables suivant le principe de la flexion des plaques minces. RAIRO 10:5-12

Evgeniou T, Poggio T, Pontil M (2000) Regularization networks and support vector machines. Adv Comput Math 13:1-50

Hardy RL (1990) Theory and applications of the multiquadric-biharmonic method. Comput Math Appl 19:163-208

Micchelli CA (1986) Interpolation of scattered data: distance matrices and conditionally positive definite functions. Construct Approx 1:11-22

Pinkus A (1996) TDI-subpaces of $C\left(\mathbb{R}^{d}\right)$ and some density problems from neural networks. J Approx Theory 85:269-287

Schoenberg IJ (1938) Metric spaces and completely monotone functions. Ann Math 39:811-841

Tichonov AN, Arsenin VY (1977) Solution of Illposed problems. W.H. Winston, Washington, DC

Vapnik VN (1996) Statistical learning theory. Wiley, New York
Wahba G (1985) A comparison of GCV and GML for choosing the smoothing parameter in the generalized splines smoothing problem. Ann Stat 13:13781402

Wahba G (1990) Spline models for observational data. Series in applied mathematics, vol 59. SIAM, Philadelphia

\section{Radial Basis Function Neural Networks}

- Radial Basis Function Networks

\section{Random Decision Forests}

Random Forests

\section{Random Forests}

\section{Synonyms}

Random decision forests

\section{Definition}

Random Forests is an $\triangleright$ ensemble learning technique. It is a hybrid of the $>$ Bagging algorithm and the random subspace method, and uses - decision trees as the base classifier. Each tree is constructed from a bootstrap sample from the original dataset. An important point is that the trees are not subjected to pruning after construction, enabling them to be partially overfitted to their own sample of the data. To further diversify the classifiers, at each branch in the tree, the decision of which feature to split on is restricted to a random subset of size $n$, from the full feature set. The random subset is chosen anew for each branching point. $n$ is suggested to be $\log _{2}(N+1)$, where $N$ is the size of the whole feature set. 


\section{Random Subspace Method}

\section{Synonyms}

Random subspaces; RSM

\section{Definition}

The random subspace method is an rensemble learning technique. The principle is to increase diversity between members of the ensemble by restricting classifiers to work on different random subsets of the full feature space. Each classifier learns with a subset of size $n$, chosen uniformly at random from the full set of size $N$. Empirical studies have suggested good results can be obtained with the rule-of-thumb to choose $n=$ $N / 2$ features. The method is generally found to perform best when there are a large number of features (large $N$ ), and the discriminative information is spread across them. The method can underperform in the converse situation, when there are few informative features, and a large number of noisy/irrelevant features. - Random Forests is an algorithm combining RSM with the $>$ Bagging algorithm, which can provide significant gains over each used separately.

\section{Random Subspaces}

- Random Subspace Method

\section{Randomized Decision Rule}

- Markovian Decision Rule

\section{Randomized Experiments}

- Online Controlled Experiments and A/B Testing

\section{Rank Correlation}

Johannes Fürnkranz ${ }^{1,2}$ and Eyke Hüllermeier ${ }^{3}$

${ }^{1}$ Knowledge Engineering Group, TU Darmstadt, Darmstadt, Deutschland

${ }^{2}$ Department of Information Technology, University of Leoben, Leoben, Austria

${ }^{3}$ Department of Computer Science, Paderborn

University, Paderborn, Germany

\section{Abstract}

Rank correlation measures the correspondence between two rankings, $\tau$ and $\tau^{\prime}$, of a set of $m$ objects.

\section{Method}

Various proposals for such measures have been made, especially in the field of statistics. Two of the best-known measures are Spearman's rank correlation and Kendall's tau.

Spearman's rank correlation (Spearman 1904) calculates the sum of squared rank distances and is normalized such that it evaluates to -1 for reversed and to +1 for identical rankings. Formally, it is defined as follows:

$$
\left(\tau, \tau^{\prime}\right) \mapsto 1-\frac{6 \sum_{i=1}^{m}\left(\tau(i)-\tau^{\prime}(i)\right)^{2}}{m\left(m^{2}-1\right)}
$$

Kendall's tau (Kendall 1938) is the number of pairwise rank inversions between $\tau$ and $\tau^{\prime}$, again normalized to the range $[-1,+1]$ :

$$
\left(\tau, \tau^{\prime}\right) \mapsto 1
$$

$$
-\frac{4\left|\left\{(i, j) \mid i<j, \tau(i)<\tau(j) \wedge \tau^{\prime}(i)>\tau^{\prime}(j)\right\}\right|}{m(m-1)}
$$


Spearman's rank correlation and Kendall's tau give equal weight to all ranking positions, which is not desirable for all applications. For example, ranking problems in information retrieval are often evaluated with the (normalized) discounted cumulative gain (NDCG), which assigns more weight to the lower-ranking positions (cf. $>$ learning to rank).

\section{Cross-References}

Learning to Rank

$\checkmark$ Preference Learning

$\rightarrow$ ROC Analysis

\section{Recommended Reading}

Kendall M (1938) A new measure of rank correlation. Biometrika 30(1):81-89

Spearman C (1904) The proof and measurement of association between two things. Am J Psychol 15: 2-101

\section{Ratio Scale}

A ratio measurement scale possesses all the characteristics of interval measurement, and there exists a zero that, the same as arithmetic zero, means "nil" or "nothing." See > Measurement Scales.

\section{Real-Time Dynamic Programming}

Real-Time Dynamic Programming (RTDP) is the same as - Adaptive Real-Time Dynamic Programming (ARTDP) without the system identification component. It is applicable when an accurate model of the problem is available. It converges to an optimal policy of a stochastic optimal path problem under suitable conditions. RTDP was introduced by Barto et al. (1995) in their paper Learning to Act Using RTDP.

\section{Recall}

Recall is a measure of information retrieval performance. Recall is the total number of documents retrieved that are elevant/Total number of relevant documents in the database. See $\triangleright$ Precision and Recall.

\section{Cross-References}

Sensitivity

\section{Receiver Operating Characteristic Analysis}

- ROC Analysis

\section{Recognition}

Classification

\section{Recommender Systems}

Prem Melville and Vikas Sindhwani

IBM T. J. Watson Research Center, Yorktown Heights, NY, USA

\section{Definition}

The goal of a recommender system is to generate meaningful recommendations to a collection of users for items or products that might interest them. Suggestions for books on Amazon, or movies on Netflix, are real-world examples of the operation of industry-strength recommender systems. The design of such recommendation engines depends on the domain and the particular 
characteristics of the data available. For example, movie watchers on Netflix frequently provide ratings on a scale of 1 (disliked) to 5 (liked). Such a data source records the quality of interactions between users and items. Additionally, the system may have access to user-specific and itemspecific profile attributes such as demographics and product descriptions, respectively. Recommender systems differ in the way they analyze these data sources to develop notions of affinity between users and items, which can be used to identify well-matched pairs. $>$ Collaborative Filtering systems analyze historical interactions alone, while $\triangleright$ Content-based Filtering systems are based on profile attributes; and hybrid techniques attempt to combine both of these designs. The architecture of recommender systems and their evaluation on real-world problems is an active area of research.

\section{Motivation and Background}

Obtaining recommendations from trusted sources is a critical component of the natural process of human decision making. With burgeoning consumerism buo-yed by the emergence of the web, buyers are being presented with an increasing range of choices while sellers are being faced with the challenge of personalizing their advertising efforts. In parallel, it has become common for enterprises to collect large volumes of transactional data that allows for deeper analysis of how a customer base interacts with the space of product offerings. Recommender systems have evolved to fulfill the natural dual need of buyers and sellers by automating the generation of recommendations based on data analysis.

The term "collaborative filtering" was introduced in the context of the first commercial recommender system, called Tapestry (Goldberg et al. 1992), which was designed to recommend documents drawn from newsgroups to a collection of users. The motivation was to leverage social collaboration in order to prevent users from getting inundated by a large volume of streaming documents. Collaborative filtering, which analyzes usage data across users to find well-matched user-item pairs, has since been juxtaposed against the older methodology of content filtering, which had its original roots in information retrieval. In content filtering, recommendations are not "collaborative" in the sense that suggestions made to a user do not explicitly utilize information across the entire user-base. Some early successes of collaborative filtering on related domains included the GroupLens system (Resnick et al. 1994b).

As noted in Billsus and Pazzani (1998), initial formulations for recommender systems were based on straightforward correlation statistics and predictive modeling, not engaging the wider range of practices in statistics and machine learning literature. The collaborative filtering problem was mapped to classification, which allowed dimensionality reduction techniques to be brought into play to improve the quality of the solutions. Concurrently, several efforts attempted to combine content-based methods with collaborative filtering, and to incorporate additional domain knowledge in the architecture of recommender systems.

Further research was spurred by the public availability of datasets on the web, and the interest generated due to direct relevance to ecommerce. Netflix, an online streaming video and DVD rental service, released a large-scale dataset containing 100 million ratings given by about half-a-million users to thousands of movie titles, and announced an open competition for the best collaborative filtering algorithm in this domain. Matrix Factorization (Bell et al. 2009) techniques rooted in numerical linear algebra and statistical matrix analysis emerged as a state-ofthe-art technique.

Currently, recommender systems remain an active area of research, with a dedicated ACM conference, intersecting several subdisciplines of statistics, machine learning, data mining, and information retrievals. App-lications have been pursued in diverse domains ranging from recommending webpages to music, books, movies, and other consumer products. 


\section{Structure of Learning System}

The most general setting in which recommender systems are studied is presented in Fig. 1. Known user preferences are represented as a matrix of $n$ users and $m$ items, where each cell $r_{u, i}$ corresponds to the rating given to item $i$ by the user $u$. This user ratings matrix is typically sparse, as most users do not rate most items. The recommendation task is to predict what rating a user would give to a previously unrated item. Typically, ratings are predicted for all items that have not been observed by a user, and the highest rated items are presented as recommendations. The user under current consideration for recommendations is referred to as the active user.

The myriad approaches to recommender systems can be broadly categorized as:

- Collaborative Filtering ( $C F)$ : In CF systems, a user is recommended items based on the past ratings of all users collectively.

- Content-based recommending: These approaches recommend items that are similar in content to items the user has liked in the past, or matched to pre-defined attributes of the user.

- Hybrid approaches: These methods combine both collaborative and content-based approaches.

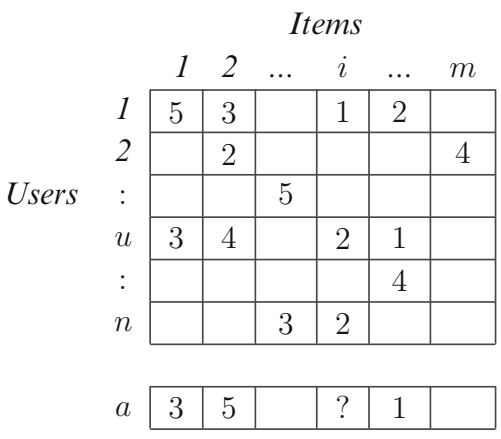

Recommender Systems, Fig. 1 User ratings matrix, where each cell $r_{u, i}$ corresponds to the rating of user $u$ for item $i$. The task is to predict the missing rating $r_{a, i}$ for the active user $a$

\section{Collaborative Filtering}

Collaborative filtering (CF) systems work by collecting user feedback in the form of ratings for items in a given domain and exploiting similarities in rat-ing behavior amongst several users in determining how to recommend an item. CF methods can be further subdivided into neighborhood-based and model-based approaches. Neighborhood-based methods are also commonly referred to as memory-based approaches (Breese et al. 1998).

\section{Neighborhood-Based Collaborative Filtering}

In neighborhood-based techniques, a subset of users are chosen based on their similarity to the active user, and a weighted combination of their ratings is used to produce predictions for this user. Most of these approaches can be generalized by the algorithm summarized in the following steps:

1. Assign a weight to all users with respect to similarity with the active user.

2. Select $k$ users that have the highest similarity with the active user - commonly called the neighborhood.

3. Compute a prediction from a weighted combination of the selected neighbors' ratings.

In step 1, the weight $w_{a, u}$ is a measure of similarity between the user $u$ and the active user $a$. The most commonly used measure of similarity is the Pearson correlation coefficient between the ratings of the two users (Resnick et al. 1994a), defined below:

$$
w_{a, u}=\frac{\sum_{i \in I}\left(r_{a, i}-\bar{r}_{a}\right)\left(r_{u, i}-\bar{r}_{u}\right)}{\sqrt{\sum_{i \in I}\left(r_{a, i}-\bar{r}_{a}\right)^{2} \sum_{i \in I}\left(r_{u, i}-\bar{r}_{u}\right)^{2}}}
$$

where $I$ is the set of items rated by both users, $r_{u, i}$ is the rating given to item $i$ by user $u$, and $\bar{r}_{u}$ is the mean rating given by user $u$.

In step 3, predictions are generally computed as the weighted average of deviations from the neighbor's mean, as in:

$$
p_{a, i}=\bar{r}_{a}+\frac{\sum_{u \in K}\left(r_{u, i}-\bar{r}_{u}\right) \times w_{a, u}}{\sum_{u \in K} w_{a, u}}
$$


where $p_{a, i}$ is the prediction for the active user $a$ for item $i, w_{a, u}$ is the similarity between users $a$ and $u$, and $K$ is the neighborhood or set of most similar users.

Similarity based on Pearson correlation measures the extent to which there is a linear dependence between two variables. Alternatively, one can treat the ratings of two users as a vector in an $m$-dimensional space, and compute similarity based on the cosine of the angle between them, given by:

$$
\begin{array}{r}
W_{a, u}=\cos \left(\mathbf{r}_{a}, \mathbf{r}_{u}\right)=\frac{\mathbf{r}_{a} \cdot \mathbf{r}_{u}}{\left\|\mathbf{r}_{a}\right\|_{2} \times\left\|r_{u}\right\|_{2}} \\
=\frac{\sum_{i=1}^{m} r_{a, i} r_{u, i}}{\sqrt{\sum_{i=1}^{m} r_{a, i}^{2}} \sqrt{\sum_{i=1}^{m} r_{u, i}^{2}}}
\end{array}
$$

When computing cosine similarity, one cannot have negative ratings, and unrated items are treated as having a rating of zero. Empirical studies (Breese et al. 1998) have found that Pearson correlation generally performs better. There have been several other similarity measures used in the literature, including Spearman rank correlation, Kendall's $\tau$ correlation, mean squared differences, entropy, and adjusted cosine similarity (Herlocker et al. 1999; Su and Khoshgoftaar 2009).

Several extensions to neighborhood-based CF, which have led to improved performance are discussed below.

Item-based Collaborative Filtering: When applied to millions of users and items, conventional neighborhood-based CF algorithms do not scale well, because of the computational complexity of the search for similar users. As a alternative, Linden et al. (2003) proposed item-to-item collaborative filtering where rather than matching similar users, they match a user's rated items to similar items. In practice, this approach leads to faster online systems, and often results in improved recommendations (Linden et al. 2003; Sarwar et al. 2001).

In this approach, similarities between pairs of items $i$ and $j$ are computed off-line using Pearson correlation, given by:

$$
w_{i, j}=\frac{\sum_{u \in U}\left(r_{u, i}-\bar{r}_{i}\right)\left(r_{u, j}-\bar{r}_{j}\right)}{\sqrt{\sum_{u \in U}\left(r_{u, i}-\bar{r}_{i}\right)^{2}} \sqrt{\sum_{u \in U}\left(r_{u, j}-\bar{r}_{j}\right)^{2}}}
$$

where $U$ is the set of all users who have rated both items $i$ and $j, r_{u, i}$ is the rating of user $u$ on item $i$, and $j, r_{u, i}$ is the average rating of the $i$ th item across users.

Now, the rating for item $i$ for user $a$ can be predicted using a simple weighted average, as in:

$$
p_{a, i}=\frac{\Sigma_{j \in K} r_{a, j} w_{i, j}}{\Sigma_{j \in K}\left|w_{i, j}\right|}
$$

where $K$ is the neighborhood set of the $k$ items rated by $a$ that are most similar to $i$.

For item-based collaborative filtering too, one may use alternative similarity metrics such as $a d-$ justed cosine similarity. A good empirical comparison of variations of item-based methods can be found in Sarwar et al. (2001).

Significance Weighting: It is common for the active user to have highly correlated neighbors that are based on very few co-rated (overlapping) items. These neighbors based on a small number of overlapping items tend to be bad predictors. One approach to tackle this problem is to multiply the similarity weight by a significance weighting factor, which devalues the correlations based on few co-rated items (Herlocker et al. 1999).

Default Voting: An alternative approach to dealing with correlations based on very few co-rated items is to assume a default value for the rating for items that have not been explicitly rated. In this way one can now compute correlation (Eq. 1) using the union of items rated by users being matched as opposed to the intersection. Such a default voting strategy has been shown to improve collaborative filtering by Breese et al. (1998).

Inverse User Frequency: When measuring the similarity between users, items that have been rated by all (and universally liked or disliked) are not as useful as less common items. To account for this Breese et al. (1998) introduced 
the notion of inverse user frequency, which is computed as $f_{i}=\log n / n_{i}$, where $n_{i}$ is the number of users who have rated item $i$ out of the total number of $n$ users. To apply inverse user frequency while using similarity-based $\mathrm{CF}$, the original rating is transformed for $i$ by multiplying it by the factor $f_{i}$. The underlying assumption of this approach is that items that are universally loved or hated are rated more frequently than others.

Case Amplification: In order to favor users with high similarity to the active user, Breese et al. (1998) introduced case amplification which transforms the original weights in Eq. (2) to

$$
w_{a, u}^{\prime}=w_{a, u} \cdot\left|w_{a, u}\right|^{\rho-1}
$$

where $\rho$ is the amplification factor, and $\rho \geq 1$.

Other notable extensions to similarity-based collaborative filtering include weighted majority prediction (Nakamura and Abe 1998) and imputation-boosted CF (Su et al. 2008).

Model-based Collaborative Filtering: Modelbased techniques provide recommendations by estimating parameters of statistical models for user ratings. For example, Billsus and Pazzani (1998) describe an early approach to map CF to a classification problem, and build a classifier for each active user representing items as features over users and available ratings as labels, possibly in conjunction with dimensionality reduction techniques to overcome data sparsity issues. Other predictive modeling techniques have also been applied in closely related ways.

More recently, $>$ latent factor and matrix factorization models have emerged as a state-of-theart methodology in this class of techniques (Bell et al. 2009). Unlike neighborhood based methods that generate recommendations based on statistical notions of similarity between users, or between items, latent factor models assume that the similarity between users and items is simultaneously induced by some hidden lower-dimensional structure in the data. For example, the rating that a user gives to a movie might be assumed to depend on few implicit factors such as the user's taste across various movie genres. Matrix factorization techniques are a class of widely successful latent factor models where users and items are simultaneously represented as unknown feature vectors (column vectors) $w_{u}, h_{i} \in \mathbb{R}^{k}$ along $k$ latent dimensions. These feature vectors are learnt so that inner products $w_{u}^{T} h_{i}$ approximate the known preference ratings $r_{u, i}$ with respect to some loss measure. The squared loss is a standard choice for the loss function, in which case the following objective function is minimized,

$$
j(W, H)=\sum_{(u, i) \in L}\left(r_{u, i}-w_{u}^{T} h_{i}\right)^{2}
$$

where $W=\left[w_{1} \ldots w_{n}\right]^{T}$ is an $n \times k$ matrix, $H=\left[h_{1} \ldots h_{m}\right]$ is a $k \times m$ matrix, and $L$ is the set of user-item pairs for which the ratings are known. In the impractical limit where all useritem ratings are known, the above objective function is $J(W, H)=\|R-W H\|_{\text {fro }}^{2}$ where $R$ denotes the $n \times m$ fully known user-item matrix. The solution to this problem is given by taking the truncated SVD of $R, R=U D V^{T}$ and setting $W=$ $U_{K} D_{k}^{\frac{1}{2}}, H=D_{k}^{\frac{1}{2}} V_{k}^{T}$ where $U_{k}, D_{k}, V_{k}$ contain the $k$ largest singular triplets of $R$. However, in the realistic setting where the majority of useritem ratings are unknown and insufficient number of matrix entries are observed, such a nice globally optimal solution cannot in general be directly obtained, and one has to explicitly optimize the non-convex objective function $J(W, H)$. Note that in this case, the objective function is a particular form of weighted loss, that is, $J(W, H)=$ $\|S \odot(R-W H)\|_{\text {fro }}^{2}$ where $\odot$ denotes elementwise products, and $S$ is a binary matrix that equals one over known user-item pairs $L$, and 0 otherwise. Therefore, weighted low-rank approximations are pertinent to this discussion (Srebro and Jaakkola 2003). Standard optimization procedures include gradient-based techniques, or procedures like alternating least squares where $H$ is solved keeping $W$ fixed and vice versa until a convergence criterion is satisfied. Note that fixing either $W$ or $H$ turns the problem of estimating the other into a weighted $>$ linear regression task. In order to avoid learning a model that overfits, it is 
common to minimize the objective function in the presence of $>$ regularization terms, $J(W, H)+$ $\gamma\|W\|^{2}+\lambda\|H\|^{2}$, where $\gamma, \lambda$ are regularization parameters that can be determined by crossvalidation. Once $W, H$ are learnt, the product $W H$ provides an approximate reconstruction of the rating matrix from where recommendations can be directly read off.

Different choices of loss functions, regularizers, and additional model constraints have generated a large body of literature on matrix factorization techniques. Arguably, for discrete ratings, the squared loss is not the most natural loss function. The maximum margin matrix factorization (Rennie and Srebro 2005) approach uses margin-based loss functions such as the hinge loss used in $>$ SVM classification, and its ordinal extensions for handling multiple ordered rating categories. For ratings that span over $K$ values, this reduces to finding $K-1$ thresholds that divide the real line into consecutive intervals specifying rating bins to which the output is mapped, with a penalty for insufficient margin of separation. Rennie and Srebro (2005) suggest a nonlinear conjugate gradient algorithm to minimize a smoothed version of this objective function.

Another class of techniques is the nonnegative matrix factorization popularized by the work of Lee and Seung (1999) where nonnegativity constraints are imposed on $W, H$. There are weighted extensions of NMF that can be applied to recommendation problems. The rating behavior of each user may be viewed as being a manifestation of different roles, for example, a composition of prototypical behavior in clusters of users bound by interests or community. Thus, the ratings of each user are an additive sum of basis vectors of ratings in the item space. By disallowing subtractive basis, nonnegativity constraints lend a part-based interpretation to the model. NMF can be solved with a variety of loss functions, but with the generalized KLdivergence loss defined as follows,

$$
J(W, H)=\sum_{u, i \in L} r_{u, i} \log \frac{r_{u, i}}{w_{u}^{T} h_{i}}-r_{u, i}+w_{u}^{T} h_{i}
$$

$\mathrm{NMF}$ is in fact essentially equivalent to probabilistic latent semantic analysis (pLSA) which has also previously been used for collaborative filtering tasks (Hofmann 2004).

The recently concluded million-dollar Netflix competition has catapulted matrix factorization techniques to the forefront of recommender technologies in collaborative filtering settings (Bell et al. 2009). While the final winning solution was a complex ensemble of different models, several enhancements to basic matrix factorization models were found to lead to improvements. These included:

1. The use of additional user-specific and itemspecific parameters to account for systematic biases in the ratings such as popular movies receiving higher ratings on average.

2. Incorporating temporal dynamics of rating behavior by introducing time-dependent variables.

In many settings, only implicit preferences are available, as opposed to explicit like-dislike ratings. For example, large business organizations, typically, meticulously record transactional details of products purchased by their clients. This is a one-class setting since the business domain knowledge for negative examples that a client has no interest in buying a product ever in the future is typically not available explicitly in corporate databases. Moreover, such knowledge is difficult to gather and maintain in the first place, given the rapidly changing business environment. Another example is recommending TV shows based on watching habits of users, where preferences are implicit in what the users chose to see without any source of explicit ratings. Recently, matrix factorization techniques have been advanced to handle such problems (Pan and Scholz 2009) by formulating confidence weighted objective function, $J(W, H)=\Sigma_{(u, i)} c_{u, i}\left(r_{u, i}-w_{u}^{T} h_{i}\right)^{2}$, under the assumption that unobserved user-item pairs may be taken as negative examples with a certain degree of confidence specified via $c_{u, i}$.

The problem of recovering missing values in a matrix from a small fraction of observed entries is also known as the Matrix Comple- 
tion problem. Recent work by Candès and Tao (2009) and Recht (2009) has shown that under certain assumptions on the singular vectors of the matrix, the matrix completion problem can be solved exactly by a convex optimization problem provided with a sufficient number of observed entries. This problem involves finding among all matrices consistent with the observed entries, the one with the minimum nuclear norm (sum of singular values).

\section{Content-Based Recommending}

Pure collaborative filtering recommenders only utilize the user ratings matrix, either directly, or to induce a collaborative model. These approaches treat all users and items as atomic units, where predictions are made without regard to the specifics of individual users or items. However, one can make a better personalized recommendation by knowing more about a user, such as demographic information (Pazzani 1999), or about an item, such as the director and genre of a movie (Melville et al. 2002). For instance, given movie genre information, and knowing that a user liked "Star Wars" and "Blade Runner," one may infer a predilection for science fiction and could hence recommend "Twelve Monkeys." Contentbased recommenders refer to such approaches, that provide recommendations by comparing representations of content describing an item to representations of content that interests the user. These approaches are sometimes also referred to as content-based filtering.

Much research in this area has focused on recommending items with associated textual content, such as web pages, books, and movies; where the web pages themselves or associated content like descriptions and user reviews are available. As such, several approaches have treated this problem as an information retrieval (IR) task, where the content associated with the user's preferences is treated as a query, and the unrated documents are scored with relevance/similarity to this query (Balabanovic and Shoham 1997). In NewsWeeder (Lang 1995), documents in each rating category are converted into $t f$-idf word vectors, and then averaged to get a prototype vector of each category for a user.
To classify a new document, it is compared with each prototype vector and given a predicted rating based on the cosine similarity to each category.

An alternative to IR approaches, is to treat recommending as a classification task, where each example represents the content of an item, and a user's past ratings are used as labels for these examples. In the domain of book recommending, Mooney and Roy (2000) use text from fields such as the title, author, synopses, reviews, and subject terms, to train a multinomial naive Bayes classifier. Ratings on a scale of 1 to $k$ can be directly mapped to $k$ classes (Melville et al. 2002), or alternatively, the numeric rating can be used to weight the training example in a probabilistic binary classification setting (Mooney and Roy 2000). Other classification algorithms have also been used for purely content-based recommending, including k-nearest neighbor, $\triangleright$ decision trees, and $\triangleright$ neural networks (Pazzani and Billsus 1997).

\section{Hybrid Approaches}

In order to leverage the strengths of content-based and collaborative recommenders, there have been several hybrid approaches proposed that combine the two. One simple approach is to allow both content-based and collaborative filtering methods to produce separate ranked lists of recommendations, and then merge their results to produce a final list (Cotter and Smyth 2000). Claypool et al. (1999) combine the two predictions using an adaptive weighted average, where the weight of the collaborative component increases as the number of users accessing an item increases.

Melville et al. (2002) proposed a general framework for content-boosted collaborative filtering, where content-based predictions are applied to convert a sparse user ratings matrix into a full ratings matrix, and then a CF method is used to provide recommendations. In particular, they use a Naïve Bayes classifier trained on documents describing the rated items of each user, and replace the unrated items by predictions from this classifier. They use the resulting pseudo ratings matrix to find neighbors similar to the active user, and produce predictions using Pearson correlation, appropriately weighted to 
account for the overlap of actually rated items, and for the active user's content predictions. This approach has been shown to perform better than pure collaborative filtering, pure contentbased systems, and a linear combination of the two. Within this content-boosted CF framework, $\mathrm{Su}$ et al. (2007) demonstrated improved results using a stronger content-predictor, TAN-ELR, and unweighted Pearson collaborative filtering.

Several other hybrid approaches are based on traditional collaborative filtering, but also maintain a content-based profile for each user. These content-based profiles, rather than co-rated items, are used to find similar users. In Pazzani's approach (Pazzani 1999), each user-profile is represented by a vector of weighted words derived from positive training examples using the Winnow algorithm. Predictions are made by applying $\mathrm{CF}$ directly to the matrix of user-profiles (as opposed to the user-ratings matrix). An alternative approach, Fab (Balabanovic and Shoham 1997), uses $>$ relevance feedback to simultaneously mold a personal filter along with a communal "topic" filter. Documents are initially ranked by the topic filter and then sent to a user's personal filter. The user's relevance feedback is used to modify both the personal filter and the originating topic filter. Good et al. (1999) use collaborative filtering along with a number of personalized information filtering agents. Predictions for a user are made by applying CF on the set of other users and the active user's personalized agents.

Several hybrid approaches treat recommending as a classification task, and incorporate collaborative elements in this task. Basu et al. (1998) use Ripper, a $>$ rule induction system, to learn a function that takes a user and movie and predicts whether the movie will be liked or disliked. They combine collaborative and content information, by creating features such as comedies liked by user and users who liked movies of genre X. In other work, Soboroff and Nicholas (1999) multiply a term-document matrix representing all item content with the user-ratings matrix to produce a content-profile matrix. Using latent semantic Indexing, a rank- $k$ approximation of the content-profile matrix is computed. Term vectors of the user's relevant documents are averaged to produce a user's profile. Then, new documents are ranked against each user's profile in the LSI space. Some hybrid approaches attempt to directly combine content and collaborative data under a single probabilistic framework. Popescul et al. (2001) extended Hofmann's aspect model (Hofmann 1999) to incorporate a three-way cooccurrence data among users, items, and item content. Their generative model assumes that users select latent topics, and documents and their content words are generated from these topics. Schein et al. (2002) extend this approach, and focus on making recommendations for items that have not been rated by any user.

\section{Evaluation Metrics}

The quality of a recommender system can be evaluated by comparing recommendations to a test set of known user ratings. These systems are typical measured using predictive accuracy metrics (Herlocker et al. 2004), where the predicted ratings are directly compared to actual user ratings. The most commonly used metric in the literature is $>$ Mean Absolute Error (MAE) - defined as the average absolute difference between predicted ratings and actual ratings, given by:

$$
\text { MAE }=\frac{\sum_{\{u, i\}}\left|P_{u, i}-r_{u, i}\right|}{N}
$$

Where $p_{u, i}$ is the predicted rating for user $u$ on item $i, r_{u, i}$ is the actual rating, and $N$ is the total number of ratings in the test set.

A related commonly used metric, Root Mean Squared Error (RMSE), puts more emphasis on larger absolute errors, and is given by:

$$
\mathrm{RMSE}=\sqrt{\frac{\sum_{\{u, i\}}\left(p_{u, i}-r_{u, i}\right)^{2}}{N}}
$$

Predictive accuracy metrics treat all items equally. However, for most recommender systems the primary concern is accurately predict the items a user will like. As such, researchers often view recommending as predicting good, that is, items with high ratings versus bad or poorly rated items. In the context of information retrieval (IR), identifying the 
good from the background of bad items can be viewed as discriminating between "relevant" and "irrelevant" items; and as such, standard IR measures, like $>$ Precision, $>$ Recall and - Area Under the ROC Curve (AUC) can be utilized. These, and several other measures, such as F1-measure, Pearson's product-moment correlation, Kendall's $\tau$, mean average precision, half-life utility, and normalized distance-based performance measure are discussed in more detail by Herlocker et al. (2004).

\section{Challenges and Limitations}

This section, presents some of the common hurdles in deploying recommender systems, as well as some research directions that address them.

Sparsity: Stated simply, most users do not rate most items and, hence, the user ratings matrix is typically very sparse. This is a problem for collaborative filtering systems, since it decreases the probability of finding a set of users with similar ratings. This problem often occurs when a system has a very high item-to-user ratio, or the system is in the initial stages of use. This issue can be mitigated by using additional domain information (Melville et al. 2002; Su et al. 2007) or making assumptions about the data generation process that allows for high-quality imputation (Su et al. 2008).

The Cold-Start Problem: New items and new users pose a significant challenge to recommender systems. Collectively these problems are referred to as the cold-start problem (Schein et al. 2002). The first of these problems arises in collaborative filtering systems, where an item cannot be recommended unless some user has rated it before. This issue applies not only to new items, but also to obscure items, which is particularly detrimental to users with eclectic tastes. As such the new-item problem is also often referred to as the first-rater problem. Since content-based approaches (Mooney and Roy 2000; Pazzani and Billsus 1997) do not rely on ratings from other users, they can be used to produce recommendations for all items, provided attributes of the items are available. In fact, the content-based predictions of similar users can also be used to further improve predictions for the active user (Melville et al. 2002).

The new-user problem is difficult to tackle, since without previous preferences of a user it is not possible to find similar users or to build a content-based profile. As such, research in this area has primarily focused on effectively selecting items to be rated by a user so as to rapidly improve recommendation performance with the least user feedback. In this setting, classical techniques from $>$ active learning can be leveraged to address the task of item selection (Harpale and Yang 2008; Jin and Si 2004).

Fraud: As recommender systems are being increasingly adopted by commercial websites, they have started to play a significant role in affecting the profitability of sellers. This has led to many unscrupulous vendors engaging in different forms of fraud to game recommender systems for their benefit. Typically, they attempt to inflate the perceived desirability of their own products (push attacks) or lower the ratings of their competitors (nuke attacks). These types of attack have been broadly studied as shilling attacks (Lam and Riedl 2004) or profile injection attacks (Burke et al. 2005). Such attacks usually involve setting up dummy profiles, and assume different amounts of knowledge about the system. For instance, the average attack (Lam and Riedl 2004) assumes knowledge of the average rating for each item; and the attacker assigns values randomly distributed around this average, along with a high rating for the item being pushed. Studies have shown that such attacks can be quite detrimental to predicted ratings, though itembased collaborative filtering tends to be more robust to these attacks (Lam and Riedl 2004). Obviously, content-based methods, which only rely on a users past ratings, are unaffected by profile injection attacks.

While pure content-based methods avoid some of the pitfalls discussed above, collaborative filtering still has some key advantages over them. Firstly, CF can perform in domains where there is not much content associated with items, or where the content is difficult for a computer to 
analyze, such as ideas, opinions, etc. Secondly, a $\mathrm{CF}$ system has the ability to provide serendipitous recommendations, that is, it can recommend items that are relevant to the user, but do not contain content from the user's profile.

\section{Recommended Reading}

Good surveys of the literature in the field can be found in Adomavicius and Tuzhilin (2005), $\mathrm{Su}$ (2009), Bell et al. (2009). For extensive empirical comparisons on variations of Collaborative Filtering refer to Breese et al. (1998), Herlocker et al. (1999), and Sarwar et al. (2001).

Adomavicius G, Tuzhilin A (2005) Toward the next generation of recommender systems: a survey of the state-of-the-art and possible extensions. IEEE Trans Knowl Data Eng 17(6):734-749

Balabanovic M, Shoham Y (1997) Fab: contentbased, collaborative recommendation. Commun Assoc Comput Mach 40(3):66-72

Basu C, Hirsh H, Cohen W (July 1998) Recommendation as classification: using social and content-based information in recommendation. In: Proceedings of the fifteenth national conference on artificial intelligence (AAAI-98), Madison, pp 714-720

Bell R, Koren Y, Volinsky C (2009) Matrix factorization techniques for recommender systems. IEEE Comput 42(8):30-37

Billsus D, Pazzani MJ (1998) Learning collaborative information filters. In: Proceedings of the fifteenth international conference on machine learning (ICML-98), Madison. Morgan Kaufmann, San Francisco, pp 46-54

Breese JS, Heckerman D, Kadie C (1998) Empirical analysis of predictive algorithms for collaborative filtering. In: Proceedings of the fourteenth conference on uncertainty in artificial intelligence, Madison

Burke R, Mobasher B, Bhaumik R, Williams C (2005) Segment-based injection attacks against collaborative filtering recommender systems. In: ICDM '05: proceedings of the fifth IEEE international conference on data mining, Houston. IEEE Computer Society, Washington, DC, pp 577-580

Candès EJ, Tao T (2009) The power of convex relaxation: near-optimal matrix completion. IEEE Trans Inf Theory 56(5):2053-2080

Claypool M, Gokhale A, Miranda T (1999) Combining content-based and collaborative filters in an online newspaper. In: Proceedings of the SIGIR-99 workshop on recommender systems: algorithms and evaluation, Berkeley

Cotter P, Smyth B (2000) PTV: intelligent personalized TV guides. In: Twelfth conference on inno- vative applications of artificial intelligence, Austin, pp 957-964

Goldberg D, Nichols D, Oki B, Terry D (1992). Using collaborative filtering to weave an information tapestry. Commun Assoc Comput Mach 35(12): 61-70

Good N, Schafer JB, Konstan JA, Borchers A, Sarwar B, Herlocker J et al (1999) Combining collaborative filtering with personal agents for better recommendations. In: Proceedings of the sixteenth national conference on artificial intelligence (AAAI-99), Orlando, pp 439-446

Harpale AS, Yang Y (2008) Personalized active learning for collaborative filtering. In: SIGIR '08: proceedings of the 31st annual international ACM SIGIR conference on research and development in information retrieval, Singapore. ACM, New York, pp 91-98

Herlocker J, Konstan J, Borchers A, Riedl J (1999) An algorithmic framework for performing collaborative filtering. In: Proceedings of 22nd international ACM SIGIR conference on research and development in information retrieval, Berkeley. ACM, New York, pp 230-237

Herlocker JL, Konstan JA, Terveen LG, Riedl JT (2004) Evaluating collaborative filtering recommender systems. ACM Trans Inf Syst 22(1): 5-53

Hofmann T (1999) Probabilistic latent semantic analysis. In: Proceedings of the fifteenth conference on uncertainty in artificial intelligence, Stockholm, 30 July-1 Aug 1999. Morgan Kaufmann

Hofmann T (2004) Latent semantic analysis for collaborative filtering. ACM Trans Inf Syst 22(1): 89-115

Jin R, Si L (2004) A Bayesian approach toward active learning for collaborative filtering. In: UAI '04: proceedings of the 20th conference on uncertainty in artificial intelligence, Banff. AUAI Press, Arlington, pp 278-285

Lam SK, Riedl J (2004) Shilling recommender systems for fun and profit. In: WWW '04: proceedings of the 13th international conference on World Wide Web, New York. ACM, New York, pp 393-402

Lang K (1995) NewsWeeder: learning to filter netnews. In: Proceedings of the twelfth international conference on machine learning (ICML-95), Tahoe City. Morgan Kaufmann, San Francisco, pp 331339. ISBN 1-55860-377-8.

Lee DD, Seung HS (1999) Learning the parts of objects by non-negative matrix factorization. Nature 401:788

Linden G, Smith B, York J (2003) Amazon.com recommendations: item-to-item collaborative filtering. IEEE Internet Comput 7(1):76-80

Melville P, Mooney RJ, Nagarajan R (2002) Contentboosted collaborative filtering for improved recommendations. In: Proceedings of the eighteenth national conference on artificial intelligence (AAAI02), Edmonton, pp 187-192 
Mooney RJ, Roy L (2000) Content-based book recommending using learning for text categorization. In: Proceedings of the fifth ACM conference on digital libraries, San Antonio, pp 195-204

Nakamura A, Abe N (1998) Collaborative filtering using weighted majority prediction algorithms. In: ICML '98: proceedings of the fifteenth international conference on machine learning, Madison. Morgan Kaufmann, San Francisco, pp 395-403

Pan R, Scholz M (2009) Mind the gaps: weighting the unknown in large-scale one-class collaborative filtering. In: 15th ACM SIGKDD conference on knowledge discovery and data mining (KDD), Paris

Pazzani MJ (1999) A framework for collaborative, content-based and demographic filtering. Artif Intell Rev 13(5-6):393-408

Pazzani MJ, Billsus D (1997) Learning and revising user profiles: the identification of interesting web sites. Mach Learn 27(3):313-331

Popescul A, Ungar L, Pennock DM, Lawrence S (2001) Probabilistic models for unified collaborative and content-based recommendation in sparse-data environments. In: Proceedings of the seventeenth conference on uncertainity in artificial intelligence. University of Washington, Seattle

Recht B (2009, to appear) A simpler approach to matrix completion. J Mach Learn Res

Rennie J, Srebro N (2005) Fast maximum margin matrix factorization for collaborative prediction. In: International conference on machine learning, Bonn

Resnick P, Iacovou N, Sushak M, Bergstrom P, Reidl J (1994a) GroupLens: an open architecture for collaborative filtering of netnews. In: Proceedings of the 1994 computer supported cooperative work conference, New York. ACM, New York

Resnick P, Neophytos I, Bergstrom P, Mitesh S, Riedl J (1994b) Grouplens: an open architecture for collaborative filtering of netnews. In: CSCW94 - conference on computer supported cooperative work, Chapel Hill. Addison-Wesley, pp 175-186

Sarwar B, Karypis G, Konstan J, Reidl J (2001) Itembased collaborative filtering recommendation algorithms. In: WWW '01: proceedings of the tenth international conference on World Wide Web, Hong Kong. ACM, New York, pp 285-295

Schein AI, Popescul A, Ungar LH, Pennock DM (2002) Methods and metrics for cold-start recommendations. In: SIGIR '02: proceedings of the 25th annual international ACM SIGIR conference on research and development in information retrieval, Tampere. ACM, New York, pp 253-260

Soboroff I, Nicholas C (1999) Combining content and collaboration in text filtering. In: Joachims $\mathrm{T}$ (ed) Proceedings of the IJCAI'99 workshop on machine learning in information filtering, Stockholm, pp 86-91

Srebro N, Jaakkola T (2003) Weighted low-rank approximations. In: International conference on machine learning (ICML), Washington, DC
Su X, Khoshgoftaar TM (2009) A survey of collaborative filtering techniques. Adv Artif Intell 2009: $1-20$

Su X, Greiner R, Khoshgoftaar TM, Zhu X (2007) Hybrid collaborative filtering algorithms using a mixture of experts. In: Web intelligence, pp 645-649

Su X, Khoshgoftaar TM, Zhu X, Greiner R (2008) Imputation-boosted collaborative filtering using machine learning classifiers. In: SAC '08: proceedings of the 2008 ACM symposium on applied computing. ACM, New York, pp 949-950

\section{Record Linkage}

Peter Christen $^{1}$ and William E. Winkler ${ }^{2}$

${ }^{1}$ Research School of Computer Science, The

Australian National University, Canberra, ACT,

Australia

${ }^{2}$ US Census Bureau, Suitland, MD, USA

\section{Abstract}

Many data mining and machine learning projects require information from various data sources to be integrated and linked before they can be used for further analysis. A crucial task of such data integration is to identify which records refer to the same real-world entities across databases when no common entity identifiers are available and when records can contain errors and variations. This process of record linkage therefore has to rely upon the attributes that are available in the databases to be linked. For databases that contain personal information, for example, of customers, taxpayers, or patients, these are commonly their names, addresses, phone numbers, and dates of birth.

To improve the scalability of the linkage process, blocking or indexing techniques are commonly applied to limit the comparison of records to pairs or groups that likely correspond to the same entity. Records are compared using a variety of comparison functions, most commonly approximate string comparators that account for typographical errors and variations in textual attributes. 
The compared records are then classified into matches, non-matches, and potential matches, depending upon the decision model used. If training data in the form of true matches and non-matches are available, supervised classification techniques can be employed. However, in many practical record linkage applications, no ground truth data are available, and therefore unsupervised approaches are required. An approach known as probabilistic record linkage is commonly employed. In this article we provide an overview of record linkage with an emphasis on the classification aspects of this process.

\section{Synonyms}

Authority control; Citation or reference matching (when applied to bibliographic data); Co-reference resolution; Data linkage; Data matching; Data reconciliation; Deduplication or duplicate detection (when applied to one database only); Entity resolution; Field scrubbing; Identity uncertainty; List washing; Merge-purge; Object identification; Object matching; Reference reconciliation

\section{Definition}

Identifying and linking records that correspond to the same real-world entity in one or more databases is an increasingly important task in many data mining and machine learning projects. The aim of record linkage is to compare records within one (known as deduplication) or across two databases and classify the compared pairs of records as matches (pairs where both records are assumed to refer to the same real-world entity) and non-matches (pairs where the two records are assumed to refer to different entities).

Formally, let us consider two databases (or files), $\mathbf{A}$ and $\mathbf{B}$, and record pairs in the product space $\mathbf{A} \times \mathbf{B}$ (for the deduplication of a single database $\mathbf{A}$, the product space is $\mathbf{A} \times \mathbf{A}$ ). The aim of record linkage is to classify these record pairs into the classes of matches (links) and non- matches (non-links) (Christen 2012). Depending upon the decision model used (Fellegi and Sunter 1969; Herzog et al. 2007), a third class of potential matches (potential links) might be used. These are difficult to classify record pairs that will need to be manually assessed and classified as matches or non-matches in a manual clerical review process.

Each record pair in $\mathbf{A} \times \mathbf{B}$ is assumed to correspond to either a true match or a true nonmatch. The space $\mathbf{A} \times \mathbf{B}$ is therefore partitioned into the set $M$ of true matches and the set $U$ of true non-matches. The objective of record linkage is to correctly classify record pairs from $M$ into the class of matches and pairs from $U$ into the class of non-matches.

\section{Motivation and Background}

Increasingly, information systems and data mining projects require data from multiple sources to be integrated and linked in order to improve data quality, enrich existing data sources, or facilitate data analysis that is not feasible on an individual database. Compared to analyzing databases in isolation, the analysis of data linked across disparate sources, either within a single or between different organizations, can lead to much improved benefits. Integrated data can also allow types of analyses that are not feasible on individual databases, for example, the detection of fraud or terrorism suspects through the analysis of certain suspicious patterns of activities or the identification of adverse drug reactions in particular patient groups (Christen 2012). Record linkage has been employed in a wide range of domains as we discuss in section "Applications" below.

In most cases the databases to be linked (or deduplicated) do not contain unique entity identifiers or keys. Therefore, attributes (fields) that are common across the databases need to be used to identify similar records that likely correspond to the same entity. If the databases contain information about people, then these common attributes can be names, addresses, dates of birth, and other partially identifying personal details. 
However, often the quality of such information is low, as personal details can be entered or recorded wrongly, be incomplete, or be out of date. Record linkage based on "dirty data" is challenging as ambiguities, errors, variations, and value changes can lead to both false matches (record pairs wrongly classified as referring to the same entity) and false non-matches (missed true matching pairs of records classified as nonmatches).

The term record linkage was used in 1946 by Halbert Dunn to describe the idea of assembling a book of life for all individuals in the world (Dunn 1946). Each such book would begin with a birth record and end with a death record and in between would contain marriage and divorce records, as well as records about a person's contacts with the health and social security systems. Dunn realized that having such books of life for a full population would provide a wealth of information that would allow governments to improve national statistics, better plan services, and also help to identify individuals.

The first computer-based linkage techniques were proposed in the 1950s and early 1960s by Howard Newcombe et al. (1959), who also developed the basic ideas of the successful probabilistic record linkage approach described in the following section. Based on Newcombe's ideas, Fellegi and Sunter in 1969 published their sem- inal paper on a theory for probabilistic record linkage (Fellegi and Sunter 1969). They proved that an optimal probabilistic decision rule can be found under the assumption of independence of the attributes used in the comparison of records. This influential work has been the basis for many record linkage systems and software products, and it is still widely used today.

\section{Theory/Solution}

We now describe the steps involved in the record linkage process, followed by a more detailed discussion of techniques used to classify record pairs into matches and non-matches. Note that for the deduplication of a single database, all steps of the record linkage process are still applicable.

\section{The Record Linkage Process}

Figure 1 provides an overview of the steps involved in the general record linkage process. We assume two databases that contain details about the same types of entities (such as people, businesses, scientific publications, and so on). The first step of data cleaning and standardization is important to convert the input data into the same format so they are more suitable for comparison. This step involves, for example, converting all letters into lower or upper case, removing

\section{Record Linkage, Fig. 1}

The general process of linking two databases (As adapted from Christen 2012)

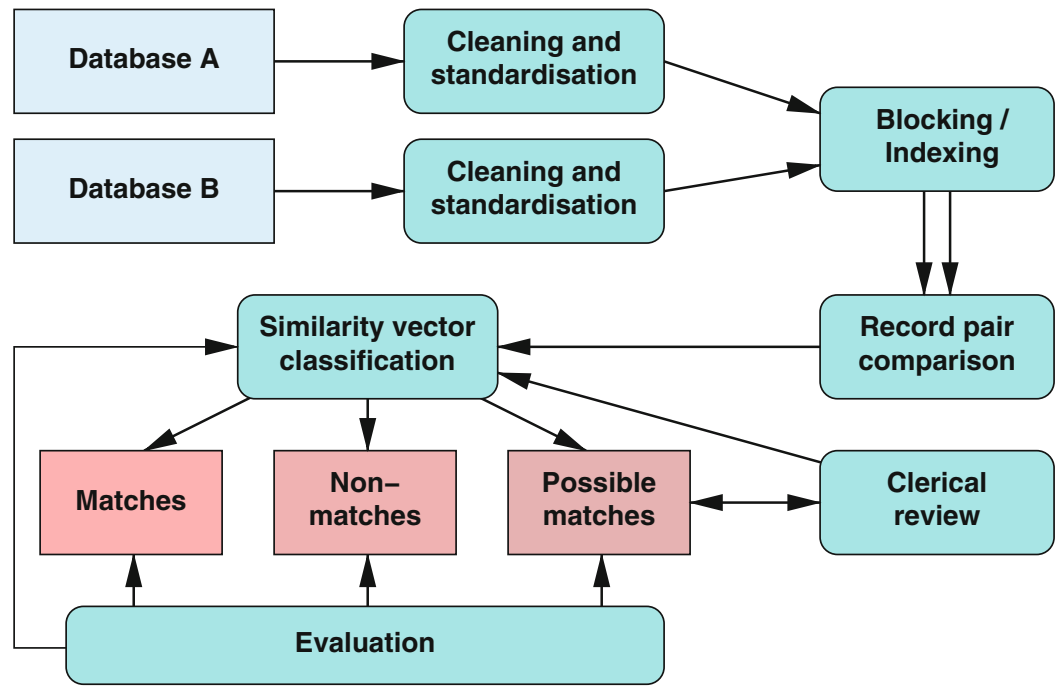


certain punctuations and words, and splitting attributes into specific fields (such as title, first name, middle name, and last name for personal names).

The second step of blocking or indexing is aimed at reducing the number of record pairs that need to be compared from the full pairwise comparison space of $|\mathbf{A}| \times|\mathbf{B}|$, where $|\cdot|$ is the number of records in a database. The idea is to only compare records in detail that likely refer to matches. This is accomplished by splitting the databases into blocks according to some criteria and then only comparing records in the same block across the two databases. An example criteria can, for example, be a postor zip code attribute, resulting in only records being compared that have the same post- or zip code value. Various such blocking or indexing techniques have been developed in the past few decades (Christen 2012).

In the comparison step, candidate record pairs generated in the blocking/indexing step are then compared in detail using a variety of attribute and record comparison functions. As many attributes used in record linkage to compare records contain textual values (like names and addresses), approximate string comparison functions such as Jaro-Winkler or edit distance are commonly used (Christen 2012). Specific comparison functions have also been developed for values such as ages, dates, or phone numbers (Christen 2012). Generally all these comparison functions return a numerical similarity, $s$, that is normalized in $0 \leq$ $s \leq 1$, with $s=1$ if two attribute values are the same (like "geoff" and "geoff"), $s=0$ if they are completely different (like "claude" and "geoff"), and $0<s<1$ if they are somehow similar (like "geoff" and "jeff"). For each compared record pair, a similarity vector (also known as weight vector) is formed that contains the similarities of all compared attributes of that pair.

In the classification step (as we discuss in more details below), each compared candidate record pair is classified into one of the classes of matches, non-matches, and possibly also potential matches, depending upon the decision model used (Fellegi and Sunter 1969; Herzog et al. 2007). Various classification techniques (both su- pervised and unsupervised) have been developed in the past nearly five decades.

If candidate record pairs have been classified into potential matches, a manual clerical review process is required to decide their final match status (match or non-match). These manual classifications can flow back into the classification model when an active learning approach is employed. Several active learning approaches have been developed for record linkage (Christen 2012).

In the final evaluation step, the complexity, completeness, and quality of the linked records are evaluated using a variety of measures (Christen 2012). The complexity of a linkage can be measured by the number of candidate record pairs generated by an indexing or blocking technique. Measuring completeness and linkage quality requires truth data in the form of known true matching and non-matching record pairs. Linkage quality is generally measured using precision and recall, while completeness is similar to recall but measures how many of all known true matches are included in the set of candidate record pairs (i.e., how many true matches are not removed in the indexing/blocking step).

\section{Record Linkage Model of Fellegi and Sunter}

Fellegi and Sunter (1969) provided a formal mathematical model for ideas that had been introduced by Newcombe et al. (1959). Fellegi and Sunter (1969) also provided ways of estimating key parameters without training data. Generally, training data have not been available for most record linkage applications.

Following the notation used in section "Definition" above, Fellegi and Sunter, making rigorous concepts introduced by Newcombe et al. (1959), considered ratios of probabilities of the form:

$$
R=\frac{P(\gamma \in \Gamma \mid M)}{P(\gamma \in \Gamma \mid U)}
$$

where $\gamma$ is an arbitrary agreement pattern in a comparison space $\Gamma$. For instance, $\Gamma$ might consist of eight patterns representing simple agreement or not on the largest name component, street 
name, and street number (Herzog et al. 2007). Alternatively, each $\gamma \in \Gamma$ might additionally account for the relative frequency with which specific values of name components such as "Smith", "Zabrinsky", "AAA", and "Capitol" occur. The ratio $R$ or any monotonically increasing function of it (such as the natural $\log$ ) is referred to as a matching weight (or score). The decision rule is given by:

If $R \geq T_{\mu}$, then designate pair as a match.

If $T_{\lambda}<R<T_{\mu}$, then designate pair as a possible match

and hold for clerical review.

If $R \leq T_{\lambda}$, then designate pair as a non-match.

The cutoff thresholds $T_{\lambda}$ and $T_{\mu}$ are determined by a priori error bounds on false matches and false non-matches. The thresholds are often called lower and upper cutoffs. Rule (2) agrees with intuition. If $\gamma \in \Gamma$ consists primarily of agreements, then it is intuitive that $\gamma \in \Gamma$ would be more likely to occur among matches than nonmatches, and the ratio (1) would be large. On the other hand, if $\gamma \in \Gamma$ consists primarily of disagreements, then the ratio (1) would be small. Rule (2) partitions the set $\gamma \in \Gamma$ into three disjoint subregions. The region $\left\{T_{\lambda}<R<T_{\mu}\right\}$ is referred to as the no-decision region or clerical review region (Herzog et al. 2007). In some situations, resources are available to review pairs clerically.

Figure 2 provides an illustration of the curves of $\log$ frequency versus log weight for matches and non-matches, respectively. The data used in Fig. 2 are based on information obtained while matching name and address files from one of the sites for the 1988 US Dress Rehearsal Census. The clerical review region consists of individuals within the same household that are missing both name and age. Figure 2 shows hypothetical cutoff thresholds that we denote with the symbols $L$ (lower) and $U$ (upper) in this figure, respectively.

\section{Learning Parameters via the Methods of Fellegi and Sunter}

Fellegi and Sunter (1969) were the first to provide very general methods for computing the probabilities in the ratio (1). As the methods are useful, we describe what they introduced and then show how the ideas led into more general methods that can be used for unsupervised learning (i.e., without training data) in a large number of situations.

Fellegi and Sunter observed several things. Firstly,

$$
P(S)=P(S \mid M) P(M)+P(S \mid U) P(U)
$$

for any set $S$ of pairs in $\mathbf{A} \times \mathbf{B}$. The probability on the left can be computed directly from the set of pairs. In Equation (3), $M$ and $U$ are restricted to $S$. Secondly, if sets $A^{x}$ represent simple agreement/disagreement, under the conditional independence assumption (CI) (i.e., naive Bayes), we obtain

$$
\begin{aligned}
& P\left(A_{1}^{x} \cap A_{2}^{x} \cap A_{3}^{x} \mid D\right) \\
& \quad=P\left(A_{1}^{x} \mid D\right) P\left(A_{2}^{x} \mid D\right) P\left(A_{3}^{x} \mid D\right) .
\end{aligned}
$$

Here $D$ is either $M$ or $U$. Then (3) and (4) provide seven equations and seven unknowns (as $x$ represents agree or disagree) that yield quadratic equations that they solved. Equation (or set of equations) (4) can be expanded to $K$ fields.

The expectation-maximization (EM) algorithm (Dempster et al. 1977) can be used to estimate the probabilities in Eqs. (3) and (4) when there are more than $K$ fields or when condition (CI) may not hold.

For the 1990 US Decennial Census, Winkler (1988) introduced an EM algorithm that found the best naive Bayes approximation of a general Bayes net model where interactions between fields were accounted for. This type of EM was necessary because "optimal" parameters were used for each of the $\sim 500$ regions into which the 
Record Linkage, Fig. 2

Log frequency versus weight, matches and non-matches combined

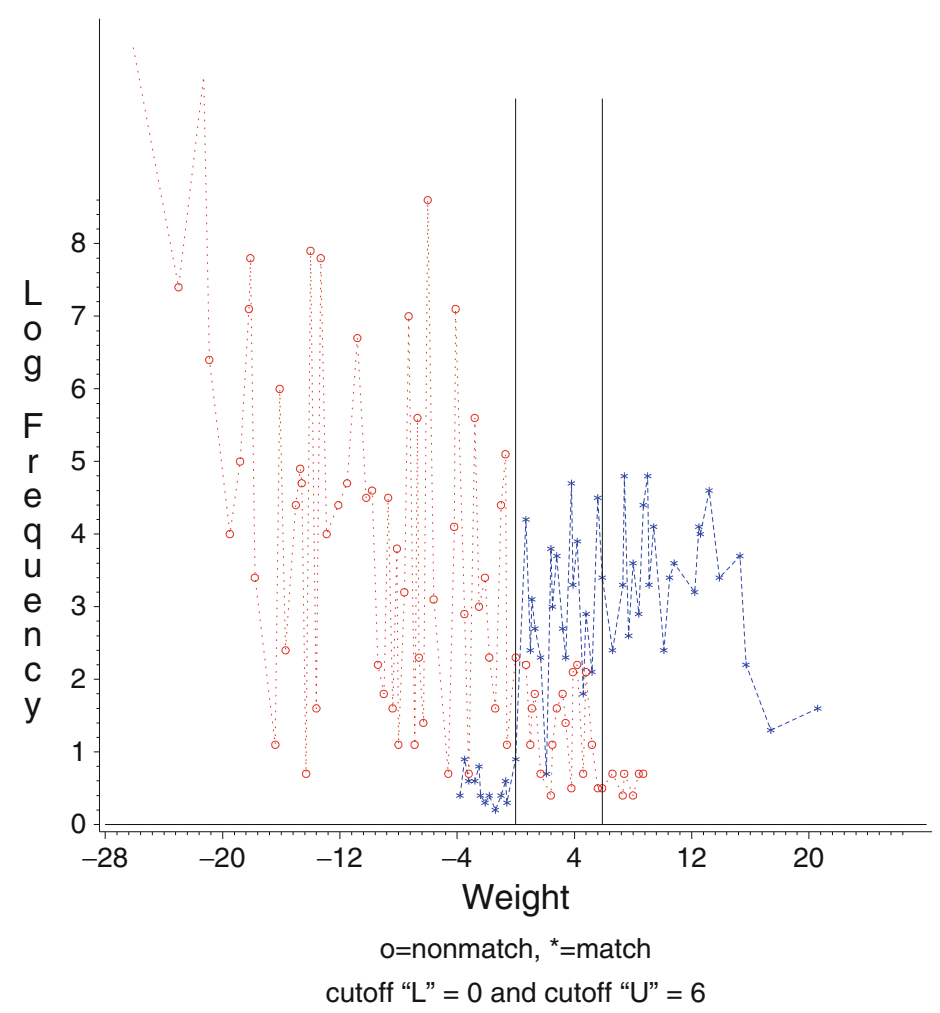

USA was divided, the entire matching operations needed to be completed in less than 6 weeks to provide estimates required under the US law, and it was impossible to obtain training data.

Herzog et al. (2010) provide many of the details of the EM procedures used in the 1990 US Decennial Census production matching systems that we do not cover here. We provide two highlights that were in Herzog et al. (2010). Firstly, the EM algorithm in this particular application was able to adapt automatically to increasing missing data. During 1 week in one of seven processing offices, it was discovered that the clerical review region increased significantly. Upon follow-up, it was determined that two clerks had managed to bypass keypunch edits on the yearof-birth field, and all records keyed by them disagreed on the computed age. Age and first name were the only fields that would allow distinguishing true matches within households.

Secondly, the probabilities from the unsupervised learning yielded better matching results than results from an iterative refinement procedure (a type of active learning) that was in widespread use for matching. In the iterative refinement procedure, a subset of clerical pairs were followed up to determine matches and nonmatches, the matching probabilities were reestimated, an additional set of clerical pairs were followed up, and parameters were reestimated, with the entire interactive learning procedure being repeated on the order of five cycles until the matching probabilities stabilized.

Superficially, the EM algorithm (Winkler 1988) considers different orderings of the form

$$
\begin{aligned}
& P\left(A_{\rho, 1}^{x} \cap \cdots \cap A_{\rho, k}^{x} \mid D\right) \\
& \quad=\prod_{i=1}^{K} P\left(A_{\rho, i}^{x} \mid A_{\rho, i-1}^{x}, \cdots, A_{\rho, 1}^{x}, D\right)
\end{aligned}
$$

where $\rho, i$ represents the $i$ th entry in a permutation $\rho$ of the integers 1 thru $K$. The greater generality of (5) in comparison to (4) can yield better fits to the data. It can be reasonably assumed that the EM algorithm under the conditional independence assumption (as the actual computational methods work) simultaneously chooses the best permutation and the best parameters. 
Because training data are seldom available and can be exceptionally expensive to obtain, some authors (Larsen and Rubin 2001) recommend semi-supervised learning where a small amount of judiciously chosen training data are combined with a large amount of unlabeled data for which true matching status is unknown. The semi-supervised methods generally outperform the unsupervised methods.

Some commercial record linkage software uses rule-based methods, which employ strategies such as if these three fields are the same in a pair of records, call the pair a designated match, designated link, or possible same entity. Ferrante and Boyd (2012), in a large comparison, showed that one rule-based commercial package was outperformed by one commercial package and several shareware packages that each applied variants of the Fellegi-Sunter model.

\section{Applications}

Record linkage has been used in a wide range of domains (Christen 2012; Herzog et al. 2007). In the following we briefly describe some example applications:

- Linking personal data: Traditionally the most common use of record linkage is to identify and link records about the same person across two databases. Examples of such linkages occur in national censuses (linking people between two census collections), in the health domain (linking patient records between different hospital and healthcare providers or over time with the aim to compile patient-oriented longitudinal data sets for public health studies), or between government agencies to, for example, identify people who commit welfare fraud.

In the health domain, population informatics (Kum et al. 2014), the study of populations by linking and analyzing large databases that contain detailed information about a large proportion of individuals in a population (such as their health, education, financial, census, location, shopping, employment, or social net- working records), has recently attracted increasing interest.

- Deduplication of customer databases: A common data quality problem for many businesses is that a customer might be recorded in their databases more than once due to address or name changes and variations. Such duplicates can incur significant costs for a business, for example, when sending out advertisement mail. The task of deduplicating a single database is in principle the same as when linking two databases. Each record in the database potentially needs to be compared with all others (indexing or blocking is generally also applied to speed up the deduplication process).

- Linking historical population data: The quantitative social sciences are currently seeing a shift toward the use of large-scale data collections for studying a diverse range of aspects of the human society. Often these are historical data such as census, birth, death, and marriage registries that span several decades (or even centuries) and that need to be linked to reconstruct historical populations (Bloothooft et al. 2015). The major challenges when linking such data include data quality (as such data have to be transcribed from hand-written forms, a process that is error-prone and labor-intensive), the dynamics of people's characteristics over time, and the complexity of roles and relationships for each individual as they change over time.

- Consumer product comparison shopping: With the increasing popularity of online comparison shopping Web sites, the challenging task of identifying which descriptions of products across diverse shopping sites correspond to the same real-world product has attracted interest from various domains. Compared to personal data, such as names and addresses, different consumer products might only be distinguishable by a single digit (such as the Canon 600D and Canon 650D digital cameras). To improve linkage quality in this domain, novel similarity calculation functions and machine learning approaches 
that learn the characteristic features that distinguish consumer products have been developed (Christen 2012).

- Linking bibliographic data: Research is increasingly being published through online databases such as Springer Link or the ACM Digital Library. These databases facilitate a much faster dissemination of knowledge, and they allow government funding agencies to calculate numerical metrics to assess the impact of researchers, research groups, and even institutions. This requires to link all records of an individual researcher with high accuracy. A major challenge with bibliographic data is that there can be several researchers with the same name details in a database, some even working in the same research domain. Even if full given names are provided, it can be unclear if two publications were written by the same individual or not. Journal and conference names are also often abbreviated and do not follow standardized formats.

\section{Future Directions}

Most research in record linkage in the past decade has concentrated on improving either the scalability of the linkage process through the development of advanced indexing or blocking techniques (Christen 2012) or linkage quality by employing sophisticated classification techniques. Most of these techniques assume the databases to be linked are static and the linkage can be done off-line and in batch mode. In the following we summarize areas of ongoing research that aim to address various practical problems in record linkage.

- Collective classification: Traditional record linkage techniques classify each compared record pair individually (Herzog et al. 2007). This can lead to violations of transitivity (if record $\mathrm{A}$ is classified as a match with record $\mathrm{B}$, and record $\mathrm{B}$ as a match with record $\mathrm{C}$, then records $\mathrm{A}$ and $\mathrm{C}$ must also be a match). With traditional approaches, transitivity is often addressed in a post-linkage process (Christen 2012).
Recently developed graph-based and collective entity resolution techniques (Bhattacharya and Getoor 2007) instead aim to find an overall optimal assignment of records to entities. These techniques take both attribute similarities and relationship information into account. They generally build a graph where nodes are records and edges connect records that have a similarity above a certain minimum threshold. The task then becomes one of splitting such a graph into individual subgraphs such that each subgraph contains the records of one entity only, and each entity is represented by one subgraph.

While such techniques have been shown to achieve high linkage quality (mainly on bibliographic data), their computational complexity (quadratic or larger in the number of records to be linked) makes the application of these techniques to large-scale record linkage problems challenging. Furthermore, how to employ such collective linkage techniques in domains where only limited relational information is available (such as for data about people) is an open question.

- Group linkage: Related to the previous topic is the challenge of linking groups of records instead of individual pairs. Groups can, for example, represent the people in a household or family or the coauthors of a scientific publication. Group linkage (On et al. 2007) is generally a two-step process, where in the first step, individual record pairs are linked, followed by the linkage of groups using some form of bipartite graph matching. The challenges in group linkage occur when groups do not have the same number of members, when group membership changes over time, and when groups can split or merge, such as does happen in families and households.

- Linking temporal data: Most personal details of people change over time, such as their addresses, names, employments, and relationships. If records with such details have time stamps attached (such as the dates when the information was recorded), then considering such temporal information might help improve linkage quality (Li et al. 2011). For ex- 
ample, if it is known that a certain proportion of people in a population move their address between two census collections 5 years apart, then less weight should be assigned to address similarities when the overall similarities between records are calculated. Some initial work has investigated how such adjustment of similarity weights can help improve overall linkage quality for bibliographic data (assuming authors change their institutions); however more research is needed to investigate if and how such techniques can be employed when, for example, linking census or health data.

- Statistical analysis across multiple files: Economists and demographers want to analyze $(\mathbf{X}, \mathbf{Y})$ - data where multivariate $\mathbf{X}$ is taken from one file $\mathbf{A}$ and multivariate $\mathbf{Y}$ is taken from another file $\mathbf{B}$. The common data for linking the files are typically nonunique identifiers such as name, address, and date of birth. Lahiri and Larsen (2005) provide such a model that can adjust certain statistical analyses such as regression under modest assumptions for linkage error. Others have considered $(\mathbf{X}, \mathbf{Y})$ - data where $\mathbf{X}$ and $\mathbf{Y}$ are each composed of discrete or continuous data but under very strong assumptions.

- Real-time linkage: As services in the public and private sectors move online, organizations increasingly require real-time linkage in applications such as online identity verification based on personal details or web and document search where duplicates in the set of retrieved records or documents need to be identified. Compared to the batch linkage of two databases, real-time linkage considers a stream of query records that need to be linked in sub-second time with a potentially large database that contains entity records. Often these databases are also dynamic, where new records are added and existing records are modified.

Novel indexing techniques are required that allow the efficient and effective retrieval of candidate records that are likely to be matching with a query record (Ramadan et al. 2015), as well as fast classification and ranking techniques that are adaptive to changes in the underlying entity database.

- Privacy-preserving record linkage: In many application domains, record linkage relies on personal details, such as names and addresses, to conduct the linkage. Privacy and confidentiality issues can be of great concern, especially when databases are linked between organizations. Many countries have privacy legislation that limits the sharing and use of personal information. Linking records, for example, between a private hospital and a government health department might therefore be limited or even prohibited.

The past decade has seen the emergence of research that aims to develop techniques that allow the linking of databases across organizations while ensuring that no sensitive private or confidential information is being revealed (Vatsalan et al. 2013). Using encoding techniques such as one-way hashing and Bloom filters, and cryptographic approaches such as secure multiparty computation, privacy-preserving record linkage techniques encode records at the data sources in such ways that similarity calculations and approximate matching of string values are feasible, while still allowing the linkage of large databases in efficient and effective ways.

\section{Cross-References}
- Classification
- Data Preparation
- Entity Resolution
- Expectation Maximization Clustering
- Link Mining and Link Discovery
- Link Prediction
- Similarity Measures
- Unsupervised Learning 


\section{Recommended Reading}

Bhattacharya I, Getoor L (2007) Collective entity resolution in relational data. ACM Trans Knowl Discov Data 1(1), 5-es, pp 1-35

Bloothooft G, Christen P, Mandemakers K, Schraagen M (2015) Population reconstruction. Springer, Cham

Christen P (2012) Data matching - concepts and techniques for record linkage, entity resolution, and duplicate detection. Data-centric systems and applications. Springer, Berlin/New York

Dempster AP, Laird NM, Rubin DB (1977) Maximum likelihood from incomplete data via the EM algorithm. J R Stat Soc Ser B 19:380-393

Dunn H (1946) Record linkage. Am J Publ Health 36(12):1412

Fellegi IP, Sunter AB (1969) A theory for record linkage. J Am Stat Assoc 64(328):1183-1210

Ferrante A, Boyd J (2012) A transparent and transportable methodology for evaluating data linkage software. J Biomed Inf 45(1):165-172

Herzog TN, Scheuren FJ, Winkler WE (2007) Data quality and record linkage techniques. Springer, New York/London

Herzog TN, Scheuren FJ, Winkler WE (2010) Record linkage. Wiley Interdiscip Rev Comput Stat 2(5): 535-543

Kum HC, Krishnamurthy A, Machanavajjhala A, Ahalt SC (2014) Social genome: putting big data to work for population informatics. IEEE Comput 47(1):5663

Lahiri P, Larsen M (2005) Regression analysis with linked data. J Am Stat Assoc 100:222-230

Larsen MD, Rubin DB (2001) Iterative automated record linkage using mixture models. J Am Stat Assoc 96(453):32-41

Li P, Dong XL, Maurino A, Srivastava D (2011) Linking temporal records. The VLDB conference was in Seattle, WA. In: Proceedings of the VLDB endowment, Seattle, vol 4, issue 11

Newcombe H, Kennedy J, Axford S, James A (1959) Automatic linkage of vital records. Science 130(3381):954-959

On BW, Koudas N, Lee D, Srivastava D (2007) Group linkage. In: IEEE international conference on data engineering, Istanbul, pp 496-505

Ramadan B, Christen P, Liang H, Gayler RW (2015) Dynamic sorted neighborhood indexing for real time entity resolution. ACM J Data Inf Qual 6(4):15

Vatsalan D, Christen P, Verykios VS (2013) A taxonomy of privacy-preserving record linkage techniques. Elsevier Inf Syst 38(6):946-969

Winkler WE (1988) Using the EM algorithm for weight computation in the Fellegi-Sunter model of record linkage. The American Statistical Associa- tion that is located in Alexandria, VA publishes the proceedings. In: Proceedings of the section on survey research methods, New Orleans, Washington, pp 667-671

\section{Recurrent Associative Memory}

Hopfield Network

\section{Recursive Partitioning}

Divide-and-Conquer Learning

\section{Reference Reconciliation}

$$
\begin{aligned}
& \text { Entity Resolution } \\
& \text { - Record Linkage }
\end{aligned}
$$

\section{Regression}

\author{
Novi Quadrianto $^{1}$ and Wray L. Buntine ${ }^{2,3}$ \\ ${ }^{1}$ Department of Informatics, SMiLe CLiNiC, \\ University of Sussex, Brighton, UK \\ ${ }^{2}$ Statistical Machine Learning Program, NICTA, \\ Canberra, ACT, Australia \\ ${ }^{3}$ Faculty of Information Technology, Monash \\ University, Clayton, VIC, Australia
}

\section{Definition}

Regression is a fundamental problem in statistics and machine learning. In regression studies, we are typically interested in inferring a realvalued function (called a regression function) whose values correspond to the mean of a dependent (or response or output) variable conditioned on one or more independent (or input) variables. Many different techniques for estimating this regression function have been developed, including parametric, semiparametric, and nonparametric methods. 


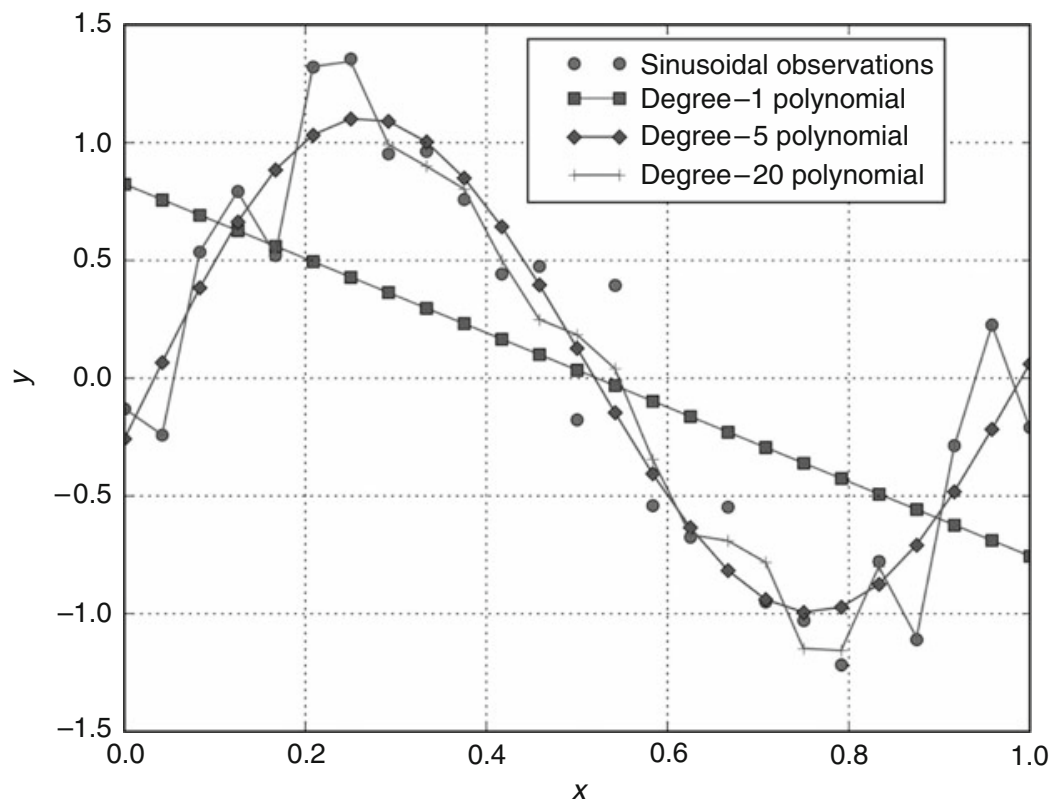

Regression, Fig. 1 Twentyfive data points (onedimensional input $x$ and output $y$ variables) with a Gaussian-corrupted sinusoidal input-output relationship, $y=\sin (2 \pi x)+\epsilon$ where $\epsilon$ is the normally distributed

\section{Motivation and Background}

Assume that we are given a set of data points sampled from an underlying but unknown distribution, each of which includes input $x$ and output $y$. An example is given in Fig. 1. The task of regression is to learn a hidden functional relationship between $x$ and $y$ from observed and possibly noisy data points. In Fig. 1, the inputoutput relationship is a Gaussian-corrupted sinusoidal relationship, that is, $y=\sin (2 \pi x)+\epsilon$ where $\epsilon$ is the normally distributed noise. Various lines show the inferred relationship based on a linear parametric regression model with polynomial basis functions. The higher the degree of the polynomial, the more complex is the inferred relationship, as shown in Fig. 1, as the function tries to better fit the observed data points.

While the most complex polynomial here is an almost perfect reconstruction of observed data points (it has "low bias"), it gives a very poor representation of the true underlying function $\sin (2 \pi x)$ that can change significantly noise. The task is to learn the functional relationship between $x$ and $y$. Various lines show the inferred relationship based on a linear regression model with polynomial basis functions having various degrees

with the change of a few data points (it has "high variance"). This phenomenon is called the bias-variance dilemma, and selecting a complex model with too high a variance is called $>$ overfitting. Complex parametric models (like polynomial regression) lead to low bias estimators with a high variance, while simple models lead to low variance estimators with high bias. To sidestep the problem of trying to estimate or select the model complexity represented, for instance, by the degree of the polynomial, so-called nonparametric methods allow a rich variety of functions from the outset (i.e., a function class not finitely parameterizable) and usually provide a hyperparameter that tunes the regularity, curvature, or complexity of the function.

\section{Theory/Solution}

Formally, in a regression problem, we are interested in recovering a functional dependency $y_{i}=f\left(x_{i}\right)+\epsilon_{i}$ from $N$-observed training data 
points $\left\{\left(x_{i}, y_{i}\right)\right\}_{i=1}^{N}$, where $y_{i} \in \mathbb{R}$ is the noisy observed output at input location $x_{i} \in \mathbb{R}^{d}$. For - linear regression, we represent the regression function $f()$ by a parameter $w \in \mathbb{R}^{H}$ in the form $f\left(x_{i}\right):=\left\langle\phi\left(x_{i}\right), w\right\rangle$ for $H$ fixed basis functions $\left\{\phi_{h}\left(x_{i}\right)\right\}_{h=1}^{H}$. With general basis functions such as polynomials, exponentials, sigmoids, or even more sophisticated Fourier or wavelets bases, we can obtain a regression function which is nonlinear with regard to the input variables although still linear with regard to the parameters.

In regression, many more methods are possible. Some variations on these standard linear models are piecewise linear models, trees, and splines (roughly, piecewise polynomial models joined up smoothly) (Hastie et al. 2003). These are called semi-parametric models, because they have a linear parametric component as well as a nonparametric component.

\section{Fitting}

In general, regression fits a model to data using an objective function or quality criterion in a form such as

$$
E(f)=\sum_{i=1}^{N} \epsilon\left(y_{i}, f\left(x_{i}\right)\right),
$$

where smaller $E(f)$ implies better quality. This might be derived as an error/loss function or as a negative log likelihood or log probability. The squared error function is the most convenient (leading to a least squares calculation), but many possibilities exist. In general, methods are distinguished by three aspects: (1) the representation of the function $f(0$, (2) the form of the term $\epsilon\left(y_{i}, f\left(x_{i}\right)\right)$, and (3) the penalty term discussed next.

\section{Regularized/Penalized Fitting}

The issue of overfitting, as mentioned already in the section Motivation and Background, is usually addressed by introducing a regularization or penalty term to the objective function. The regularized objective function is now in the form of:

$$
E_{\mathrm{reg}}=E(f)+\lambda R(f) .
$$

Here, $E(f)$ measures the quality of the solution for $f()$ on the observed data points, $R(f)$ penalizes complexity of $f()$, and $\lambda$ is called the regularization parameter which controls the relative importance between the two. Measures of function curvature, for instance, can be used for $R(f)$. In standard $\triangleright$ support vector machines, the term $E(f)$ measures the hinge loss, and penalty $R(f)$ is the sum of squares of the parameters, also used in ridge regression (Hastie et al. 2003).

\section{Bias-Variance Dilemma}

As we have seen in the previous section, the introduction of the regularization term can help avoid overfitting. However, this raises the question of determining an optimal value for the regularization parameter $\lambda$. The specific choice of $\lambda$ controls the bias-variance tradeoff (Geman et al. 1992).

Recall that we try to infer a latent regression function $f(x)$ based on $N$-observed training data points $\mathcal{D}=\left\{\left(x_{i}, y_{i}\right)\right\}_{i=1}^{N}$. The notation $f(x ; \mathcal{D})$ explicitly shows the dependence of $f$ on the data $\mathcal{D}$. The mean squared error (MSE) which measures the effectiveness of $f$ as a predictor of $y$ is

$$
\begin{aligned}
& \mathbf{E}\left[(y-f(x ; \mathcal{D}))^{2} \mid x, \mathcal{D}\right] \\
& =\mathbf{E}\left[(y-\mathbf{E}[y \mid x])^{2} \mid x, \mathcal{D}\right]+(f(x ; \mathcal{D})-\mathbf{E}[y \mid x])^{2}
\end{aligned}
$$

where $\mathbf{E}[$.] means expectation with respect to a conditional distribution $p(y \mid x)$. The first term of (2) does not depend on $f(x ; \mathcal{D})$, and it represents the intrinsic noise on the data. The MSE of $f$ as an estimator of the regression $\mathbf{E}[y \mid x]$ is

$$
\mathbf{E}_{\mathcal{D}}\left[(f(x ; \mathcal{D})-\mathbf{E}[y \mid x])^{2}\right]
$$

where $\mathbf{E}_{\mathcal{D}}$ means expectation with respect to the training set $\mathcal{D}$. The estimation error in (3) can be decomposed into a bias and a variance terms, that is, 


$$
\begin{aligned}
\mathbf{E}_{\mathcal{D}} & {\left[(f(x ; \mathcal{D})-\mathbf{E}[y \mid x])^{2}\right]=\mathbf{E}_{\mathcal{D}}[(f(x ; \mathcal{D})} \\
& \left.\left.-\mathbf{E}_{\mathcal{D}}[f(x ; \mathcal{D})]+\mathbf{E}_{\mathcal{D}}[f(x ; \mathcal{D})]-\mathbf{E}[y \mid x]\right)^{2}\right] \\
= & \mathbf{E}_{\mathcal{D}}\left[\left(f(x ; \mathcal{D})-\mathbf{E}_{\mathcal{D}}[f(x ; \mathcal{D})]\right)^{2}\right] \\
& +\left(\mathbf{E}_{\mathcal{D}}[f(x ; \mathcal{D})]-\mathbf{E}[y \mid x]\right)^{2}+2 \mathbf{E}_{\mathcal{D}}[(f(x ; \mathcal{D}) \\
& \left.\left.-\mathbf{E}_{\mathcal{D}}[f(x ; \mathcal{D})]\right)\right]\left(\mathbf{E}_{\mathcal{D}}[f(x ; \mathcal{D})]-\mathbf{E}[y \mid x]\right) \\
= & \mathbf{E}_{\mathcal{D}}\left[\left(f(x ; \mathcal{D})-\mathbf{E}_{\mathcal{D}}[f(x ; \mathcal{D})]\right)^{2}\right] \\
& +\left(\mathbf{E}_{\mathcal{D}}[f(x ; \mathcal{D})]-\mathbf{E}[y \mid x]\right)^{2} \\
= & \operatorname{variance}+\text { bias }^{2} .
\end{aligned}
$$

The bias term measures the difference between the average predictor over all datasets and the desired regression function. The variance term measures the adaptability of the predictor to a particular dataset. There is a tradeoff between the bias and variance contributions to the estimation error, with very flexible models having low bias but high variance (overfitting) and relatively rigid models having low variance but high bias (underfitting). Typically, variance is reduced through "smoothing," that is, an introduction of the regularization term. This, however, will introduce bias as peaks and valleys of the regression function will be blurred. To achieve an optimal predictive capability, an estimator with the best balance between bias and variance is chosen by varying the regularization parameter $\lambda$. It is crucial to note that bias-variance decomposition albeit powerful is based on averages of datasets; however, in practice only a single dataset is observed. In this regard, a Bayesian treatment of regression, such as Gaussian process regression which will avoid overfitting problem of maximum likelihood and which will also lead to automatic methods of determining model complexity using the training data alone, could be an attractive alternative.

\section{Nonparametric Regression}

In the parametric approach, an assumption on the mathematical form of the functional relationship between input $x$ and output $y$ such as linear, polynomial, exponential, or combination of them needs to be chosen a priori. Subsequently, parameters are placed on each of the chosen forms and the optimal values learned from the observed data. This is restrictive both in the fixed functional form and in the ability to vary the model complexity. Nonparametric approaches try to derive the functional relationship directly from the data, that is, they do not parameterize the regression function.

- Gaussian Processes for regression, for instance, are well developed. Another approach is the kernel method, of which a rich variety exists (Hastie et al. 2003). These can be viewed as a regression variant of nearest neighbor classification where the function is made up of a local element for each data point:

$$
f(x)=\frac{\sum_{i} y_{i} K_{\lambda}\left(x_{i}, x\right)}{\sum_{i} K_{\lambda}\left(x_{i}, x\right)},
$$

where the function $K_{\lambda}\left(x_{i},\right)$ is a nonnegative "bump" in $x$ space centered at its first argument with diameter approximately given by $\lambda$. Thus, the function has a variable contribution from each data point and $\lambda$ controls the bias-variance tradeoff.

\section{Generalized Linear Models}

The previous discussion about regression focuses on continuous output/dependent variables. While this type of regression problem is ubiquitous, there are however some interests in cases of restricted output variables:

1. The output variable consists of two categories (called binomial regression).

2. The output variable consists of more than two categories (called multinomial regression).

3. The output variable consists of more than two categories which can be ordered in a meaningful way (called ordinal regression). and

4. The output variable is a count of the repetition of the occurrence of an event (called poisson regression).

Nelder and Wedderburn (1972) introduced the generalized linear model (GLM) by allowing the linear model to be related to the output variables via a link function. This is a way to unify different cases of response variables under one 
Regression, Table 1 A table of various link functions associated with the assumed distribution on the output variable

\begin{tabular}{l|l|l}
\hline $\begin{array}{l}\text { Distribution of } \\
\text { dependent variable }\end{array}$ & Name & Link function \\
\hline Gaussian & Identity link & $g(\mu)=\mu$ \\
\hline Poisson & Log link & $g(\mu)=\log (\mu)$ \\
\hline $\begin{array}{l}\text { Binomial } \\
\text { multinomial }\end{array}$ & Logit link & $g(\mu)=\log \left(\frac{\mu}{1-\mu}\right)$ \\
\hline $\begin{array}{l}\text { Exponential } \\
\text { gamma }\end{array}$ & Inverse link & $g(\mu)=\mu^{-1}$ \\
\hline $\begin{array}{l}\text { Inverse Gaussian } \\
\text { Inverse } \\
\text { squared link }\end{array}$ & $g(\mu)=\mu^{-2}$ \\
\hline
\end{tabular}

framework, each only differs in the choice of the link function. Specifically, in GLM, each output variable is assumed to be generated from the exponential family of distributions. The mean of this distribution depends on the input variables through

$\mathbf{E}[y]=g(\mu)=w_{0}+w_{1} \phi_{1}\left(x_{i}\right)+\ldots+w_{D} \phi_{D}\left(x_{i}\right)$,

where $g(\mu)$ is the link function (Table 1). The parameters of the generalized linear model can then be estimated by the maximum likelihood method, which can be found by iterative reweighted least squares (IRLS), an instance of the expectation maximization (EM) algorithm.

\section{Other Variants of Regression}

So far, we have focused on the problem of predicting a single output variable $y$ from an input variable $x$. Some studies look at predicting multiple output variables simultaneously. The simplest approach for the multiple outputs problem would be to model each output variable with a different set of basis functions. The more common approach uses the same set of basis functions to model all of the output variables. Not surprisingly, the solution to the multiple outputs problem decouples into independent regression problems with shared basis functions.

For some other studies, the focus of regression is on computing several regression functions corresponding to various percentage points or quantiles (instead of the mean) of the conditional distribution of the dependent variable given the independent variables. This type of regression is called quantile regression (Koenker 2005). The sum of tilted absolute loss (called pinball loss) is being optimized for this type of regression. Quantile regression has many important applications within econometrics, data mining, social sciences, and ecology, among other domains.

Instead of inferring one regression function corresponding to the mean of a response variable, $k$ regression functions can be computed with the assumption that the response variable is generated by a mixture of $k$ components. This is called the mixture of regressions problem (Gaffney and Smyth 1999). Applications include trajectory clustering, robot planning, and motion segmentation.

Another important variant is the heteroscedastic regression model where the noise variance on the data is a function of the input variable $x$. The Gaussian process framework can be used conveniently to model this noise-dependent case by introducing a second Gaussian process to model the dependency of noise variance on the input variable (Goldberg et al. 1998). There are also attempts to make the regression model more robust to the presence of a few problematic data points called outliers. The sum of absolute loss (instead of the sum of squared loss) or student's t-distribution (instead of Gaussian distribution) can be used for robust regression.

\section{Cross-References}

\author{
- Gaussian Processes \\ - Linear Regression \\ - Support Vector Machines
}

\section{Recommended Reading}

Machine learning textbooks such as Bishop (2006), among others, introduce different regression models. For a more statistical introduction including an extensive overview of the many different semi-parametric methods and non-parametric methods such as kernel methods, see Hastie et al. (2003). For a coverage 
of key statistical issues including nonlinear regression, identifiability, measures of curvature, autocorrelation, and such, see Seber and Wild (1989). For a large variety of built-in regression techniques, refer to R (http://www.r-project.org/).

Bishop C (2006) Pattern recognition and machine learning. Springer, New York

Gaffney S, Smyth P (1999) Trajectory clustering with mixtures of regression models. In: ACM SIGKDD, vol 62. ACM, New York, pp 63-72

Geman S, Bienenstock E, Doursat R (1992) Neural networks and the bias/variance dilemma. Neural Comput 4:1-58

Goldberg P, Williams C, Bishop C (1998) Regression with input-dependent noise: a Gaussian process treatment. In: Neural information processing systems, vol 10. MIT

Hastie T, Tibshirani R, Friedman J (Corrected ed) (2003) The elements of statistical learning: data mining, inference, and prediction. Springer, New York

Koenker R (2005) Quantile regression. Cambridge University Press, Cambridge

Nelder JA, Wedderburn RWM (1972) Generalized linear models. J R Stat Soc: Ser A 135: 370-384

Seber G, Wild C (1989) Nonlinear regression. Wiley, New York

\section{Regression Trees}

\section{Luís Torgo}

University of Porto, Porto, Portugal

\section{Synonyms}

Decision trees for regression; Piecewise constant models; Tree-based regression

\section{Definition}

Regression trees are supervised learning methods that address multiple regression problems. They provide a tree-based approximation $\hat{f}$, of an unknown regression function $Y=f(\mathbf{x})+\varepsilon$ with $Y \in \Re$ and $\varepsilon \approx N\left(0, \sigma^{2}\right)$, based on a given sample of data $D=\left\{\left\langle x_{i}^{1}, \cdots, x_{i}^{p}, y_{i}\right\rangle\right\}_{i=1}^{n}$. The obtained models consist of a hierarchy of logical tests on the values of any of the $p$ predictor vari- ables. The terminal nodes of these trees, known as the leaves, contain the numerical predictions of the model for the target variable $Y$.

\section{Motivation and Background}

Work on regression trees goes back to the AID system by Morgan and Sonquist (1963). Nonetheless, the seminal work is the book Classification and Regression Trees by Breiman and colleagues (1984). This book has established several standards in many theoretical aspects of tree-based regression, including over-fitting avoidance by post-pruning, the notion of surrogate splits for handling unknown variable, and estimating variable importance.

Regression trees have several features that make them a very interesting approach to several multiple regression problems. Namely, regression trees provide (i) automatic variable selection making them highly insensitive to irrelevant variables, (ii) computational efficiency that allows addressing large problems, (iii) handling of unknown variable values, (iv) handling of both numerical and nominal predictor variables, (v) insensitivity to predictors' scales, and (vi) interpretable models for most domains. In spite of all these advantages, regression trees have poor prediction accuracy in several domains because of the piecewise constant approximation they provide, and they are also unstable with respect to small changes on the training data.

\section{Structure of Learning System}

The most common regression trees are binary with logical tests in each node (an example is given on the left graph of Fig. 1). Tests on numerical variables usually take the form $x_{i}<\alpha$, with $\alpha \in \Re$, while tests on nominal variables have the form $x_{j} \in\left\{v_{1}, \cdots, v_{m}\right\}$. Each path from the root (top) node to a leaf can be seen as a logical assertion defining a region on the predictors' space. Any regression tree provides a full mutually exclusive partition of the predictor space into $L$ regions with boundaries that are 

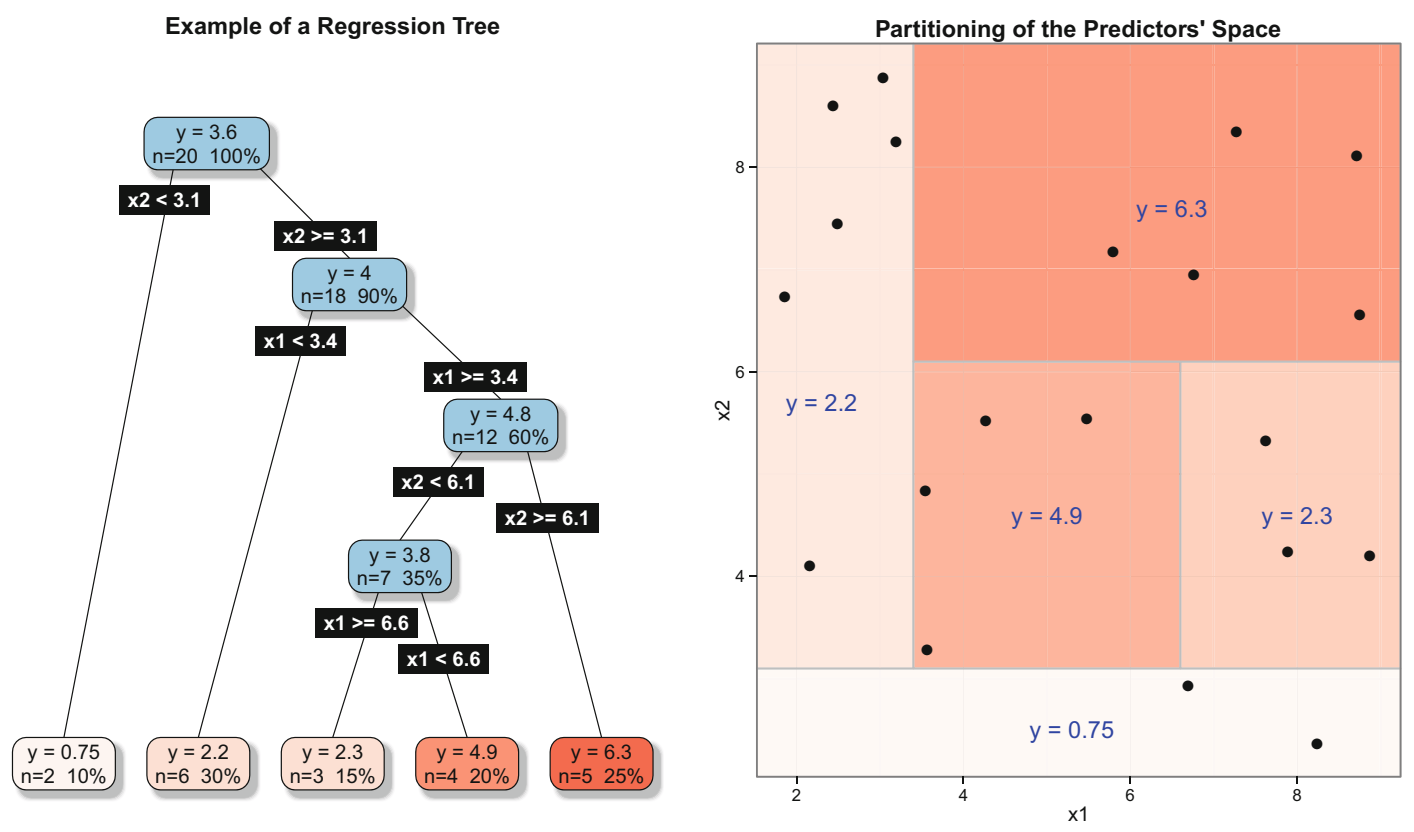

Regression Trees, Fig. 1 A regression tree and the partitioning it provides

parallel to the predictors' axes due to the form of the tests. Figure 1 illustrates these ideas with a tree and the respective partitioning on the right side of the graph.

Using a regression tree for obtaining predictions for new observations is straightforward. For each new observation, we follow a path from the root node to a leaf selecting the branches according to the variable values of the observation. All observations falling in a partition are predicted with the same constant value, and that is the reason for regression trees sometimes being referred to as piecewise constant models. In effect, the approximation provided by a regression tree is given by

$$
Y=\sum_{l \in \mathcal{L}} k_{l} \cdot I\left(P_{l}\right)
$$

where $\mathcal{L}$ is the set of $L$ leaves, the $k \mathrm{~s}$ are the constants at each leaf, $I()$ is an indicator function, and $P_{i}$ is a logical assertion formed by the conjunction of conditions from the root node till the leaf $i$. For instance, the rightmost leaf of the tree in Fig. 1 is described by the logical assertion $x_{2} \geq 3.1 \wedge x_{1} \geq 3.4 \wedge x_{2} \geq 6.1$, which is equivalent to $x_{1} \geq 3.4 \wedge x_{2} \geq 6.1$.

\section{Learning a Regression Tree}

A binary regression tree is obtained by a very efficient algorithm known as recursive partitioning (Algorithm 1).

If the termination criterion is not met by the input sample $D$, the algorithm selects the best logical test on one of the predictor variables according to some criterion. This test divides the current sample in two partitions: the one with the cases satisfying the test and the remaining. The algorithm proceeds by recursively applying the same method to these two partitions to obtain the left and right branches of the node. Algorithm 1 has three main components that characterize the type of regression tree we are obtaining: (i) the termination criterion, (ii) the constant $k$, and (iii) the method to find the best test on one of the predictors. The choices for these components are related to the preference criteria that are used to build the trees. The most common criterion is the minimization of the sum of the square errors, known as the least squares (LS) criterion. Using this criterion it can be easily proven (e.g., Breiman et al. 1984) that the constant $k$ should be the average target variable value of the cases in the leaf. With respect to 


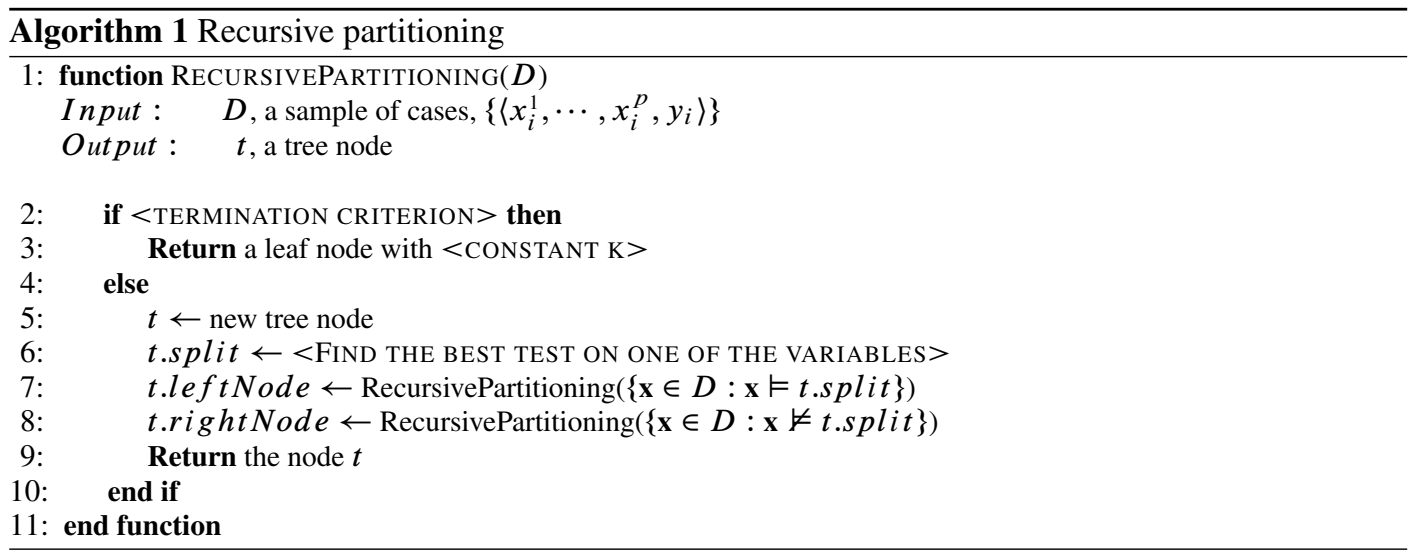

the termination criterion, usually very relaxed settings are selected so that an overly large tree is grown. The reasoning is that the trees will be pruned afterward with the goal of overcoming the problem of over-fitting of the training data.

According to the LS criterion, the error in a given node is given by

$$
\operatorname{Err}(t)=\frac{1}{n_{t}} \sum_{\left\langle\mathbf{x}_{i}, y_{i}\right\rangle \in D_{t}}\left(y_{i}-k_{t}\right)^{2}
$$

where $D_{t}$ is the sample of cases in node $t, n_{t}$ is the cardinality of this set, and $k_{t}$ is the average target variable value of the cases in $D_{t}$.

Any logical test $s$ divides the cases in $D_{t}$ in two partitions, $D_{t_{L}}$ and $D_{t_{R}}$. The resulting pooled error is given by

$$
\operatorname{Err}(t, s)=\frac{n_{t_{L}}}{n_{t}} \times \operatorname{Err}\left(t_{L}\right)+\frac{n_{t_{R}}}{n_{t}} \times \operatorname{Err}\left(t_{R}\right)
$$

where $n_{t_{L}} / n_{t}\left(n_{t_{R}} / n_{t}\right)$ is the proportion of cases going to the left (right) branch of $t$.

In this context, we can estimate the value of the split $s$ by the respective error reduction, and this can be used to evaluate all candidate splits test for a node:

$$
\Delta(s, t)=\operatorname{Err}(t)-\operatorname{Err}(t, s)
$$

Finding the best split test for a node $t$ involves evaluating all possible tests for this node using Eq.4. For each predictor of the problem, one needs to evaluate all possible splits in that variable. For continuous variables, this requires a sorting operation on the values of this variable occurring in the node. After this sorting, a fast incremental algorithm can be used to find the best cut point value for the test (e.g., Torgo 1999). With respect to nominal variables, Breiman and colleagues (1984) have proved a theorem that avoids trying all possible combinations of values, reducing the computational complexity of this task from $O\left(2^{v-1}-1\right)$ to $O(v-1)$, where $v$ is the number of values of the nominal variable.

Departures from the standard learning procedure described above include, among others, the use of multivariate split nodes (e.g., Breiman et al. 1984, Li et al. 2000, and Gama 2004) to overcome the axis parallel representation limitation of partitions, the use of different criteria to find the best split node (e.g., Robnik-Sikonja and Kononenko 1996, Buja and Lee 2001, and Loh 2002), the use of different preference criteria to guide the tree growth (e.g., Breiman et al. 1984, Torgo 1999, Buja and Lee 2001, and Torgo and Ribeiro 2003), and the use of both regression and split nodes (e.g., Lubinsky 1995 and Malerba et al. 2004).

\section{Pruning Regression Trees}

As most nonparametric modeling techniques, regression trees may over-fit the training data which will inevitably lead to poor out of the sample 
predictive performance. The standard procedure to fight this undesirable effect is to grow an overly large tree and then to use some reliable error estimation procedure to find the "best" sub-tree of this large model. This procedure is known as post-pruning a tree (Breiman et al. 1984). An alternative is to stop tree growth sooner in a process known as pre-pruning which again needs to be guided by reliable error estimation to known when over-fitting is starting to occur. Although more efficient in computational terms, this latter alternative may lead to stop tree growth too soon even with look-ahead mechanisms.

Post-pruning is usually carried out in a threestage procedure: (i) a set of sub-trees of the initial tree is generated, (ii) some reliable error estimation procedure is used to obtain estimates for each member of this set, and (iii) some method based on these estimates is used to select one of these trees as the final tree model. Different methods exist for each of these steps. A common setup (e.g., Breiman et al. 1984) is to use errorcomplexity pruning to generate a sequence of nested sub-trees, whose error is then estimated by cross validation. The final tree is selected using the $x$-SE rule which starts with the lowest estimated error sub-tree and then selects the smallest tree within the interval of $x$ standard errors of the lowest estimated error tree (a frequent setting is to use 1 standard error).

Variations on the subject of pruning regression trees include, among others, pre-pruning alternatives (e.g., Breiman and Meisel 1976 and Friedman 1979), the use of different tree error estimators (see Torgo (1998) for a comparative study and references to different alternatives), and the use of the MDL principle to guide the pruning (Robnik-Sikonja and Kononenko 1998).

\section{Cross-References}

\author{
- Model Trees \\ $>$ Random Forests \\ - Regression \\ $\checkmark$ Supervised Learning
}

\section{Recommended Reading}

Breiman L, Friedman J, Olshen R, Stone C (1984) Classification and regression trees. Statistics/probability series. Wadsworth \& Brooks/Cole Advanced Books \& Software, Belmont

Breiman L, Meisel WS (1976) General estimates of the intrinsic variability of data in nonlinear regression models. J Am Stat Assoc 71:301-307

Buja A, Lee Y-S (2001) Data mining criteria for treebased regression and classification. In: Proceedings of ACM SIGKDD international conference on knowledge discovery and data mining, San Francisco, pp 27-36

Friedman JH (1979) A tree-structured approach to nonparametric multiple regression. In: Gasser $\mathrm{T}$, Rosenblatt M (eds) Smoothing techniques for curve estimation. Lecture notes in mathematics, vol 757. Springer, Berlin/New York, pp 5-22

Gama J (2004) Functional trees. Mach Learn 55(3):219-250

Li KC, Lue H, Chen C (2000) Interactive treestructured regression via principal Hessians direction. J Am Stat Assoc 95:547-560

Loh W (2002) Regression trees with unbiased variable selection and interaction detection. Stat Sin 12: 361-386

Lubinsky D (1995) Tree structured interpretable regression. In: Proceedings of the workshop on AI \& statistics, Key West

Malerba D, Esposito F, Ceci M, Appice A (2004) Top-down induction of model trees with regression and splitting nodes. IEEE Trans Pattern Anal Mach Intell 26(5):612-625

Morgan JN, Sonquist JA (1963) Problems in the analysis of survey data, and a proposal. J Am Stat Assoc 58(302):415-434

Robnik-Sikonja M, Kononenko I (1996) Contextsensitive attribute estimation in regression. In: Proceedings of the ICML-96 workshop on learning in context-sensitive domains, Bari

Robnik-Sikonja M, Kononenko I (1998) Pruning regression trees with MDL. In: Proceedings of ECAI98, Brighton

Torgo L (1998) Error estimates for pruning regression trees. In: Nedellec C, Rouveirol C (eds) Proceedings of the 10th European conference on machine learning, Chemnitz. LNAI, vol 1398. Springer

Torgo L (1999) Inductive learning of tree-based regression models. PhD thesis, Faculty of Sciences, Department of Computer Science, University of Porto

Torgo L, Ribeiro R (2003) Predicting outliers. In: Lavrac N, Gamberger D, Todorovski L, Blockeel $\mathrm{H}$ (eds) Proceedings of principles of data mining and knowledge discovery (PKDD'03), Cavtat/Dubronik. LNAI, vol 2838. Springer, pp 447-458 


\section{Regularization}

Xinhua Zhang

NICTA, Australian National University, Canberra, ACT, Australia

School of Computer Science, Australian

National University, Canberra, ACT, Australia

NICTA London Circuit, Canberra, ACT,

Australia

\section{Abstract}

Regularization plays a key role in many machine learning algorithms. Exactly fitting a model to the training data is generally undesirable, because it will fit the noise in the training examples ( $\triangleright$ overfitting), and is doomed to predict (generalize) poorly on unseen data. In contrast, a simple model that fits the training data well is more likely to capture the regularities in it and generalize well. A number of regularizers have been proposed for various applications, and theoretical tools that characterize their complexity are also available.

\section{Definition}

In general, a regularizer a quantifier of the complexity of a model, and many successful machine learning algorithms fall in the framework of regularized risk minimization:

(How well the model fits the training data)

$+\lambda \cdot($ complexity/regularization of the model $)$,

where the positive real number $\lambda$ controls the trade-off.

There is a variety of regularizers, which yield different statistical and computational properties. In general, there is no universally best regularizer, and a regularization approach must be chosen depending on the dataset.

\section{Motivation and Background}

The main goal of machine learning is to induce a model from the observed data and use this model to make predictions and decisions. This is also largely the goal of general natural science and is commonly called inverse problems ("forward problem" means using the model to generate observations). Therefore, it is no surprise that regularization had been well studied before the emergence of machine learning.

Inverse problems are typically ill posed, e.g., having only a finite number of samples drawn from an uncountable space or having a finite number of measurements in an infinite dimensional space. In machine learning, we often need to induce a classifier for the whole feature-label space, while only a finite number of feature-label pairs are available for training. In practice, the set of candidate models is often flexible enough to precisely fit all the training examples. However, this can lead to significant overfitting when the training data is noisy, and the real challenge is how to generalize well on the unseen data in the whole feature-label space.

Many techniques have been proposed to tackle ill-posed inverse problems. Almost all of them introduce an additional measure on how much a model is preferred a priori (i.e., without observing the training data). This extra belief on the desirable form of the model reflects the external knowledge of the model designer. It cannot be replaced by the data itself according to the "no free lunch theorem," which states that if there is no assumption on the mechanism of labeling, then it is impossible to generalize, and any model can be inferior to another on some distribution of the feature-label pair (Devroye et al. 1996).

A commonly used prior is the so-called > Occam's razor, which prefers "simple" models. It asserts that among all the models which fit the training data well, the simplest one is more likely to capture the "regularities" in it and hence has a larger chance to generalize well to the unseen data. Then an immediate question is how to quantify the complexity of a model, which is often called a regularizer. Intuitively, a regularizer can encode preference for a sparse 
model (few features are relevant for prediction), a large margin model (two classes have a wide margin), or a smooth model with weak highfrequency components. A general framework of regularization was given by Tikhonov (1943).

\section{Theory}

Suppose $n$ feature-label pairs $\left\{\left(\mathbf{x}_{i}, y_{i}\right)\right\}_{i=1}^{n}$ are drawn iid from a certain joint distribution $P$ on $\mathcal{X} \times \mathcal{Y}$, where $\mathcal{X}$ and $\mathcal{Y}$ are the spaces of feature and label, respectively. Let the marginal distribution on $\mathcal{X}$ and $\mathcal{Y}$ be $P_{x}$ and $P_{y}$ respectively. For convenience, let $\mathcal{X}$ be $\mathbb{R}^{p}$ (Euclidean space). Denote $X:=\left(\mathbf{x}_{1}, \ldots, \mathbf{x}_{n}\right)$ and $\mathbf{y}:=$ $\left(y_{1}, \ldots, y_{n}\right)^{\top}$.

\section{An Illustrative Example: Ridge Regression}

Ridge regression is illustrative of the use of regularization. It tries to fit the label $y$ by a linear model $\langle\mathbf{w}, \mathbf{x}\rangle$ (inner product). So we need to solve a system of linear equations in $\mathbf{w}:\left(\mathbf{x}_{1}, \ldots, \mathbf{x}_{n}\right)^{\top} \mathbf{w}=\mathbf{y}$, which is equivalent to a linear least square problem: $\min _{\mathbf{w} \in \mathbb{R}^{p}}\left\|X^{\top} \mathbf{w}-\mathbf{y}\right\|^{2}$. If the rank of $X$ is less than the dimension of $\mathbf{w}$, then it is overdetermined and the solution is not unique.

To approach this ill-posed problem, one needs to introduce additional assumptions on what models are preferred, i.e., the regularizer. One choice is to pick a matrix $\Gamma$ and regularize $\mathbf{w}$ by $\|\Gamma \mathbf{w}\|^{2}$. As a result we solve $\min _{\mathbf{w} \in \mathbb{R}^{p}}\left\|X^{\top} \mathbf{w}-\mathbf{y}\right\|^{2}+\lambda\left\|\Gamma^{\top} \mathbf{w}\right\|^{2}$, and the solution has a closed form $\mathbf{w}^{*}=\left(X X^{\top}+\right.$ $\left.\lambda \Gamma \Gamma^{\top}\right) X \mathbf{y}$. $\Gamma$ can be simply the identity matrix which encodes our preference for small norm models.

The use of regularization can also be justified from a Bayesian point of view. Treating $\mathbf{w}$ as a multivariate random variable and the likelihood as $\exp \left(-\left\|X^{\top} \mathbf{w}-\mathbf{y}\right\|^{2}\right)$, then the minimizer of $\left\|X^{\top} \mathbf{w}-\mathbf{y}\right\|^{2}$ is just a maximum likelihood estimate of $\mathbf{w}$. However, we may also assume a prior distribution over w, e.g., a Gaussian prior $p(\mathbf{w}) \sim \exp \left(-\lambda\left\|\Gamma^{\top} \mathbf{w}\right\|^{2}\right)$. Then the solution of the ridge regression is simply the maximum a posterior estimate of $\mathbf{w}$.

\section{Examples of Regularization}

A common approach to regularization is to penalize a model by its complexity measured by some real-valued function, e.g., a certain "norm" of w. We list some examples below.

$\boldsymbol{L}_{1}$ regularization $L_{1}$ regularizer, $\|\mathbf{w}\|_{1}:=$ $\sum_{i}\left|w_{i}\right|$, is a popular approach to finding sparse models, i.e., only a few components of $\mathbf{w}$ are nonzero, and only a corresponding small number of features are relevant to the prediction. A well-known example is the LASSO algorithm (Tibshirani 1996), which uses a $L_{1}$-regularized least square:

$$
\min _{\mathbf{w} \in \mathbb{R}^{p}}\left\|X^{\top} \mathbf{w}-\mathbf{y}\right\|^{2}+\lambda\|\mathbf{w}\|_{1} .
$$

$\boldsymbol{L}_{\mathbf{2}}$ regularization The $L_{2}$ regularizer, $\|\mathbf{w}\|_{2}:=$ $\sqrt{\sum_{i}\left|w_{i}\right|^{2}}$, is popular due to its self-dual properties. In all $L_{p}$ spaces, only the $L_{2}$ space is Hilbertian and self-adjoint, so it affords much convenience in studying and exploiting the dual properties of the $L_{2}$-regularized models. A well-known example is the support vector machines (SVMs), which minimize the $L_{2}$-regularized hinge loss:

$$
\frac{1}{n} \sum_{i=1}^{n} \max \left\{0,1-\left\langle\mathbf{w}, \mathbf{x}_{i}\right\rangle\right\}+\lambda\|\mathbf{w}\|_{2}^{2} .
$$

$L_{p}$ regularization In general, all $L_{p}$ norms $\|\mathbf{w}\|_{p}:=\left(\sum_{i}\left|w_{i}\right|^{p}\right)^{1 / p}(p \geq 1)$ can be used for regularization. When $p<1,\left(\sum_{i}\left|w_{i}\right|^{p}\right)^{1 / p}$ is no longer convex. A specially interesting case is when $p=0$, and $\|\mathbf{w}\|_{0}$ is defined as the number of nonzero elements in $\mathbf{w}$ (the sparseness of $\mathbf{w}$ ). But explicitly optimizing the $L_{0}$ norm leads to a combinatorial problem which is hard to solve. In some cases, the $L_{1}$ regularizer can approximately recover the solution of $L_{0}$ regularization (Candes and Tao 2005).

$\boldsymbol{L}_{\boldsymbol{p}, \boldsymbol{q}}$ regularizer The $L_{p, q}$ regularizer is popular in the context of multitask learning (Tropp 2006). Suppose there are $T$ tasks, and each training example $\mathbf{x}_{i}$ has a label vector $\mathbf{y}_{i} \in \mathbb{R}^{T}$ with each component corresponding to a task. 
For each task $t$, we seek for a linear regressor $\left\langle\mathbf{w}_{t}, \mathbf{x}\right\rangle$ such that for each training example $\mathbf{x}_{i}$, $\left\langle\mathbf{w}_{t}, \mathbf{x}_{i}\right\rangle$ fits the $t$-th component of $\mathbf{y}_{i}$. Of course, the $\mathbf{w}_{t}$ could be determined independently from each other. But in many applications, the $T$ tasks are somehow related, and it will be advantageous to learn them as a whole. Stack $\mathbf{w}_{t}$ 's into a matrix $W:=\left(\mathbf{w}_{1}, \ldots, \mathbf{w}_{T}\right)$ where each column corresponds to a task and each row corresponds to a feature. Then the intuition of multitask learning can be concretized by regularizing $W$ with the $L_{p, q}$ compositional norm $(p, q \geq 1)$ :

$$
\|W\|_{p, q}:=\left(\sum_{i}\left(\sum_{t}\left|w_{i t}\right|^{p}\right)^{\frac{q}{p}}\right)^{\frac{1}{q}},
$$

where $w_{i t}$ is the $i$-th component of $\mathbf{w}_{t}$. When $q=1$, it becomes the $L_{1}$ norm of the $L_{p}$ norm of the rows, and the sparse inducing property of $L_{1}$ norm encourages the rows to have $L_{p}$ norm 0 , i.e., the corresponding feature is not used by any task. Other choices of $p$ and $q$ are also possible.

Entropy regularizer The entropy regularizer is useful in boosting, and it works in a slightly different way from the above regularizers. Boosting aims to find a convex combination of hypotheses, such that the training data is accurately classified by the ensemble. At each step, the boosting algorithm maintains a distribution $\mathbf{d}\left(d_{i}>0\right.$ and $\left.\sum_{i} d_{i}=1\right)$ over the training examples, feeds $\mathbf{d}$ to an oracle which returns a new hypothesis, and then updates $\mathbf{d}$ and go on. As a "simple" ensemble means a small number of weak hypotheses, the boosting algorithm is expected to find an accurate ensemble by taking as few steps as possible. This can be achieved by exponentiated gradient descent (Kivinen and Warmuth 1997), which stems from the relative entropy regularizer $\sum_{i} d_{i} \log \frac{d_{i}}{1 / n}$ applied at each step. It also attracts d toward the uniform distribution, which helps avoid overfitting the noise, i.e., trying hard to match the (incorrect) label of a few training examples.
Miscellaneous Instead of using a function that directly measures the complexity of the model $\mathbf{w}$, regularization can also be achieved by penalizing the complexity of the output of the model on the training data. This is called value regularization (Rifkin and Lippert 2007). It not only yields neat derivations of standard algorithms but also provides much convenience in studying the learning theory and optimization.

Furthermore, the regularized risk minimization framework in (1) is not the only approach to regularization. For example, in online learning where the model is updated iteratively, early stopping is an effective form of regularization, and it has been widely used in training neural networks. Suppose the available dataset is divided into a training set and a validation set and the model is learned online from the training set, then the algorithm terminates when the performance of the model on the validation set stops improving.

\section{Measuring the Capacity of Model Class}

Besides penalizing the complexity of the model, we can restrict the complexity of the model class $\mathcal{F}$ in the first place. For example linear regression is intrinsically "simpler" than quadratic regression. Decision stumps are "simpler" than linear classifiers. In other words, regularization can be achieved by restricting the capacity of the model class, and the key question is how to quantify this capacity. Some commonly used measures in the context of binary classification are the following:

\section{VC dimension The Vapnik-Chervonenkis} dimension ( $\vee \mathrm{VC}$ dimension) quantifies how many data points can be arbitrarily labeled by using the functions in $\mathcal{F}$ (Vapnik and Chervonenkis 1971). $\mathcal{F}$ is said to shatter a set of data points $\mathbf{x}_{1}, \ldots, \mathbf{x}_{n}$ if, for any assignment of labels to these points, there exists a function $f \in \mathcal{F}$ which yields this labeling. The VC dimension of $\mathcal{F}$ is the maximum $n$ such that any $n$ data points can be shattered by $\mathcal{F}$. For example, decision stumps have VC dimension 2, and linear classifiers (with bias) in a $p$ dimensional space have VC dimension $p+1$. 
Covering number The idea of covering number (Guo et al. 1999) is to characterize the inherent "dimension" of $\mathcal{F}$, in a way that follows the standard concept of vector dimension. Given $n$ data points $\mathbf{x}_{1}, \ldots, \mathbf{x}_{n}$, we may endow the model class $\mathcal{F}$ with the following metric:

$d_{n}(f, g):=\frac{1}{n} \sum_{i=1}^{n} \delta\left(f\left(\mathbf{x}_{i}\right) \neq g\left(\mathbf{x}_{i}\right)\right), \forall f, g \in \mathcal{F}$,

where $\delta(\cdot)=1$ if $\cdot$ is true and 0 otherwise. A set of functions $f_{1}, \ldots, f_{m}$ is said to be a cover of $\mathcal{F}$ at radius $\epsilon$ if, for any function $f \in \mathcal{F}$, there exists an $f_{i}$ such that $d_{n}\left(f, f_{i}\right)<\epsilon$. Then the covering number of $\mathcal{F}$ at radius $\epsilon>0$ with respect to $d_{n}$ is the minimum size of a cover of radius $\epsilon$.

To understand the motivation of the definition, consider the unit ball in $\mathbb{R}^{p}$. To cover it by $\epsilon$ radius balls, one needs order $N(\epsilon, p)=\epsilon^{-p}$ balls. Then the dimension $p$ can be estimated from the rate of growth of $\log N(\epsilon, p)=-p \log \epsilon$ with respect to $\epsilon$. The covering number is an analogy of $N(\epsilon, p)$, and the dimension of $\mathcal{F}$ can be estimated in the same spirit.

Rademacher average The Rademacher average is a soft variant of the VC dimension. Instead of requiring the model class to shatter $n$ data points, it allows that the labels be violated at some cost. Let $\sigma_{i} \in\{-1,1\}$ be an arbitrary assignment of the labels, and assume all functions in $\mathcal{F}$ range in $\{-1,1\}$ (this restriction can be relaxed). Then a model $f \in \mathcal{F}$ is considered as the most consistent with $\left\{\sigma_{i}\right\}$ if it maximizes $\frac{1}{n} \sum_{i=1}^{n} \sigma_{i} f\left(\mathbf{x}_{i}\right)$. This term equals 1 if $\mathcal{F}$ does contain a model consistent with $\left\{\sigma_{i}\right\}$. Then we take an average over all possible assignments of $\sigma_{i}$, i.e., treating $\sigma_{i}$ as a binary random variable with $P\left(\sigma_{i}=1\right)=P\left(\sigma_{i}=-1\right)=0.5$ and calculating the expectation over $\left\{\sigma_{i}\right\}$ :

$$
\mathcal{R}_{n}(\mathcal{F})=\underset{\sigma}{\mathbb{E}}\left[\sup _{f \in \mathcal{F}} \frac{1}{n} \sum_{i=1}^{n} \sigma_{i} f\left(\mathbf{x}_{i}\right)\right]
$$

Furthermore, we may take expectation over the samples $\mathbf{x}_{1}, \ldots, \mathbf{x}_{n}$ :

$$
\mathcal{R}(\mathcal{F})=\underset{\mathbf{x}_{i} \sim P_{x}}{\mathbb{E}} \underset{\sigma}{\mathbb{E}}\left[\sup _{f \in \mathcal{F}} \frac{1}{n} \sum_{i=1}^{n} \sigma_{i} f\left(\mathbf{x}_{i}\right)\right]
$$

Therefore, similar to $\mathrm{VC}$ dimension, the Rademacher average is high if the model class $\mathcal{F}$ is "rich" and can match most assignments of $\left\{\sigma_{i}\right\}$.

\section{Applications}

In many applications such as bioinformatics, the training examples are expensive and the number of features $p$ is much higher than the number of labeled examples $n$. In such cases, regularization is crucial, e.g., Zhang et al. (2008).

$L_{1}$ regularization has gained much popularity recently in the field of compressed sensing, and it has been widely used in imaging for radar, astronomy, medical diagnosis, and geophysics. See an ensemble of publications at http://dsp.rice. edu/cs.

The main spirit of regularization, namely, a preference for models with lower complexity, has been used by some $>$ model evaluation techniques. Examples include Akaike information criterion (AIC), Bayesian information criterion (BIC), minimum description length (MDL), and the minimum message length (MML).

\section{Cross-References}

- Minimum Description Length Principle

- Model Evaluation

> Occam's Razor

> Overfitting

- Support Vector Machines

- VC Dimension

\section{Recommended Reading}

Regularization lies at the heart of statistical machine learning, and it is indispensable in almost every learning algorithm. A comprehensive statistical analysis from the computational 
learning theory perspective can be found in Bousquet et al. (2005) and Vapnik (1998). Abundant resources on compressed sensing including both theory and applications are available at http://dsp.rice.edu/cs. Regularizations related to SVMs and kernel methods are discussed in detail by Schölkopf and Smola (2002) and Shawe-Taylor and Cristianini (2004). Anthony and Bartlett (1999) provide in-depth theoretical analysis for neural networks.

Anthony M, Bartlett PL (1999) Neural network learning: theoretical foundations. Cambridge University Press, Cambridge

Bousquet O, Boucheron S, Lugosi G (2005) Theory of classification: a survey of recent advances. ESAIM: Probab Stat 9:323-375

Candes E, Tao T (2005) Decoding by linear programming. IEEE Trans Inf Theory 51(12): 4203-4215

Devroye L, Györfi L, Lugosi G (1996) A probabilistic theory of pattern recognition. Applications of mathematics, vol 31. Springer, New York

Guo Y, Bartlett PL, Shawe-Taylor J, Williamson RC (1999) Covering numbers for support vector machines. In: Proceedings annual conference computational learning theory. Montreal, Canada

Kivinen J, Warmuth MK (1997) Exponentiated gradient versus gradient descent for linear predictors. Inf Comput 132(1):1-64

Rifkin RM, Lippert RA (2007) Value regularization and Fenchel duality. J Mach Learn Res 8: 441-479

Schölkopf B, Smola A (2002) Learning with kernels. MIT Press, Cambridge

Shawe-Taylor J, Cristianini N (2004) Kernel methods for pattern analysis. Cambridge University Press, Cambridge

Tibshirani R (1996) Regression shrinkage and selection via the LASSO. J R Stat Soc Ser B Stat Methodol 58:267-288

Tikhonov AN (1943) On the stability of inverse problems. Dokl Akad Nauk SSSR 39(5):195-198

Tropp JA (2006) Algorithms for simultaneous sparse approximation, Part II: convex relaxation. Signal Process 86(3):589C-602

Vapnik V (1998) Statistical learning theory. Wiley, New York

Vapnik V, Chervonenkis A (1971) On the uniform convergence of relative frequencies of events to their probabilities. Theory Probab Appl 16(2): 264-281

Zhang M, Zhang D, Wells MT (2008) Variable selection for large $p$ small $n$ regression models with incomplete data: mapping Qtl with epistases. BMC Bioinf 9:251

\section{Regularization Networks}

\author{
Radial Basis Function Networks
}

\section{Reinforcement Learning}

\section{Peter Stone}

Department of Computer Science, The

University of Texas at Austin, Austin, TX, USA

\section{Abstract}

This entry provides an overview of Reinforcement Learning (RL), with cross-references to specific RL algorithms.

Reinforcement learning describes a large class of learning problems characteristic of autonomous agents interacting in an environment: sequential decision-making problems with delayed reward. Reinforcement-learning algorithms seek to learn a policy (mapping from states to actions) that maximizes the reward received over time.

Unlike in $>$ supervised learning problems, in reinforcement-learning problems, there are no labeled examples of correct and incorrect behavior. However, unlike > unsupervised learning problems, a reward signal can be perceived.

Many different algorithms for solving reinforcement-learning problems are covered in other entries. This entry provides just a brief high-level classification of the algorithms.

Perhaps the most well-known approach to solving reinforcement-learning problems, as covered in detail by Sutton and Barto (1998), is based on learning a value function, which represents the long-term expected reward of each state the agent may encounter, given a particular policy. This approach typically assumes that the environment is a $>$ Markov decision process in which rewards are discounted over time, though it is also possible to optimize for average reward per time step as in $>$ average-reward reinforcement learning. If a complete model of the environment is available, $>$ dynamic programming, or specifically $>$ value iteration, 
can be used to compute an optimal value function, from which an optimal policy can be derived.

If a model is not available, an optimal value function can be learned from experience via model-free techniques such as $\triangleright$ temporal difference learning, which combine elements of dynamic programming with Monte Carlo estimation. Partly due to Watkins' elegant proof that $\triangleright$ Q-learning converges to the optimal value function (Watkins 1989), temporal difference methods are currently among the most commonly used approaches for reinforcement-learning problems.

Watkins' convergence proof relies on executing a policy that visits every state infinitely often. In practice, Q-learning does converge in small, discrete domains. However in larger and particularly in continuous domains, the learning algorithm must generalize the value function across states, a process known as $>$ value function approximation. Examples include $>$ instance-based reinforcement learning, $>$ Gaussian process reinforcement learning, and $>$ relational reinforcement learning.

Even when combined with value function approximation, the most basic value-free methods, such as Q-learning and SARSA, are very inefficient with respect to experience: they are not sample-efficient. With the view that experience is often more costly than computation, much research has been devoted to making more efficient use of experience, for instance, via $>$ hierarchical reinforcement learning, $>$ reward shaping, or $>$ model-based reinforcement learning in which the experience is used to learn a domain model, which can then be solved via dynamic programming.

Though these methods make efficient use of the experience that is presented to them, the goal of optimizing sample efficiency also motivates the study of $\triangleright$ efficient exploration in reinforcement learning. The study of exploration methods can be isolated from the full reinforcementlearning problem by removing the notion of temporally delayed reward as is done in $>$ associative reinforcement learning or by removing the notion of states altogether as is done in $>\mathrm{k}$-armed bandits. k-Armed bandit algorithms focus entirely on the exploration versus exploitation challenge, without having to worry about generalization across states or delayed rewards. Back in the context of the full RL problem, - Bayesian reinforcement learning enables optimal exploration given prior distributions over the parameters of the learning problem. However, its computational complexity has limited its use so far to very small domains.

Although most of the methods above revolve around learning a value function, reinforcementlearning problems can also be solved without learning value functions, by directly searching the space of potential policies via policy search. Effective ways of conducting such a search include $>$ policy gradient reinforcement learning, > least squares reinforcement-learning methods, and evolutionary reinforcement learning.

As typically formulated, the goal of a reinforcement-learning algorithm is to learn an optimal (or high-performing) policy based on knowledge of, or experience of, a reward function (and state transition function). However, it is also possible to take the opposite perspective that of trying to learn the reward function based on observation of the optimal policy. This problem formulation is known as $>$ inverse reinforcement learning.

Leveraging this large body of theory and algorithms, a current focus in the field is deploying large-scale, successful applications of reinforcement learning. Two such applications treated herein are autonomous helicopter flight using reinforcement learning and $>$ robot learning.

\section{Cross-References}
- Associative Reinforcement Learning
- Autonomous Helicopter Flight Using Rein- forcement Learning
- Average-Reward Reinforcement Learning
- Bayesian Reinforcement Learning
D Dynamic Programming
- Efficient Exploration in Reinforcement Learn- ing
- Gaussian Process Reinforcement Learning
- Hierarchical Reinforcement Learning 
- Instance-Based Reinforcement Learning

- Inverse Reinforcement Learning

> Least-Squares Reinforcement Learning Methods

- Model-Based Reinforcement Learning

$\checkmark$ Policy Gradient Methods

$\checkmark$ Q-Learning

- Relational Reinforcement Learning

- Reward Shaping

- Symbolic Dynamic Programming

- Temporal Difference Learning

- Value Function Approximation

\section{Recommended Reading}

Sutton RS, Barto AG (1998) Reinforcement learning: an introduction. MIT, Cambridge

Watkins CJCH (1989) Learning from delayed rewards. $\mathrm{PhD}$ thesis, King's College, Cambridge

\section{Reinforcement Learning in Structured Domains}

- Relational Reinforcement Learning

\section{Relational Data Mining}

- Inductive Logic Programming

\section{Relational Dynamic Programming}

- Symbolic Dynamic Programming

\section{Relational Learning}

Jan Struyf ${ }^{1}$ and Hendrik Blockeel ${ }^{1,2}$

${ }^{1}$ Katholieke Universiteit Leuven, Leuven,

Heverlee, Leuven, Belgium

${ }^{2}$ Leiden Institute of Advanced Computer

Science, Heverlee, Belgium

\section{Problem Definition}

Relational learning refers to learning in a context where there may be relationships between learn- ing examples, or where these examples may have a complex internal structure (i.e., consist of multiple components and there may be relationships between these components). In other words, the "relational" may refer to both an internal or external relational structure describing the examples. In fact, there is no essential difference between these two cases, as it depends on the definition of an example whether relations are internal or external to it. Most methods, however, are clearly set in one of these two contexts.

\section{Learning from Examples with External Relationships}

This setting considers learning from a set of examples where each example itself has a relatively simple description, for instance in the attributevalue format, and relationships may be present among these examples.

Example 1 Consider the task of web-page classification. Each web-page is described by a fixed set of attributes, such as a bag of words representation of the page. Web-pages may be related through hyperlinks, and the class label of a given page typically depends on the labels of the pages to which it links.

Example 2 Consider the Internet Movie Database (www.imdb.com). Each movie is described by a fixed set of attributes, such as its title and genre. Movies are related to other entity types, such as Studio, Director, Producer, and Actor, each of which is in turn described by a different set of attributes. Note that two movies can be related through the other entity types. For example, they can be made by the same studio or star the same well-known actor. The learning task in this domain could be, for instance, predicting the opening weekend box office receipts of the movies.

If relationships are present among examples, then the examples may not be independent and identically distributed (i.i.d.), an assumption made by many learning algorithms. Relational data that violates this assumption can be detrimental to learning performance as Jensen and Neville (2002) show. Relationships among 
examples can, on the other hand, also be exploited by the learning algorithm. $>$ Collective classification techniques (Jensen et al. 2004), for example, take the class labels of related examples into account when classifying a new instance.

\section{Learning from Examples with a Complex Internal Structure}

In this setting, each example may have a complex internal structure, but no relationships exist that relate different examples to one another. Learning algorithms typically use individualcentered representations in this setting, such as logical interpretations or strongly typed terms (Lloyd 2003), which store together all data of a given example. An important advantage of individual-centered representations is that they scale better to large datasets. Special cases of this setting include applications where the examples can be represented as graphs, trees, or sequences.

Example 3 Consider a database of candidate chemical compounds to be used in drugs. The molecular structure of each compound can be represented as a graph where the vertices are atoms and the edges are bonds. Each atom is labeled with its element type and the bonds can be single, double, triple, or aromatic bonds. Compounds are classified as active or inactive with regard to a given disease and the goal is to build models that are able to distinguish active from inactive compounds based on their molecular structure. Such models can, for instance, be used to gain insight in the common substructures, such as binding sites, that determine a compound's activity.

\section{Approaches to Relational Learning}

Many different kinds of learning tasks have been defined in relational learning, and an even larger number of approaches have been proposed for tackling these tasks. We give an overview of different learning settings that can be considered instances of relational learning.

\section{Inductive Logic Programming}

In $>$ inductive logic programming (ILP), the input and output knowledge of a learner are described in (variants of) first-order predicate logic. Languages based on first-order logic are highly expressive from the point of view of knowledge representation, and indeed, a language such as Prolog (Bratko 1986) can be used without adaptations to represent objects and the relationships between them, as well as background knowledge that one may have about the domain.

Example 4 This example is based on the work by Finn et al. (1998). Consider a data set that describes chemical compounds. The active compounds in the set are ACE inhibitors, which are used in treatments for hypertension. The molecular structure of the compounds is represented as a set of Prolog facts, such as: atom(m1, a1, o).

$\operatorname{atom}(\mathrm{m} 1, \mathrm{a} 2, \mathrm{c})$.

bond(m1, a1, a2, 1).

...

$\operatorname{coord}(\mathrm{m} 1, \mathrm{a} 1,5.91,-2.44,1.79)$.

$\operatorname{coord}(\mathrm{m} 1, \mathrm{a} 2,0.57,-2.77,0.33)$.

$\ldots$

which states that molecule $\mathrm{m} 1$ includes an oxygen atom a1 and a carbon atom a2 that are single bonded. The coord/5 predicate lists the $3 \mathrm{D}$ coordinates of the atoms in the given conformer. Background knowledge, such as the concepts zinc site, hydrogen donor, and the distance between atoms, are defined by means of Pro$\log$ clauses. Figure 1 shows a clause learned by the inductive logic programming system Progol (Džeroski and Lavraè 2001, Ch. 7) that makes use of these background knowledge predicates. This clause is the description of a pharmacophore, that is, a submolecular structure that causes a certain observable property of a molecule.

More details on the theory of inductive logic programming and descriptions of algorithms can be found in the entry on Inductive Logic Programming in this encyclopedia, or in references (De Raedt 2008; Džeroski and Lavraè 2001). 
a

ACE_inhibitor $(A):-$
$\operatorname{zincsite}(A, B)$,
$\operatorname{hacc}(A, C)$,
$\operatorname{dist}(A, B, C, 7.9,1.0)$,
$\operatorname{hacc}(A, D)$,
$\operatorname{dist}(A, B, D, 8.5,1.0)$,
$\operatorname{dist}(A, C, D, 2.1,1.0)$,
$\operatorname{hacc}(A, E)$,
$\operatorname{dist}(A, B, E, 4.9,1.0)$,
$\operatorname{dist}(A, C, E, 3.1,1.0)$,
$\operatorname{dist}(A, D, E, 3.8,1.0)$.

b

Molecule $A$ is an ACE inhibitor if:

molecule $A$ can bind to zinc at site $B$, and

molecule $A$ contains a hydrogen acceptor $C$, and

the distance between $B$ and $C$ is $7.9 \pm 1.0 \AA$, and

molecule $A$ contains a hydrogen acceptor $D$, and

the distance between $B$ and $D$ is $8.5 \pm 1.0 \AA$, and

the distance between $C$ and $D$ is $2.1 \pm 1.0 \AA$, and molecule $A$ contains a hydrogen acceptor $E$, and the distance between $B$ and $E$ is $4.9 \pm 1.0 \AA$, and the distance between $C$ and $E$ is $3.1 \pm 1.0 \AA$, and the distance between $D$ and $E$ is $3.8 \pm 1.0 \AA$.
Relational Learning, Fig. 1 (a) Prolog clause modeling the concept of an ACE inhibitor in terms of the background knowledge predicates zincsite/2, hacc/2, and dist/5. (b) The inductive logic programming system Pro- gol automatically translates (a) into the "Sternberg English" rule, which can be easily read by human experts. (c) $A$ molecule with the active site indicated by the atoms $B, C, D$, and $E$ (Image courtesy of Finn et al. 1998)

\section{Learning from Graphs}

A graph is a mathematical structure consisting of a set of nodes $V$ and a set of edges $E \subseteq V^{2}$ between those nodes. The set of edges is by definition a binary relation defined over the nodes. Hence, for any learning problem where the relationships between examples can be described using a single binary relation, the training set can be represented straightforwardly as a graph. This setting covers a wide range of relational learning tasks, for example, web mining (the set of links between pages is a binary relation), social network analysis, etc. Non-binary relationships can be represented as hypergraphs; in a hypergraph, edges are defined as subsets of $V$ of arbitrary size, rather than elements of $V^{2}$.

In graph-based learning systems, there is a clear distinction between approaches that learn from examples with external relationships, where the whole data set is represented as a single graph and each node is an example, and individualcentered approaches, where each example by itself is a graph. In the first kind of approaches, the goal is often to predict properties of existing nodes or edges, to predict the existence or nonexistence of edges ("link discovery"), to predict whether two nodes actually refer to the same object ("node identification"), detection of subgraphs that frequently occur in the graph, etc. When learning from multiple graphs, a typical goal is to learn a model for classifying the graphs, to find frequent substructures (where frequency is defined as the number of graphs a subgraphs occurs in), etc.

Compared to other methods for relational learning, graph-based methods typically focus more on the structure of the graph, and less on properties of single nodes. They may take node and edge labels into account, but typically do not allow for more elaborate information to be associated with each node.

- Graph mining methods are often more efficient than other relational mining methods because they avoid certain kinds of overhead, 
but are typically still NP-complete, as they generally rely on subgraph isomorphism testing. Nevertheless, researchers have been able to significantly improve efficiency or even avoid NP-completeness by looking only for linear or tree-shaped patterns, or by restricting the graphs analyzed to a relatively broad subclass. As an example, Horváth et al. (2006) show that a large majority of molecules belong to the class of outerplanar graphs, and propose an efficient algorithm for subgraph isomorphism testing in this class.

More information about mining graph data can be found in the - graph mining entry in this encyclopedia, or in Cook and Holder (2007) and Washio and Motoda (2003).

\section{Multi-relational Data Mining}

Multi-relational data mining approaches relational learning from the relational database point of view. The term "multi-relational" refers to the fact that from the database perspective, one learns from information spread over multiple tables or relations, as opposed to $>$ attribute-value learning, where one learns from a single table.

Multi-relational data mining systems tightly integrate with relational databases. Mainly rule and decision tree learners have been developed in this setting. Because practical relational databases may be huge, most of these systems pay much attention to efficiency and scalability, and use techniques such as sampling and precomputation (e.g., materializing views). An example of a scalable and efficient multirelational rule learning system is CrossMine (Yin et al. 2006).

An alternative approach to relational learning and multi-relational data mining is propositionalization. Propositionalization consists of automatically converting the relational representation into an attribute-value representation and then using attribute-value data mining algorithms on the resulting representation. An important line of research within multi-relational data mining investigates how database approaches can be used to this end. Database oriented propositionalization creates a view in which each example is represented by precisely one row. Information from related entities is incorporated into this row by adding derived attributes, computed by means of aggregation. In the movie database (Example 2), the view representing movies could include aggregated attributes such as the number of actors starring in the movie. A comparison of propositionalization approaches is presented by Krogel et al. (2003), and a discussion of them is also included in this volume.

Finally, note that most inductive logic programming systems are directly applicable to multi-relational data mining by representing each relational table as a predicate. This is possible because the relational representation is essentially a subset of first-order logic (known as datalog). Much research on multi-relational data mining was developed within the ILP community (Džeroski and Lavraè 2001).

\section{Statistical Relational \\ Learning/Probabilistic Logic Learning}

Research on relational learning, especially in the beginning, has largely focused on how to handle the relational structure of the data, and ignored aspects such as uncertainty. Indeed, the databases handled in multi-relational data mining, or the knowledge assumed given in inductive logic programming, are typically assumed to be deterministic. With the rise of probabilistic representations and algorithms within machine learning has come an increased interest in enabling relational learners to cope with uncertainty in the input data. This goal has been approached from at least two different directions: statistical learning approaches have been extended toward the relational setting, giving rise to the area of - statistical relational learning, whereas inductive logic programming researchers have investigated how to extend their knowledge representation and learning algorithms to cater for probabilistic information, referring to this research area as probabilistic logic learning. While there are some differences in terminology and approaches, both research areas essentially address the same research question, namely how to integrate relational and probabilistic learning.

Among the best known approaches for statistical relational learning is the learning of prob- 
abilistic relational models (PRMs, Džeroski and Lavraè 2001, Chap. 13). PRMs extend Bayesian networks to the relational representation used in relational databases. PRMs model the joint probability distribution over the non-key attributes in a relational database schema. Similar to Bayesian networks, PRMs are $>$ graphical models. Each attribute corresponds to a node and direct dependencies are modeled by directed edges. Such edges can connect attributes from different entity types that are (indirectly) related (such a relationship is called a "slot chain"). Inference in PRMs occurs by constructing a $>$ Bayesian network by instantiating the PRM with the data in the database and performing the inference in the latter. To handle 1:N relationships in the Bayesian network, PRMs make use of aggregation, similar to the propositionalization techniques mentioned above.

Bayesian logic programs (BLPs) (Kersting 2006) aim at combining the inference power of Bayesian networks with that of first-order logic reasoning. Similar to PRMs, the semantics of a BLP is defined by translating it to a Bayesian network. Using this network, the probability of a given interpretation or the probability that a given query yields a particular answer can be computed.

The acyclicity requirement of Bayesian networks carries over to representations such as PRMs and BLPs. Markov logic networks (MLNs) (Richardson and Domingos 2006) upgrade - Markov networks to first-order logic and allow networks with cycles. MLNs are defined as sets of weighted first-order logic formulas. These are viewed as "soft" constraints on logical interpretations: the fewer formulas a given interpretation violates, the higher its probability. The weight determines the contribution of a given formula: the higher its weight, the greater the difference in log probability between an interpretation that satisfies the formula and one that does not, other things being equal. The Alchemy system implements structure and parameter learning for MLNs.

More specific statistical learning techniques such as Naïve Bayes and Hidden Markov Models have also been upgraded to the relational setting. More information about such algorithms and about statistical relational learning in general can be found in Getoor and Taskar (2007) and Kersting (2006).

In probabilistic logic learning, two types of semantics are distinguished (De Raedt and Kersting 2003): the model theoretic semantics and the proof theoretic semantics. Approaches that are based on the model theoretic semantics define a probability distribution over interpretations and extend probabilistic attribute-value techniques, such as Bayesian networks and Markov networks, while proof theoretic semantics approaches define a probability distribution over proofs and upgrade, e.g., stochastic context free grammars.

Example 5 Consider the case where each example is a sentence in natural language. In this example, a model theoretic approach would define a probability distribution directly over sentences. A proof theoretic approach would define a probability distribution over "proofs," in this case possible parse trees of the sentence (each sentence may have several possible parses). Note that the proof theoretic view is more general in the sense that the distribution over sentences can be computed from the distribution over proofs.

Stochastic logic programs (SLPs) (Muggleton 1996) follow most closely the proof theoretic view and upgrade stochastic context free grammars to first-order logic. SLPs are logic programs with probabilities attached to the clauses such that the probabilities of clauses with the same head sum to 1.0. The probability of a proof is then computed as the product of the probabilities of the clauses that are used in the proof. PRISM (Sato and Kameya 1997) follows a related approach where the probabilities are defined on ground facts.

Like with standard graphical models, learning algorithms may include both parameter learning (estimating the probabilities) and structure learning (learning the program). For most frameworks mentioned above, such techniques have been or are being developed.

For a more detailed treatment of statistical relational learning and probabilistic logic learning, we refer to the entry on statistical relational learn- 
ing in this volume, and to several reference works (De Raedt and Kersting 2003; Getoor and Taskar 2007; Kersting 2006; De Raedt et al. 2008).

\section{Relational Reinforcement Learning}

Relational reinforcement learning (RRL) (Džeroski et al. 2001; Tadepalli et al. 2004) is reinforcement learning upgraded to the relational setting. Reinforcement learning is concerned with how an agent should act in a given environment to maximize its accumulated reward. In RRL, both the state of the environment and the actions are represented using a relational representation, typically in the form of a logic program.

Much research in RRL focuses on Q-learning, which represents the knowledge of the agent by means of a Q-function mapping state-action pairs to real values. During exploration, the agent selects in each state the action that is ranked highest by the Q-function. The Q-function is typically represented using a relational regression technique. Several techniques, such as relational regression trees, relational instance based learning, and relational kernel based regression have been considered in this context. Note that the regression algorithms must be able to learn incrementally: each time the agent receives a new reward, the Q-function must be incrementally updated for the episode (sequence of state-action pairs) that led to the reward. Due to the use of relational regression techniques, the agent is able to generalize over states: it will perform similar actions in similar states and therefore scales better to large application domains.

More recent topics in RRL include how expert knowledge can be provided to the agent in the form of guidance, and how learned knowledge can be transferred to related domains ("transfer learning"). More details on these techniques and more specific information on the topic of relational reinforcement learning can be found in its corresponding encyclopedia entry and in the related entry on $\triangleright$ symbolic dynamic programming, as well as in references Džeroski et al. (2001) and Tadepalli et al. (2004).

\section{Cross-References}

\author{
- Inductive Logic Programming \\ - Multi-relational Data Mining \\ - Relational Reinforcement Learning
}

\section{Recommended Reading}

Most of the topics covered in this entry have more detailed entries in this encyclopedia, namely "Inductive Logic Programming," "Graph Mining," "Relational Data Mining," and "Relational Reinforcement Learning." These entries provide a brief introduction to these more specific topics and appropriate references for further reading. Direct relevant references to the literature include the following. A comprehensive introduction to ILP can be found in De Raedt's book (De Raedt 2008) on logical and relational learning, or in the collection edited by Džeroski and Lavraè (2001) on relational data mining. Learning from graphs is covered by Cook and Holder (2007). Džeroski and Lavraè (2001) is also a good starting point for reading about multi-relational data mining, together with research papers on multi-relational data mining systems, for instance, Yin et al. (2006), who present a detailed description of the CrossMine system. Statistical relational learning in general is covered in the collection edited by Getoor and Taskar (2007), while De Raedt and Kersting (2003) and De Raedt et al. (2008) present overviews of approaches originating in logic-based learning. An overview of relational reinforcement learning can be found in Tadepalli et al. (2004).

Bratko I (2000) Prolog programming for artificial intelligence, 3rd edn. Addison-Wesley, Reading

Cook DJ, Holder LB (2007) Mining graph data. Wiley, Hoboken

De Raedt L (2008) Logical and relational learning. Springer, Berlin

De Raedt L, Kersting K (2003) Probabilistic logic learning. SIGKDD Explor 5(1):31-48

De Raedt L, Frasconi P, Kersting K, Muggleton S (2008) Probabilistic inductive logic programming. Springer, Berlin

Džeroski S, De Raedt L, Driessens K (2001) Relational reinforcement learning. Mach Learn 43:7-52

Džeroski S, Lavraè N (eds) (2001) Relational data mining. Springer, Berlin 
Finn P, Muggleton S, Page D, Srinivasan A (1998) Pharmacophore discovery using the inductive logic programming system PROGOL. Mach Learn 30:241-270

Getoor L, Taskar B (2007) Introduction to statistical relational learning. MIT Press, Cambridge

Horváth T, Ramon J, Wrobel S (2006) Frequent subgraph mining in outerplanar graphs. In: Proceedings of the 12th ACM SIGKDD international conference on knowledge discovery and data mining. ACM, New York, pp 197-206

Jensen D, Neville J (2002) Linkage and autocorrelation cause feature selection bias in relational learning. In: Proceeding of the 19th international conference on machine learning, University of New South Wales, Sydney. Morgan Kaufmann, San Francisco, pp 259-266

Jensen D, Neville J, Gallagher B (2004) Why collective inference improves relational classification. In: Proceedings of the 10th ACM SIGKDD international conference on knowledge discovery and data mining, Philadelphia. ACM, New York, pp 593-598

Kersting K (2006) An inductive logic programming approach to statistical relational learning. IOS Press, Amsterdam

Krogel M-A, Rawles S, Železný F, Flach P, Lavraè N, Wrobel S (2003) Comparative evaluation of approaches to propositionalization. In: Proceedings of the 13th international conference on inductive logic programming, Szeged. Springer, Berlin, pp 194-217

Lloyd JW (2003) Logic for learning. Springer, Berlin

Muggleton S (1996) Stochastic logic programs. In: De Raedt L (ed) Advances in inductive logic programming. IOS Press, Amsterdam, pp 254-264

Richardson M, Domingos P (2006) Markov logic networks. Mach Learn 62(1-2):107-136

Sato T, Kameya Y (1997) PRISM: a symbolicstatistical modeling language. In: Proceedings of the 15 th international joint conference on artificial intelligence (IJCAI 97), Nagoya. Morgan Kaufmann, San Francisco, pp 1330-1335

Tadepalli P, Givan R, Driessens K (2004) Relational reinforcement learning: an overview. In: Proceeding of the ICML'04 workshop on relational reinforcement learning, Banff, pp 1-9

Washio T, Motoda H (2003) State of the art of graphbased data mining. SIGKDD Explor 5(1):59-68

Yin X, Han J, Yang J, Yu PS (2006) Efficient classification across multiple database relations: a CrossMine approach. IEEE Trans Knowl Data Eng 18(6): 770-783

\section{Relational Regression Tree}

- First-Order Regression Tree

\section{Relational Reinforcement Learning}

Kurt Driessens

Maastricht University, Maastricht, The

Netherlands

\section{Synonyms}

Learning in worlds with objects; Reinforcement learning in structured domains

\section{Definition}

Relational reinforcement learning is concerned with learning behavior or control policies based on a numerical feedback signal, much like standard reinforcement learning, in complex domains where states (and actions) are largely characterized by the presence of objects, their properties, and the existing relations between those objects. Relational reinforcement learning uses approaches similar to those used for standard reinforcement learning, but extends these with methods that can abstract over specific object identities and exploit the structural information available in the environment.

\section{Motivation and Background}

- Reinforcement learning is a very attractive machine learning framework, as it tackles, in a sense, the whole artificial intelligence problem at a small scale: an agent acts in an unknown environment and has to learn how to behave optimally by reinforcement, i.e., through rewards and punishment. Reinforcement learning has produced some impressive and promising results. However, the applicability of reinforcement learning has been greatly limited by its difficulty in dealing with large problem spaces and its inability to generalize the learned knowledge to new but related problem domains. 
Relational

Reinforcement Learning,

Fig. 1 Structure of the

RRL system

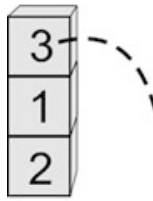

1
1
1
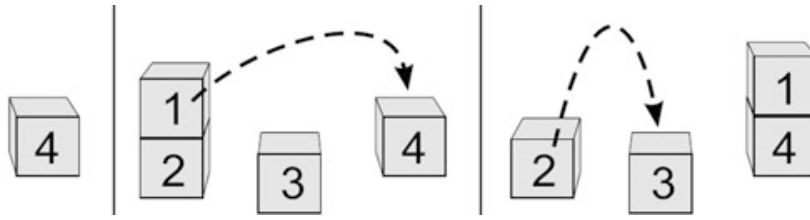

While standard reinforcement learning methods represent the learning environment as a set of unrelated states or, when using $>$ attribute-value representations, as a vector space consisting of a fixed number of independent dimensions, humans tend to think about their environment in terms of objects, their properties, and the relations between them. Examples of objects in everyday life are chairs, people, streets, trees, etc. This representation allows people to treat or use most of the new objects that they encounter correctly, without requiring training time to learn (again) how to use them. For example, people are able to drink their coffee from any cup that will hold it, even if they have never encountered that specific cup before, because they already have experience with drinking their coffee from other cup-type objects. Standard reinforcement learning agents do not have this ability. Their state and action representations do not allow them to abstract away from specific object identities and recognize them as a type of object they are already accustomed to.

Relational reinforcement learning tries to overcome this problem by representing states of the learning agent's environment as sets of objects, their properties, and the relationships between them, similar to the approaches used in relational learning and $>$ inductive logic programming. These structural representations make it possible for the relational reinforcement learning agent to abstract away from specific identities of objects and often also from the amount of objects present, the exact learning environment, or even the specific task to be performed.

The term "relational reinforcement learning" was introduced by Džeroski et al. (1998) when they first teamed the Q-learning algorithm with a first-order regression algorithm. From then on, relational reinforcement learning gained a large amount of interest.

\section{Structure of the Learning System}

In principle, the structure of a relational reinforcement learning system is very similar to that of standard reinforcement learning systems (Fig. 1). At a high level, the learning agent interacts with an environment by performing actions that influence that environment, and the environment provides the learning agent with a description of its current state and a numerical feedback of the performance of the agent. The goal of the agent is to maximize some cumulative form of this feedback signal. The major difference between standard reinforcement learning and relational reinforcement learning is the representation of the state-action-space. Relational reinforcement learning works on - Markov decision processes where states and actions have been relationally factored, so-called relational Markov decision processes (RMDPs).

An RMDP can be defined as follows:

Definition 1 (Relational Markov Decision Process) Let $P_{S}$ be a set of state-related predicates, $P_{A}$ a set of action-related predicates, and $C$ a set of constants in a logic $\Lambda$. Let $\mathcal{B}$ be a theory defined in that logic.

An RMDP is defined as $<S, A, T, R>$, where $S \equiv\left\{s \subset H^{P_{S} \cup C} \mid s \models \mathcal{B}\right\}$ represents the set of states; $A \equiv\left\{a \subset H^{P_{A} \cup C} \mid a \models \mathcal{B}\right\}$ represents the set of actions, in which $H^{X}$ is the set of facts that can be constructed given the symbols in $X$; and $T$ and $R$ represent the transition probabilities and reward function, respectively: $T: S \times A \times S \rightarrow[0,1]$ and $R: S \rightarrow \mathbb{R}$.

In less formal language, this means that the states and actions in an RMDP are represented using a set of constants $C$ and a set of predicates $P_{S}$ and $P_{A}$, respectively, and constrained by a background theory $\mathcal{B}$. This means that the background theory $\mathcal{B}$ defines which states are possible 


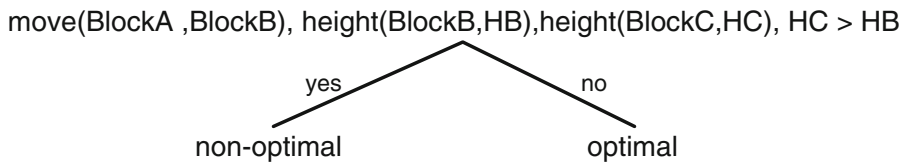

Relational Reinforcement Learning, Fig. 2 Example state-action pairs in the blocks world

in the domain and which actions can be executed in which states.

The following example illustrates these concepts. Consider the blocks world depicted in Fig. 2. To represent this environment in first-order logic, one could use:

- State-related predicates: $P_{S}=\{\mathrm{on} / 2$, clear $/ 1\}$

- Action-related predicate: $P_{A}=\{$ move $/ 2\}$

- Constants: $C=\{1,2,3,4$, floor $\}$

The set of facts $H^{P_{S} \cup C}$ would then include, for example, on (1,2), on $(4$, floor $)$, and clear (2) but also on $(3,3)$ and on ( floor, 2). To constrain the possible states to those that actually make sense in a standard, i.e., real-world view of the blocks world, the theory $\mathcal{B}$ can include rules to make states that include these kinds of facts impossible. For example, to make sure that a block cannot be on top of itself, $\mathcal{B}$ could include the following constraint:

$$
\text { false } \leftarrow \text { on }(X, X) .
$$

One can also include more extensive rules to define the exact physics of the blocks world that one is interested in. For example, by including

$$
\begin{gathered}
\text { false } \leftarrow \text { on }(Y, X), \text { on }(Z, X), X \neq \text { floor }, \\
Y \neq Z
\end{gathered}
$$

as part of the theory $\mathcal{B}$, one can exclude states where two blocks are on top of the same block. The action space given by $H^{P_{A} \cup C}$ consists of facts such as move $(3,2)$ and move ( floor, 1$)$ and can be constrained by rules such as

$$
\text { false } \leftarrow \text { move }(\text { floor }, X) \text {, }
$$

which makes sure that the floor cannot be placed on top of a block.
The leftmost state-action pair of Fig. 2 can be fully specified by the following set of facts (state description on the left, action on the right): on $(2$,floor $)$. clear(3). on $(1,2)$. clear(4). on $(3,1)$. clear(floor). on( 4 ,floor). move(3,floor).

One can easily generalize over specific states and create abstract states (or state-action pairs) that represent sets of states (or state-action pairs) by using variables instead of constants and by listing only those parts of states and actions that hold for each element of the abstract state (or state-action pair). For example, the abstract state "on $(1,2)$, on $(2$, floor $)$ " represents all states in which block 1 is on top of block 2, which in turn is on the floor. The abstract state does not specify the locations of any other blocks. Of the three states depicted in Fig. 2, the set of states represented by the abstract state would include the left and middle states. Abstract states can also be represented by using variables when one does not want to specify the location of any specific block, but wants to focus on structural aspects of the states and actions. The abstract state-action pair "move $(X, Y)$, on $(Y$, floor $)$ " represents all state-action pairs where a block is moved on top of another block that is on the floor, for example, the middle and right state-action pairs of Fig. 2.

\section{Benefits of Relational Reinforcement Learning}

We already stated that the real world is made of interacting objects or at least that humans often think about the real world as such. Relational reinforcement learning allows this same representation to be used by reinforcement learning agents, which in turn leads to more human-interpretable learning results. 


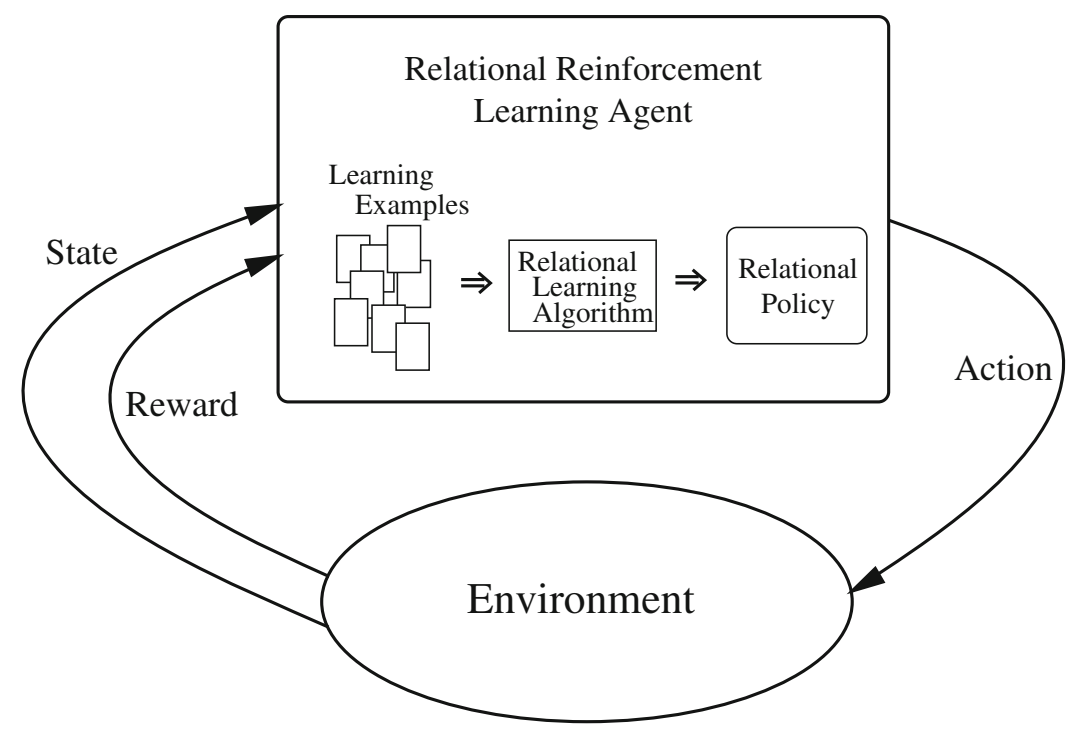

Relational Reinforcement Learning, Fig. 3 Simple relational policy for stacking any number of blocks

As a consequence of the used logical or relational representation of states and actions, the results learned by a relational reinforcement learning agent can be reused more easily when some of the parameters of the learning task change. Because relational reinforcement learning algorithms try to solve the problem at hand at an abstract level, the solutions will often carry over to different instantiations of that abstract problem. For example, the resulting policies learned by the RRL system (Driessens 2004) discussed below, a very simple example of which is shown in Fig. 3, often generalize over domains with a varying number of objects. If only actions which lead to the optimal leaf are executed, the shown policy tree will organize any number of blocks into a single stack.

As another example of this, the relational approximate policy iteration approach, also discussed below, is able to learn task-specific control knowledge from random walks in the environment. By treating the resulting state of such a random walk as a goal state and generalizing over the specifics of that goal (and the rest of the random walk), relational approximate policy iteration can learn domain-specific, but goalindependent, policies. This generalization of the policy is accomplished by parameterization of the goal and focusing on the relations between objects in the goal, states, and actions when representing the learned policy.

Another practical benefit of relational reinforcement learning lies in the field of inductive transfer. Transfer learning is concerned with the added benefits of having experience with a related task when being confronted with a new one. Because of the structural representation of learned results, the transfer of knowledge learned by relational reinforcement learning agents can be accomplished by recycling those parts of the results that still hold valid information for the new task. Depending on the relation between the two tasks, this can yield substantial benefits concerning the required training experience.

The use of first-order logic as a representational language in relational reinforcement learning also allows the integration of reasoning methods with traditional reinforcement learning approaches. One example of this is symbolic dynamic programming, which uses logical regression to compute necessary preconditions that allow an agent to reach certain goals. This same integration allows the use of search or planning knowledge as background information to extend the normal description of states and actions. 


\section{Example Relational Reinforcement Learning Approaches}

\section{Relational Q-Learning}

Relational reinforcement learning was introduced with the development of the RRL system (Džeroski et al. 1998). This is a Q-learning system that employs a relational regression algorithm to generalize the Q-table used by standard Q-learning algorithms into a Q-function. The differences with a standard Q-learning agent are mostly located inside the learning agent. One important difference is the agent's representation of the current state. In relational reinforcement learning, this representation contains structural or relational information about the environment.

Inside the learning agent, the information consisting of encountered states, chosen actions, and the associated rewards is translated into learning examples. These examples are then processed by a relational learning system that produces a relational Q-function and/or policy as a result. The relational representation of the Q-function allows the RRL system to use the structural properties of states and actions when assigning a Q-value to them.

Several relational regression approaches have been developed and applied in this context. While the original approach used an off-the-shelf relational regression algorithm that processed the learning examples in batch and had to be restarted to be able to process newly available learning experiences, a number of incremental algorithms have been developed for use in relational reinforcement learning since then. These include an incremental first-order regression tree algorithm, incremental relational instance-based regression, kernel-based regression that uses Gaussian processes, and graph kernels and algorithms that include combinations of the above (Driessens 2004).

It is possible to translate the learned Q-function approximations into a function that directly represents its policy. Using the values predicted by the learned Q-function, one can generate learning examples that represent state-action pairs and label them as either part of the learned policy or not. This results in a binary classification problem that can be handled by a supervised relational learning algorithm such as TILDE (Blockeel and De Raedt 1998), as used to produce first-order decision tree policies in the original work. This technique is known as P-learning. It exhibits better generalization performance across related learning problems than the Q-learning approach described above. Other than the aforementioned first-order decision trees, rule-based learners have also been applied to this kind of policy learning.

\section{Nonparametric Policy Gradients}

Nonparametric policy gradients (Kersting and Driessens 2008), also a model-free approach, apply Friedmann's gradient boosting (Friedman 2001) in an otherwise standard policy gradient approach for reinforcement learning. To avoid having to represent policies using a fixed number of parameters, policies are represented as a weighted sum of regression models grown in a stage-wise optimization. (This allows the number of parameters to grow as the experience of the learner increases, hence the name nonparametric.) While this does not make nonparametric policy gradients a technique specifically designed for relational reinforcement learning, it allows, like the relational Q-learning approach described above, the use of relational regression models and is not constrained to the attribute-value setting of standard policy gradients.

The idea behind the approach is that instead of finding a single, highly accurate policy, it is easier to find many rough rules of thumb of how to change the way the agent currently acts. The learned policy is represented as

$$
\pi(s, a)=\frac{e^{\Psi(s, a)}}{\sum_{b} e^{\Psi(s, b)}},
$$

where instead of assuming a linear parameterization for $\Psi$ as is done in standard policy gradients, it is assumed that $\Psi$ will be represented by a linear combination of functions. Specifically, one starts with some initial function $\Psi_{0}$, e.g., based on the zero potential, and iteratively adds corrections $\Psi_{m}=\Psi_{0}+\Delta_{1}+\cdots+\Delta_{m}$. In contrast to the standard gradient approach, $\Delta_{m}$ here denotes the 
so-called functional gradient, which is sampled during interaction with the environment and then generalized by an off-the-shelf regression algorithm.

The advantages of policy gradients over valuefunction techniques are that they can learn nondeterministic policies and that convergence of the learning process can be guaranteed, even when using function approximation (Sutton et al. 2000). Experimental results show that nonparametric policy gradients have the potential to significantly outperform relational Q-learning (Kersting and Driessens 2008).

\section{Relational Approximate Policy Iteration}

A different approach, which also directly learns a policy, is taken in relational approximate policy iteration (Fern et al. 2006). Like standard policy iteration (Sutton and Barto 1998), the approach iteratively improves its policy through interleaving evaluation and improvement steps. In contrast to standard policy iteration, it uses a policy language bias and a generalizing policy function.

Instead of building a value-function approximation for each policy evaluation step, relational approximate policy iteration evaluates the current policy and its closely related neighbors by sampling the state-action-space through a technique called policy rollout. This technique generates a set of trajectories from a given state, by executing every possible action in that state and following the current policy for a number of steps afterward. (It is also possible to improve convergence speed by following the next policy.) These trajectories and their associated costs result in a number of learning examples - one for each possible action in each selected state - that can be used, together with the policy language bias to generate the next, improved policy.

Because every possible action in each sampled state needs to be evaluated, this approach does require a model or a resettable simulator of the environment. However, relational approximate policy iteration has been shown to work well for learning domain-specific control knowledge and performs very well on planning competition problems.
Relational Cross Entropy Policy Search

The most recent addition to direct relational policy search uses the cross entropy method to evaluate, select, expand, and combine those pieces of a modular policy that lead to high-performance behavior (Sarjant et al. 2014). The policy pieces are singular condition-action rules. The rules are constructed and adapted automatically using a partial model of the environment inferred from interactions with that environment. The model defines the minimal conditions needed to take an action, the possible specialization conditions per rule, and a set of simplification rules to remove redundant and illegal rule conditions. Rule construction and specialization follow a principled approach toward exploration of the rule space by beginning with the relative least general generalization (RLGG) rules and then exploring incremental specializations of interesting rules.

The cross entropy method (CEM) (Rubinstein 1997 ) is used to find these interesting rules. CEM tunes the selection probabilities of the rules according to the performance of policies that they participate in. Rules with high selection probabilities also become candidates for specialization, possibly giving rise to even better rules.

The resulting systems can learn behavior that is competitive to specialized approaches on complex tasks, while the built-in simplification of the rules and CEM bias toward compact policies result in comprehensive and effective relational policies.

\section{Symbolic Dynamic Programming}

In contrast to the previous techniques, $\triangleright$ symbolic dynamic programming (SDP) does not learn a policy through exploration of the environment. Instead, it is a model-based approach that uses knowledge about preconditions and consequences of actions to compute the fastest way to reach a given goal. Like other dynamic programming techniques, SDP starts from the goal the agent wants to reach and reasons backward to find the policy that is needed to reach that goal. In contrast to other dynamic programming techniques, it does not solve specific instantiations of the problem domain, but instead solves the problem at an abstract level, 
thereby solving it for all possible instantiations of the problem at once.

SDP treats the required goal conditions as an abstract state definition. Because pre- and postconditions of actions are known, SDP can compute the necessary conditions that allow actions to reach the abstract goal state. These conditions define abstract states from which it is possible to reach a goal state in one step. Starting from these abstract states, the same approach can be used to discover abstract states that allow the goal to be reached in two steps and so on.

This approach was first proposed by Boutilier et al. (2001), implemented as a working system by Kersting et al. (2004), and later improved upon by Sanner and Boutilier (2005). This last approach won second place in the probabilistic programming competition at ICAPS in 2006.

\section{Cross-References}

\author{
- Hierarchical Reinforcement Learning \\ - Inductive Logic Programming \\ - Model-Based Reinforcement Learning \\ $\checkmark$ Policy Search \\ Q-Learning \\ $\checkmark$ Reinforcement Learning \\ - Relational Learning \\ - Symbolic Dynamic Programming \\ - Temporal Difference Learning
}

\section{Further Information}

The field of relational reinforcement learning has given rise to a number of $\mathrm{PhD}$ dissertations in the last few years (Croonenborghs 2009; Driessens 2004; van Otterlo 2008; Sanner 2008). The dissertation of Martijn van Otterlo resulted in a book (van Otterlo 2009) which provides a recent and reasonably complete overview of the relational reinforcement learning research field. Other publications that present an overview of relational reinforcement learning research include the proceedings of the two workshops on representational issues in (relational) reinforcement learning at the International Conferences on Machine
Learning in 2004 and 2005 (Driessens et al. 2005; Tadepalli et al. 2004).

\section{Recommended Reading}

Blockeel H, De Raedt L (1998) Top-down induction of first order logical decision trees. Artif Intell 101(12):285-297

Boutilier C, Reiter R, Price B (2001) Symbolic dynamic programming for first-order MDPs. In: Proceedings of the 17th international joint conference on artificial intelligence (IJCAI-2001), Seattle, pp 690-700

Croonenborghs T (2009) Model-assisted approaches for relational reinforcement learning. Ph.D. thesis, Department of Compute Science, Katholieke Universiteit Leuven

Driessens K (2004) Relational reinforcement learning. Ph.D. thesis, Department of Computer Science, Katholieke Universiteit Leuven

Driessens K, Fern A, van Otterlo M (eds) (2005) Proceedings of ICML-2005 workshop on rich representation for reinforcement learning, Bonn

Džeroski S, De Raedt L, Blockeel H (1998) Relational reinforcement learning. In: Proceedings of the 15th international conference on machine learning (ICML-1998), San Francisco. Morgan Kaufmann, Madison, pp 136-143

Džeroski S, De Raedt L, Driessens K (2001) Relational reinforcement learning. Mach Learn 43:7-52

Fern A, Yoon S, Givan R (2006) Approximate policy iteration with a policy language bias: solving relational Markov decision processes. J Artif Intell Res 25:85-118

Friedman J (2001) Greedy function approximation: a gradient boosting machine. Ann Stat 29:1189-1232

Kersting K, Driessens K (2008) Non-parametric policy gradients: a unified treatment of propositional and relational domains. In: McAllum A, Roweis S (eds) Proceedings of the 25th international conference on machine learning (ICML 2008), Helsinki, pp 456-463

Kersting K, van Otterlo M, De Raedt L (2004) Bellman goes relational. In: Proceedings of the twentyfirst international conference on machine learning (ICML-2004), Banff, pp 465-472

Rubinstein RY (1997) Optimization of computer simulation models with rare events. Eur J Oper Res 99(1):89-112

Sanner S (2008) First-order decision-theoretic planning in structured relational environments. Ph.D. thesis, Department of Compute Science, University of Toronto

Sanner S, Boutilier C (2005) Approximate linear programming for first-order MDPs. In: Proceedings of the 21 st conference on Uncertainty in AI (UAI), Edinburgh 
Sarjant S (2013) Policy search based relational reinforcement learning using the cross-entropy method. Ph.D. thesis, Department of Computer Science, University of Waikato

Sarjant S, Pfahringer B, Driessens K, Smith T (2014) A Direct Policy-Search Algorithm for Relational Reinforcement Learning. In: Proceedings of the 25th international conference on inductive logic programming (ILP 2013), Rio de Janeiro, pp 76-92

Sutton RS, Barto AG (1998) Reinforcement learning: an introduction. MIT, Cambridge

Sutton RS, McAllester D, Singh S, Mansour Y (2000) Policy gradient methods for reinforcement learning with function approximation. In: Advances in neural information processing systems, vol 12. MIT, Cambridge, pp 1057-1063

Tadepalli P, Givan R, Driessens K (eds) (2004) Proceedings of the ICML-2004 workshop on relational reinforcement learning, Banff

van Otterlo M (2008) The logic of adaptive learning. $\mathrm{Ph} . \mathrm{D}$. thesis, Centre for Telematics and Information Technology, University of Twente

van Otterlo M (2009) The logic of adaptive behavior: knowledge representation and algorithms for adaptive sequential decision making under uncertainty in first-order and relational domains. IOS Press, Amsterdam

\section{Relational Value Iteration}

- Symbolic Dynamic Programming

\section{Relationship Extraction}

$>$ Link Prediction

\section{Relevance Feedback}

Relevance feedback provides a measure of the extent to which the results of a search match the expectations of the user who initiated the query. Explicit feedback require users to assess relevance by choosing one out of a number of choices, or to rank documents to reflect their perceived degree of relevance. Implicit feedback is obtained by monitoring user's behavior such as time spent browsing a document, amount of scrolling performed while browsing a document, number of times a document is visited, etc. Relevance feedback is one the techniques used to support query reformulation and turn the search into an iterative and interactive process.

\section{Cross-References}

- Search Engines: Applications of ML

\section{Representation Language}

Hypothesis Language

\section{Reservoir Computing}

Risto Miikkulainen

Department of Computer Science, The

University of Texas at Austin, Austin, TX, USA

\section{Synonyms}

Echo state network; Liquid state machine

\section{Definition}

Reservoir computing is an approach to sequential processing where recurrency is separated from the output mapping (Jaeger 2003; Maass et al. 2002). The input sequence activates neurons in a recurrent neural network (a reservoir, where activity propagates as in a liquid). The recurrent network is large, nonlinear, randomly connected, and fixed. A linear output network receives activation from the recurrent network and generates the output of the entire machine. The idea is that if the recurrent network is large and complex enough, the desired outputs can likely be learned as linear transformations of its activation. Moreover, because the output transformation is linear, it is fast to train. Reservoir computing has been successful in particular in speech and language processing and vision and cognitive neuroscience. 


\section{Recommended Reading}

Jaeger H (2003) Adaptive nonlinear system identification with echo state networks. In: Becker S, Thrun $\mathrm{S}$, Obermayer K (eds) Advances in neural information processing systems, vol 15. MIT, Cambridge, pp 593-600

Maass W, Natschlaeger T, Markram H (2002) Realtime computing without stable states: a new framework for neural computation based on perturbations. Neural Comput 14:2531-2560

\section{Resubstitution Estimate}

Resubstitution estimates are estimates that are derived by applying a model to the $>$ training data from which it was learned. For example, resubstitution error is the error of a model on the training data.

\section{Cross-References}

- Model Evaluation

\section{Reward}

In most Markov decision process applications, the decision-maker receives a reward each period. This reward can depend on the current state, the action taken, and the next state and is denoted by $r_{t}\left(s, a, s^{\prime}\right)$.

\section{Reward Selection}

$>$ Reward Shaping

\section{Reward Shaping}

Eric Wiewiora

University of California, Sydney, NSW, Australia

\section{Synonyms}

Heuristic rewards; Reward selection

\section{Definition}

Reward shaping is a technique inspired by animal training where supplemental rewards are provided to make a problem easier to learn. There is usually an obvious natural reward for any problem. For games, this is usually a win or loss. For financial problems, the reward is usually profit. Reward shaping augments the natural reward signal by adding additional rewards for making progress toward a good solution.

\section{Motivation and Background}

Reward shaping is a method for engineering a reward function in order to provide more frequent feedback on appropriate behaviors. It is most often discussed in the $>$ reinforcement learning framework. Providing feedback is crucial during early learning so that promising behaviors are tried early. This is necessary in large domains, where reinforcement signals may be few and far between.

A good example of such a problem is chess. The objective of chess is to win a match, and an appropriate reinforcement signal should be based on this. If an agent were to learn chess without prior knowledge, it would have to search for a great deal of time before stumbling onto a winning strategy. We can speed up this process by rewarding the agent more frequently. One possibility is to reward the learner for capturing enemy pieces, and punish the learner for losing pieces. This new reward creates a much richer learning environment, but also runs the risk of distracting the agent from the true goal (winning the game).

Another domain where feedback is extremely important is in robotics and other real-world applications. In the real world, learning requires a large amount of interaction time, and may be quite expensive. Mataric noted that in order to mitigate "thrashing" (repeatedly trying ineffective actions) rewards should be supplied as often as possible (Mataric 1994).

If a problem is inherently described by sparse rewards, it may be difficult to change the re- 
ward structure without disrupting progress to the original goal. The behavior that is optimal with a richer reward function may be quite different from the intended behavior, even if relatively small shaping rewards are added. A classic example of this is found in Randlov and Alstrom (1998). While training an agent to control a bicycle simulation, they rewarded an agent whenever it moved toward a target destination. In response to this reward, the agent learned to ride in a tight circle, receiving reward whenever it moved in the direction of the goal.

\section{Theory}

We assume a reinforcement learning framework. For every time step $t$, the learner observes state $s_{t}$, takes action $a_{t}$, and receives reward $r_{t}$. The goal of reinforcement learning is to find a policy $\pi(s)$ that produces actions that optimize some longterm measurement of reward. We define the value function for every state as the expected infinite horizon discounted reward

$$
V(s)=\max _{\pi} \mathrm{E}\left[\sum_{t=0}^{\infty} \gamma^{t} r_{t} \mid s_{0}=s, a_{t}=\pi\left(s_{t}\right)\right],
$$

where $\gamma$ is the discount rate. A reinforcement learner's goal is to learn a good estimate of $V(s)$, and to use this estimate to choose a good policy.

A natural reward source should be fairly obvious from the problem at hand. Financial problems should use net monetary gain or loss as reward. Games and goal-directed problems should reward winning the game or reaching the goal. It is usually advantageous to augment this natural reward with a shaping reward $f_{t}$. We define the augmented value function $V^{\prime}$ for the reinforcement learning problem with shaping rewards

$$
\begin{aligned}
V^{\prime}(s) & =\max _{\pi^{\prime}} \mathrm{E}\left[\sum_{t=0}^{\infty} \gamma^{t}\left(r_{t}+f_{t}\right) \mid s_{0}\right. \\
& \left.=s, a_{t}=\pi^{\prime}\left(s_{t}\right)\right] .
\end{aligned}
$$

Ideally, the policy that optimizes the augmented value function will differ much from the previous optimal policy.

Constructing an appropriate shaping reward system is inherently a problem-dependent task, though a line of research aids in the implementation of these reward signals. Potentialbased shaping provides a formal framework for translating imperfect knowledge of the relative value of states and actions into a shaping reward.

\section{Potential-Based Shaping}

$\mathrm{Ng}$ et al. proposed a method for adding shaping rewards in a way that guarantees the optimal policy maintains its optimality ( $\mathrm{Ng}$ et al. 1999). They define a potential function $\Phi()$ over the states. The shaping reward $f$ for transitioning from state $s$ to $s^{\prime}$ is defined as the discounted change in this state potential:

$$
f\left(s, s^{\prime}\right)=\gamma \Phi\left(s^{\prime}\right)-\Phi(s) .
$$

This potential-based shaping reward is added to the natural reward for every state transition the learner experiences. Call the augmented reward $r_{t}^{\prime}=r_{t}+f\left(s_{t}, s_{t+1}\right)$, and the value function based on this reward $V^{\prime}(s)$. The potential-based shaping concept can also be applied to actions as well as states. See Wiewiora et al. (2003) for details.

It can be shown that the augmented value function is closely related to the original:

$$
V^{\prime}(s)=V(s)-\Phi(s) .
$$

An obvious choice for the potential function is $\Phi(s) \approx V(s)$, making $V^{\prime}()$ close to zero. This intuition is strengthened by results presented by Wiewiora (2003). This paper shows that for most reinforcement learning systems, the potential function acts as an initial estimate of the natural value function $V()$. 
However, even if the potential function used for shaping is very close to the true natural value function, learning may still be difficult. Koenig et al. have shown that initial estimates of the value function have a large influence on the efficiency of reinforcement learning (Koenig and Simmons 1996). With an initial estimate of the value function set below the optimal value, many reinforcement learning algorithms could require learning time exponential in the state and action space in order to find a highly rewarding state. On the other hand, in nonrandom environments, an optimistic initialization the value function creates learning time that is polynomial in the stateaction space before a goal is found.

\section{Cross-References}

> Reinforcement Learning

\section{Recommended Reading}

Koenig S, Simmons RG (1996) The effect of representation and knowledge on goal directed exploration with reinforcement-learning algorithms. Mach Learn 22(1-3):227-250

Mataric MJ (1994) Reward functions for accelerated learning. In: International conference on machine learning, New Brunswick. Morgan Kaufmann, San Francisco, pp 181-189

Ng AY, Harada D, Russell S (1999) Policy invariance under reward transformations: theory and application to reward shaping. In: Machine learning, proceedings of the sixteenth international conference, Bled. Morgan Kaufmann, San Francisco, pp 278-287

Randlov J, Alstrom P (1998) Learning to drive a bicycle using reinforcement learning and shaping. In: Proceedings of the fifteenth international conference on machine learning, Madison. Morgan Kaufmann, San Francisco

Wiewiora E (2003) Potential-based shaping and Qvalue initialization are equivalent. J Artif Intell Res 19: 205-208

Wiewiora E, Cottrell G, Elkan C (2003) Principled methods for advising reinforcement learning agents. In: Machine learning, proceedings of the twentieth international conference, Washington, DC. AAAI Press, Menlo Park, pp 792-799

\section{Robot Learning}

Jan Peters ${ }^{1,2,3}$, Russ Tedrake ${ }^{4}$, Nick Roy ${ }^{4}$, and Jun Morimoto ${ }^{5}$

${ }^{1}$ Max Planck Institute for Biological

Cybernetics, Tübingen, Germany

${ }^{2}$ Intelligent Autonomous Systems, Computer

Science Department, Technische Universität

Darmstadt, Darmstadt, Hessen, Germany

${ }^{3}$ Department of Empirical Inference,

Max-Planck Institute for Intelligent Systems,

Tübingen, Germany

${ }^{4}$ Massachusetts Institute of Technology,

Cambridge, MA, USA

${ }^{5}$ Advanced Telecommunication Research

Institute International (ATR), Kyoto, Japan

\section{Definition}

- Robot learning consists of a multitude of machine learning approaches, particularly $>$ reinforcement learning, $>$ inverse reinforcement learning, and $>$ regression methods, that have been adapted sufficiently to domain so that they allow learning in complex robot systems such as helicopters, flapping-wing flight, legged robots, anthropomorphic arms, and humanoid robots. While classical artificial intelligencebased robotics approaches have often attempted to manually generate a set of rules and models that allows the robot systems to sense and act in the real world, $>$ robot learning centers around the idea that it is unlikely that we can foresee all interesting real-world situations sufficiently accurate. Hence, the field of $>$ robot learning assumes that future robots need to be able to adapt to the real world, and domain-appropriate machine learning might offer the most approach in this direction.

\section{Robot Learning Systems}

As learning has found many backdoor entrances to robotics, this section can only scratch the surface. However, robot learning has clearly been 
successful in several areas: (i) model learning, (ii) imitation and apprenticeship learning, and (iii) reinforcement learning as well as in various other topics.

\section{Model Learning}

Model learning is the machine learning counterpart to classical system identification (Farrell and Polycarpou 2006; Schaal et al. 2002). However, while the classical approaches heavily rely on the structure of physically based models, specification of the relevant state variables, and handtuned approximations of unknown nonlinearities, model learning approaches avoid many of these labor-intensive steps and the entire process to be more easily automated. Machine learning and system identification approaches often assume an observable state of the system to estimate the mapping from inputs to outputs of the system. However, a learning system is often able to learn this mapping including the statistics needed to cope with unidentified state variables and can hence cope with a larger class of systems. Two types of models are commonly learned, i.e., forward and inverse models.

Forward models predict the behavior of the system based either on the current state or a history of preceding observations. They can be viewed as "learned simulators" that may be used for optimizing a policy or for predicting future information. Examples of the application of such learned simulators range from the early work in the late 1980s by Atkeson and Schaal in robot arm-based cart pole swing-ups to $\mathrm{Ng}$ 's recent extensions for stabilizing an inverted helicopter. Most forward models can directly be learned by $>$ regression.

Conversely, inverse models attempt to predict the input to a system in order to achieve a desired output in the next step, i.e., it uses the model of the system to directly generate control signals. In traditional control, these are often called approximation-based control systems (Farrell and Polycarpou 2006). Inverse model learning can be straightforwardly by - regression when the system dynamics can be inverted uniquely, e.g., as in inverse dynamic learning for a fully actuated system. However, for underactuated or redundantly actuated systems (Tedrake 2009), operational space control (Peters and Schaal 2008a), etc., such unique inverses do not exist and additional optimization is needed.

\section{Imitation and Apprenticeship Learning}

A key problem in robotics is to ease the problem of programming a complex behavior. Traditional robot programming approaches rely on accurate, manual modeling of the task and removal of all uncertainties so that they work well. In contrast to classical robot programming, learning from demonstration approaches aims at recovering the instructions directly from a human demonstration. Numerous unsolved problems exist in this context such as discovering the intent of the teacher or determining the mapping from the teacher's kinematics to the robot's kinematics (often called the correspondence problem). Two different approaches are common in this area, i.e., direct imitation learning and apprenticeship learning.

In imitation learning (Schaal et al. 2003), also known as $>$ behavioral cloning, the robot system directly estimates a policy from a teachers presentation, and, subsequently, the robot system reproduces the task using this policy. A key advantage of this approach is that it can often learn a task successfully from few demonstrations. In areas where human demonstrations are straightforward to obtain, e.g., for learning racket sports, manipulation, drumming on anthropomorphic systems, direct imitation learning often proved to be an appropriate approach. Its major shortcomings are that it cannot explain why the derived policy is a good one, and it may struggle with learning from noisy demonstrations.

Hence, apprenticeship learning (Coates et al. 2009) has been proposed as an alternative where a reward function is used as explanation of the teachers' behavior. Here, the reward function is chosen under which the teacher appears to act optimally, and the optimal policy for this reward 
function is subsequently computed as a solution. This approach transforms the problem of learning from demonstrations onto the harder problem of approximate optimal control or reinforcement learning; hence it is also known as inverse optimal control or $>$ inverse reinforcement learning. As a result, it is limited to problems that can be solved by current reinforcement learning methods. Additionally, it often has a hard time dealing with tasks where only few demonstrations with low variance exist. Hence, inverse reinforcement learning has been particularly successful in areas where it is hard for a human to demonstrate the desired behavior such as for helicopter acrobatics or in robot locomotion.

Further information on learning by demonstration may be found in Coates et al. (2009) and Schaal et al. (2003).

\section{Robot Reinforcement Learning}

The ability to self-improve with respect to an arbitrary reward function, i.e., $>$ reinforcement learning, is essential for robot systems to become more autonomous. Here, the system learns about its policy by interacting with its environment and receiving scores (i.e., rewards or costs) for the quality of its performance. Unlike supervised learning approaches used in model learning or imitation learning, reinforcement learning can still be considered to be in its infancy. Few offthe-shelf reinforcement learning methods scale into the domain of robotics both in terms of dimensionality and the number of trials needed to obtain an interesting behavior. Three different but overlapping styles of reinforcement learning can be found in robotics, i.e., model-based reinforcement learning, - value function approximation methods, and direct $>$ policy search.

Model-based reinforcement learning relies upon a learned forward model used for simulation-based optimization as discussed before. While often highly efficient, it frequently suffers from the fact that learned models are imperfect, and hence, the optimization method can be guaranteed to be biased by the errors in the model. To date, a full Bayesian treatment of the model uncertainty appears to be a promising way for alleviating this shortcoming of this otherwise powerful approach.

Value function approximation methods have been the core approach used in reinforcement learning during the 1990s. These techniques rely upon approximating the expected rewards for every possible action in every visited state. Subsequently, the controller chooses the actions in accordance to this value. Such approximation requires a globally consistent value function where the quality of the policy is determined by the largest error of the value function at any possible state. As a result, these methods have been problematic for anthropomorphic robotics as the highdimensional domains often defy learning such a global construct. However, it has been highly successful in low-dimensional domains such as mobile vehicle control and robot soccer, as well as on well-understood test domains such as cartpole systems.

Unlike the previous two approaches, policy search attempts to directly learn the optimal policy from experience without solving intermediary learning problems. Policies often have significantly fewer parameters than models or value functions. For example, for balancing a ball on a plate (where the plate is mounted on a robot end effector) optimally with respect to a quadratic reward function, the number of policy parameters grows linearly in the number state dimensions, while it grows quadratically for both model and value function for this analytically tractable problem (in general cases, the number of parameters of value functions grows exponentially in the number of states which is known as the "curse of dimensionality"). This insight has given rise to policy search methods, particularly, > policy gradient methods and probabilistic approaches to policy search such as the reward-weighted regression or PoWER. To date, application results of direct policy search approaches range from gait optimization in locomotion to various motor learning examples (e.g., Kendama, T-Ball, or throwing darts).

Further information on reinforcement learning for robotics may be found in Tedrake et al. (2004), Peters and Schaal (2008b), and Riedmiller et al. (2009). 


\section{Application Domains}

The possible application domains for robot learning have not been fully explored, one could even aggressively state that we have barely started to bring learning into robotics. Nevertheless, robot learning has been successful in several application domains.

For accurate execution of desired trajectories, model learning has scaled to learning the full inverse dynamics for a humanoid robot in real time more accurately than achievable with physical models. Current work focusses mainly on improving the concurrent execution of tasks as well as control of redundant or underactuated systems.

Various approaches have been successful in task learning. Learning by demonstration approaches is moving increasingly toward industrial grade solutions where fast training of complex tasks becomes possible. Skills ranging from motor toys, e.g., basic movements, paddling a ball, etc., to complex tasks such as cooking a complete meal, basic table tennis strokes, helicopter acrobatics, or foot placement in locomotion have been learned from human teachers. Reinforcement learning has yielded better gaits in locomotion, jumping behaviors for legged robots, perching with fixed wing flight robots, forehands in table tennis, as well as various applications to learning of motor toys.

\section{Cross-References}

- Behavioral Cloning

- Inverse Reinforcement Learning

$\checkmark$ Policy Search

- Reinforcement Learning

- Value Function Approximation

\section{Recommended Reading}

Coates A, Abbeel P, Ng AY (2009) Apprenticeship learning for helicopter control. Commun ACM 52(7):97-105

Farrell JA, Polycarpou MM (2006) Adaptive approximation based control. Adaptive and learning systems for signal processing, communications and control series. Wiley, Hoboken
Peters J, Schaal S (2008a) Learning to control in operational space. Int J Robot Res 27:197-212

Peters J, Schaal S (2008b) Reinforcement learning of motor skills with policy gradients. Neural Netw 21(4):682-697

Riedmiller M, Gabel T, Hafner R, Lange S (2009) Reinforcement learning for robot soccer. Auton Robot 27(1):55-73

Schaal S, Atkeson CG, Vijayakumar S (2002) Scalable techniques from nonparameteric statistics for realtime robot learning. Appl Intell 17(1):49-60

Schaal S, Ijspeert A, Billard A (2003) Computational approaches to motor learning by imitation. Philos Trans R Soc Lond: Ser B Biol Sci 358(1431): 537-547

Tedrake R (2009) Underactuated robotics: learning, planning, and control for efficient and agile machines. Course notes for MIT 6.832, MIT 32-380, Cambridge

Tedrake R, Zhang TW, Seung HS (2004) Stochastic policy gradient reinforcement learning on a simple $3 \mathrm{~d}$ biped. In: Proceedings of the IEEE international conference on intelligent robots and systems (IROS), Sendai, pp 2849-2854

\section{ROC Analysis}

Peter A. Flach

Department of Computer Science, University of Bristol, Bristol, UK

\section{Synonyms}

Receiver operating characteristic analysis

\section{Definition}

ROC analysis investigates and employs the relationship between $>$ sensitivity and $>$ specificity of a binary classifier. Sensitivity or $>$ true positive rate measures the proportion of positives correctly classified; specificity or $>$ true negative rate measures the proportion of negatives correctly classified. Conventionally, the true positive rate $t p r$ is plotted against the false positive rate $f p r$, which is one minus true negative rate. If a classifier outputs a score proportional to its belief that an instance belongs to the positive class, decreasing the $>$ decision threshold above which an instance is deemed to belong to the positive class - will increase both true 


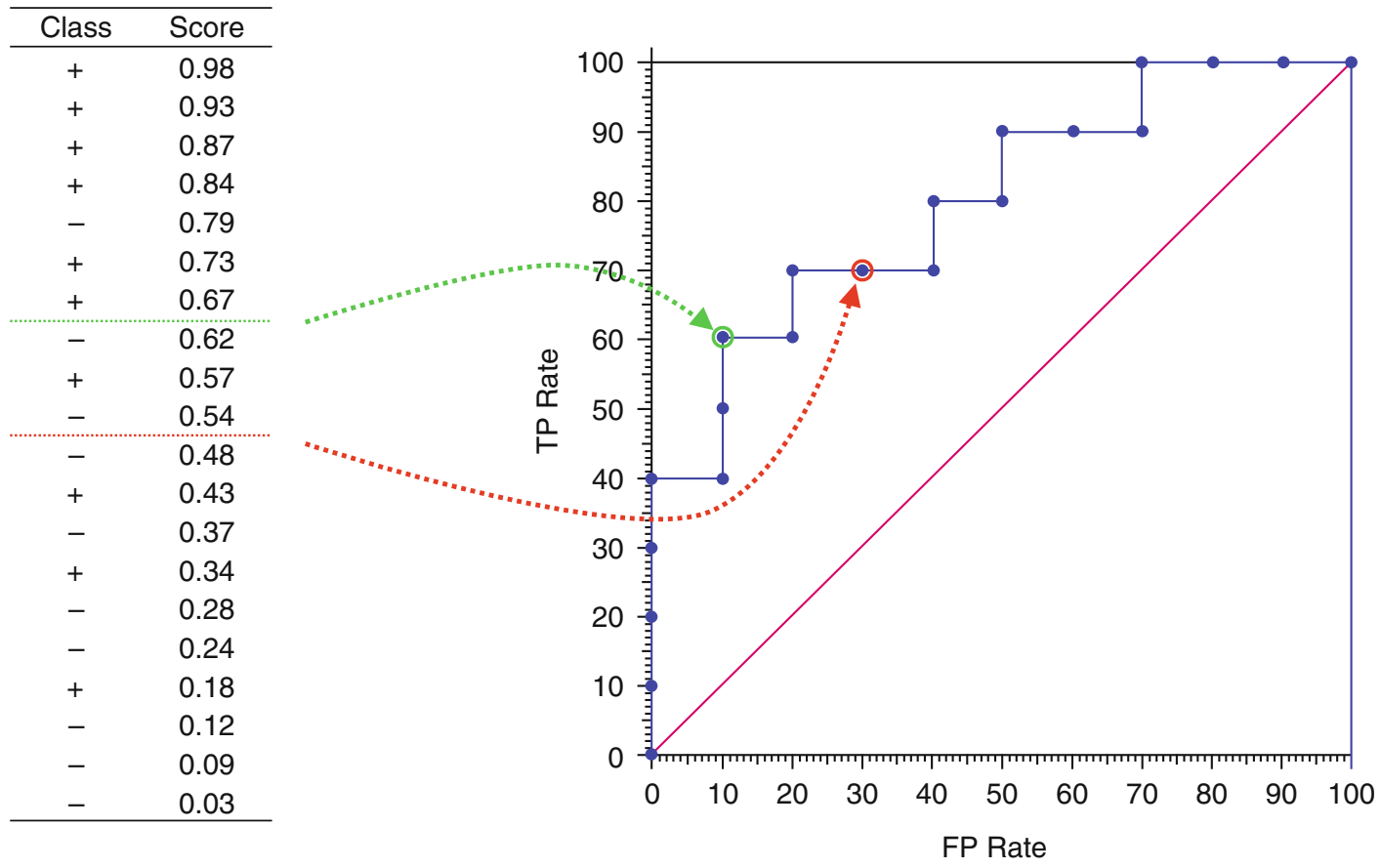

ROC Analysis, Fig. 1 The table on the left gives the scores assigned by a classifier to 10 positive and 10 negative examples. Each threshold on the classifier's score results in particular true and false positive rates, e.g., thresholding the score at 0.5 results in three misclassified positives $(t p r=0.7)$ and three misclassified negatives $(f p r=0.3)$; thresholding at 0.65 yields $t p r=0.6$ and $f p r=0.1$. Considering all possible thresholds gives the ROC curve on the right; this curve can also be constructed without explicit reference to scores, by going down the examples sorted on decreasing score and making a step up (to the right) if the example is positive (negative) and false positive rates. Varying the decision threshold from its maximal to its minimal value results in a piecewise linear curve from $(0,0)$ to $(1,1)$, such that each segment has a nonnegative slope (Fig. 1). This ROC curve is the main tool used in ROC analysis. It can be used to address a range of problems, including: (1) determining a decision threshold that minimizes > error rate or misclassification cost under given class and cost distributions; (2) identifying regions where one classifier outperforms another; (3) identifying regions where a classifier performs worse than chance; (4) obtaining calibrated estimates of the class posterior.

\section{Motivation and Background}

ROC analysis has its origins in signal detection theory (Egan 1975). In its simplest form, a detection problem involves determining the value of a binary signal contaminated with random noise. In the absence of any other information, the most sensible decision threshold would be halfway between the two signal values. If the noise distribution is zero centered and symmetric, sensitivity and specificity at this threshold have the same expected value, which means that the corresponding operating point on the ROC curve is located at the intersection with the descending diagonal $t p r+f p r=1$. However, we may wish to choose different operating points, for instance, because false negatives and false positives have different costs. In that case, we need to estimate the noise distribution.

A slight reformulation of the signal detection scenario clarifies its relevance in a machine learning setting. Instead of superimposing random noise on a deterministic signal, we can view the resulting noisy signal as coming from a - mixture distribution consisting of two compo- 


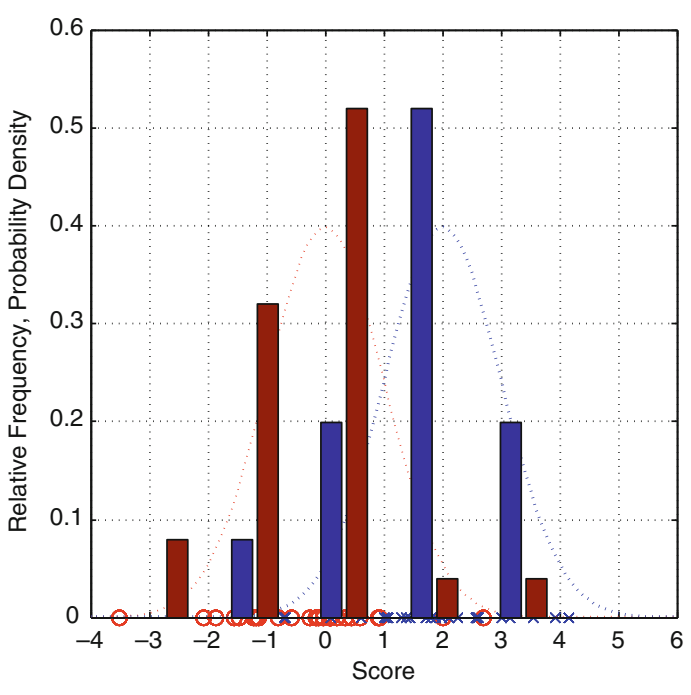

ROC Analysis, Fig. 2 (left) Artificial classifier "scores" for two classes were obtained by sampling 25 points each from two $\triangleright$ Gaussian distributions with mean 0 and 2 and unit variance. The figure shows the raw scores on the $x$-axis and normalized histograms obtained by uniform

nent distributions with different means. The detection problem is now to decide, given a received value, from which component distribution it was drawn. This is essentially what happens in a binary $\downarrow$ classification scenario, where the scores assigned by a trained classifier follow a mixture distribution with one component for each class. The random variations in the data are translated by the classifier into random variations in the scores, and the classifier's performance depends on how well the per-class score distributions are separated. Figure 2 illustrates this for both discrete and continuous distributions. In practice, empirical ROC curves and distributions obtained from a test set are discrete because of the finite resolution supplied by the test set. This resolution is further reduced if the classifier only assigns a limited number of different scores, as is the case with $\triangleright$ decision trees; the histogram example illustrates this.

\section{Solutions}

For convenience, we will assume henceforth that score distributions are discrete and that decision thresholds always fall between actual scores

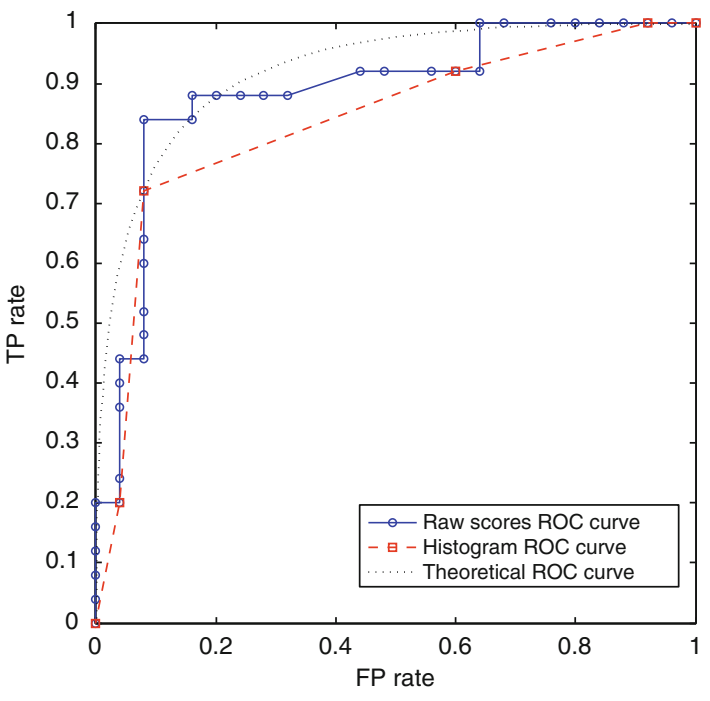

five-bin discretization (right) The jagged ROC curve was obtained by thresholding the raw scores as before. The histogram gives rise to a smoothed ROC curve with only five segments. The dotted line is the theoretical curve obtained from the true Gaussian distributions

(the results easily generalize to continuous distributions using probability density functions). There is a useful duality between thresholds and scores: decision thresholds correspond to operating points connecting two segments in the ROC curve, and actual scores correspond to segments of the ROC curve connecting two operating points. Let $f(s \mid+)$ and $f(s \mid-)$ denote the relative frequency of positive (negative) examples from a test set being assigned score $s$. (Note that $s$ itself may be an estimate of the likelihood $p(x \mid+)$ of observing a positive example with feature vector $x$. We will return to this later.)

\section{Properties of ROC Curves}

The first property of note is that the true (false) positive rate achieved at a certain decision threshold $t$ is the proportion of the positive (negative) score distribution to the right of the threshold; that is, $\operatorname{tpr}(t)=\sum_{s>t} f(s \mid+)$ and $\operatorname{fpr}(t)=$ $\sum_{s>t} f(s \mid-)$. In Fig. 2, setting the threshold at 1 using the discretized scores gives a true positive rate of 0.72 and a false positive rate of 0.08 , as can be seen by summing the bars of the histogram to the right of the threshold. Although the ROC 
curve does not display thresholds or scores, this allows us to reconstruct the range of thresholds yielding a particular operating point from the score distributions.

If we connect two distinct operating points on an ROC curve by a straight line, the slope of that line segment is equal to the ratio of positives to negatives in the corresponding score interval; that is,

$$
\begin{aligned}
\text { slope }\left(t_{1}, t_{2}\right) & =\frac{\operatorname{tpr}\left(t_{2}\right)-\operatorname{tpr}\left(t_{1}\right)}{f p r\left(t_{2}\right)-f p r\left(t_{1}\right)} \\
& =\frac{\sum_{t_{1}<s<t_{2}} f(s \mid+)}{\sum_{t_{1}<s<t_{2}} f(s \mid-)}
\end{aligned}
$$

Choosing the score interval small enough to cover a single segment of the ROC curve corresponding to score $s$, it follows that the segment has slope $f(s \mid+) / f(s \mid-)$. This can be verified in Fig. 2, e.g., the top-right segment of the smoothed curve has slope 0 because the leftmost bin of the histogram contains only negative examples. For continuous distributions, the slope of the ROC curve at any operating point is equal to the ratio of probability densities at that score.

It can happen that slope $\left(t_{1}, t_{2}\right)<\operatorname{slope}\left(t_{1}, t_{3}\right)<$ slope $\left(t_{2}, t_{3}\right)$ for $t_{1}<t_{2}<t_{3}$, which means that the ROC curve has a "dent" or concavity. This is inevitable when using raw classifier scores (unless the positives and negatives are perfectly separated), but can also be observed in the smoothed curve in the example: the rightmost bin of the histogram has a positiveto-negative ratio of 5 , while the next bin has a ratio of 13. Consequently, the two leftmost segments of the ROC curve display a slight concavity. What this means is that performance can be improved by combining those two bins, leading to one large segment with slope 9. In other words, ROC curve concavities demonstrate locally suboptimal behavior of a classifier. An extreme case of suboptimal behavior occurs if the entire curve is concave or at least below the ascending diagonal: in that case, performance can simply be improved by assigning all test instances the same score, resulting in an ROC curve that follows the ascending diagonal. A convex ROC curve is one without concavities.

\section{The AUC Statistic}

The most important statistic associated with ROC curves is the area under (ROC) curve or AUC. Since the curve is located in the unit square, we have $0 \leq A U C \leq 1$. AUC $=1$ is achieved if the classifier scores every positive higher than every negative; $A U C=0$ is achieved if every negative is scored higher than every positive. $A U C=1 / 2$ is obtained in a range of different scenarios, including: (i) the classifier assigns the same score to all test examples, whether positive or negative, and thus the ROC curve is the ascending diagonal; (ii) the per-class score distributions are similar, which results in an ROC curve close (but not identical) to the ascending diagonal; and (iii) the classifier gives half of a particular class the highest scores and the other half the lowest scores. Notice that, although a classifier with $A U C$ close to one half is often said to perform randomly, there is nothing random in the third classifier: rather, its excellent performance on some of the examples is counterbalanced by its very poor performance on some others (Sometimes a linear rescaling $2 \cdot A U C-1$ called the Gini coefficient is preferred, which has a related use in the assessment of income or wealth distributions using Lorenz curves: a Gini coefficient close to 0 means that income is approximately evenly distributed. Notice that this Gini coefficient is often called the Gini index, but should not be confused with the impurity measure used in $\triangleright$ decision tree learning).

$A U C$ has a very useful statistical interpretation: it is the expectation that a (uniformly) randomly drawn positive receives a higher score than a randomly drawn negative. It is a normalized version of the Wilcoxon-Mann-Whitney sum of ranks test, which tests the null hypothesis that two samples of ordinal measurements are drawn from a single distribution. The "sum of ranks" epithet refers to one method to compute this statistic, which is to assign each test example an integer rank according to decreasing score (the highestscoring example gets rank 1 , the next gets rank 2 , etc.); sum up the ranks of the $n^{-}$negatives, 
which we want to be high; and subtract $\sum_{i=1}^{n^{-}} i=$ $n^{-}\left(n^{-}+1\right) / 2$ to achieve 0 if all negatives are ranked first. The $A U C$ statistic is then obtained by normalizing by the number of pairs of one positive and one negative, $n^{+} n^{-}$. There are several other ways to calculate $A U C$, for instance, we can calculate, for each negative, how many positives precede it, which basically is a columnwise calculation and yields an alternative view of $A U C$ as the expected true positive rate if the operating point is chosen just before a randomly drawn negative.

\section{Identifying Optimal Points and the ROC Convex Hull}

In order to select an operating point on an ROC curve, we first need to specify the objective function we aim to optimize. In the simplest case, this will be - accuracy, the proportion of correctly predicted examples. Denoting the proportion of positives by pos, we can express accuracy as a weighted average of the true positive and true

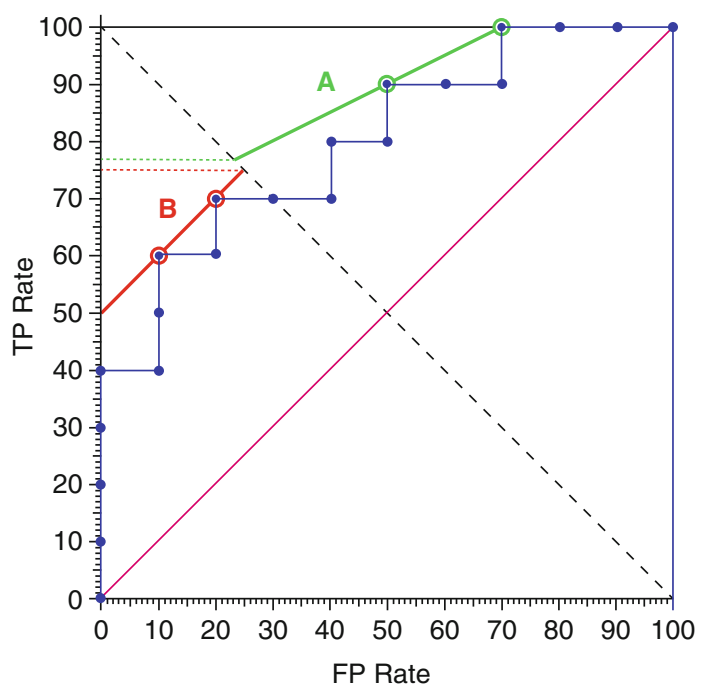

ROC Analysis, Fig. 3 (left) The slope of accuracy isometrics reflects the class ratio. Isometric $A$ has slope 1/2: this corresponds to having twice as many positives as negatives, meaning that an increase in true positive rate of $x$ is worth a $2 x$ increase in false positive rate. This selects two optimal points on the ROC curve. Isometric $\mathrm{B}$ corresponds to a uniform class distribution and selects optimal points which make fewer positive predictions. In negative rates pos $\cdot t p r+(1-p o s)(1-f p r)$. It follows that points with the same accuracy lie on a straight line with slope $a=(1-$ pos $) / p o s$; these parallel lines are the isometrics for accuracy (Peter 2003). In order to find the optimal operating point for a given class distribution, we can start with an accuracy isometric through $(0,1)$ and slide it down until it touches the ROC curve in one or more points (Fig. 3 (left)). In the case of a single point, this uniquely determines the operating point and thus the threshold. If there are several points in common between the accuracy isometric and the ROC curve, we can make an arbitrary choice or interpolate stochastically. We can read off the achieved accuracy by intersecting the accuracy isometric with the descending diagonal, on which $t p r=1-f p r$, and therefore the true positive rate at the intersection point is equal to the accuracy associated with the isometric.

We can generalize this approach to any objective function that is a linear combination of true and false positive rates. For instance, let predicting class $i$ for an instance of class $j$

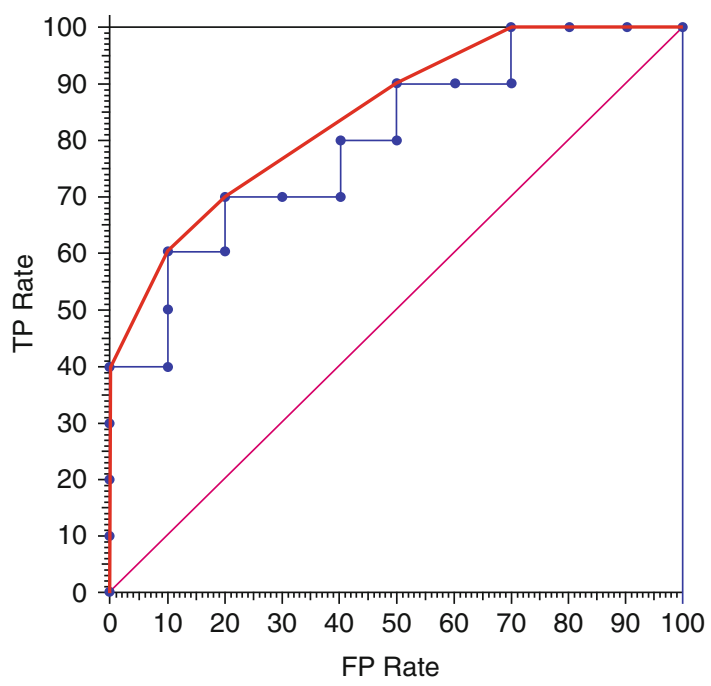

either case, the achieved accuracy can be read off on the $y$-axis after intersecting the isometric with the descending diagonal (slightly higher for points selected by A). (right) The convex hull selects those points on an ROC curve which are optimal under some class distribution. The slope of each segment of the convex hull gives the class ratio under which the two end points of the segment yield equal accuracy. All points under the convex hull are non-optimal 
incur cost $\operatorname{cost}(i \mid j)$, so, for instance, the cost of a false positive is $\operatorname{cost}(+\mid-)$ (profits for correct predictions are modeled as negative costs, e.g., $\operatorname{cost}(+\mid+)<0)$. Cost isometrics then have slope

$$
\frac{\operatorname{cost}(+\mid-)-\operatorname{cost}(-\mid-)}{\operatorname{cost}(-\mid+)-\operatorname{cost}(+\mid+)}
$$

Nonuniform class distributions are simply taken into account by multiplying the class and cost ratio, giving a single skew ratio expressing the relative importance of negatives compared to positives.

This procedure of selecting an optimal point on an ROC curve can be generalized to select among points lying on more than one curve or even an arbitrary set of points (e.g., points representing different categorical classifiers). In such scenarios, it is likely that certain points are never selected for any skew ratio; such points are said to be dominated. For instance, points on a concave region of an ROC curve are dominated. The nondominated points are optimal for a given closed interval of skew ratios and can be joined to form the convex hull of the given ROC curve or set of ROC points (Fig. 3 (right)); in multi-objective optimization, this concept is called the Pareto front. This notion of the ROC convex hull (sometimes abbreviated to $\mathrm{ROCCH}$ ) is extremely useful in a range of situations. For instance, if an ROC curve displays concavities, the convex hull represents a discretization of the scores which achieves higher AUC. Alternatively, the convex hull of a set of categorical classifiers can be interpreted as a hybrid classifier that can reach any point on the convex hull by stochastic interpolation between two neighboring classifiers (Foster and Tom 2001).

\section{Obtaining Calibrated Estimates of the Class Posterior}

Recall that each segment of an ROC curve has slope slope $(s)=f(s \mid+) / f(s \mid-)$, where $s$ is the score associated with the segment, and $f(s \mid+)$ and $f(s \mid-)$ are the relative frequencies of positives and negatives assigned score $s$. Now consider the function

$$
\begin{aligned}
\operatorname{cal}(s) & =\frac{\operatorname{pos} \cdot f(s \mid+)}{\operatorname{pos} \cdot f(s \mid+)+(1-\text { pos }) \cdot f(s \mid-)} \\
& =\frac{\operatorname{slope}(s)}{\operatorname{slope}(s)+a}
\end{aligned}
$$

with $a=(1-$ pos $) /$ pos. The calibration map $s \mapsto \operatorname{cal}(s)$ adjusts the classifier's scores to reflect the empirical probabilities observed in the test set. If the ROC curve is convex, slope $(s)$ and $\operatorname{cal}(s)$ are monotonically nonincreasing with decreasing $s$, and thus replacing the scores $s$ with $\operatorname{cal}(s)$ does not change the ROC curve (other than merging neighboring segments with different scores but the same slope into a single segment).

Consider $\downarrow$ decision trees as a concrete example. Once we have trained (and possibly pruned) a tree, we can obtain a score in each leaf $l$ by taking the proportion of positive training examples in that leaf: $\operatorname{score}(l)=p(+\mid l) /(p(+\mid l)+p(-\mid l))$. Each leaf of the tree then gives rise to a different segment of the ROC curve, which, by the nature of how the scores were calculated, will be convex. Furthermore, we have that $\operatorname{cal}(\operatorname{score}(l))=$ score $(l)$, which means that the tree produces posterior probabilities that are perfectly calibrated with respect to the training set. If we anticipate changes in class distribution, we may choose to calibrate with a different $a$. For example, if we use $a=1$, the calibrated scores $\operatorname{cal}(\operatorname{score}(l))$ are adjusted for a uniform prior.

If the ROC curve is not convex, the mapping $s \mapsto \operatorname{cal}(s)$ is not monotonic; while the scores $\operatorname{cal}(s)$ would lead to improved performance on the data from which the ROC curve was derived, this is very unlikely to generalize to other data and thus leads to $>$ overfitting. This is why, in practice, a less drastic calibration procedure involving the convex hull is applied (Tom and Alexandru 2007). Let $s_{1}$ and $s_{2}$ be the scores associated with the start and end segments of a concavity, i.e., $s_{1}>s_{2}$ and slope $\left(s_{1}\right)<$ slope $\left(s_{2}\right)$. Let slope $\left(s_{1} s_{2}\right)$ denote the slope of the line segment of the convex hull that repairs this concavity, which implies slope $\left(s_{1}\right)<$ slope $\left(s_{1} s_{2}\right)<\operatorname{slope}\left(s_{2}\right)$. The calibration map will then map any score in the interval $\left[s_{1}, s_{2}\right]$ to slope $\left(s_{1} s_{2}\right) /\left(\operatorname{slope}\left(s_{1} s_{2}\right)+1\right)$ (Fig. 4). 


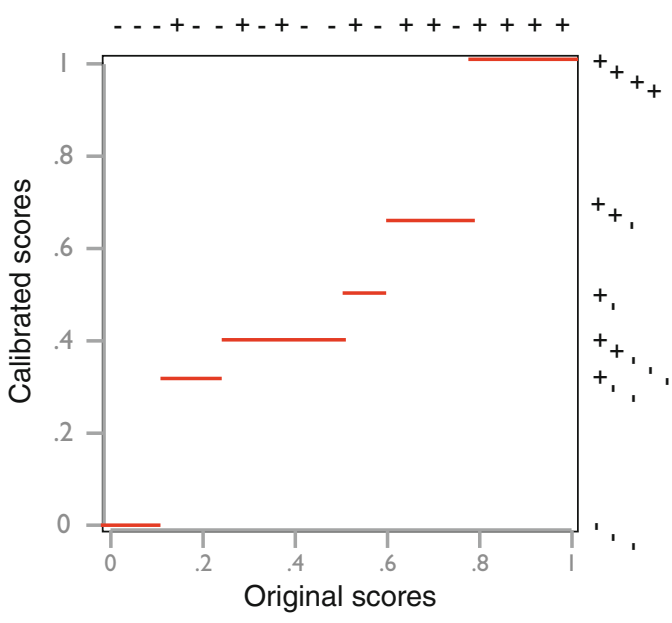

ROC Analysis, Fig. 4 The piecewise constant calibration map derived from the convex hull in Fig. 3. The original score distributions are indicated at the top of the figure, and the calibrated distributions are on the right. We can clearly see the combined effect of binning the scores and redistributing them over the interval $[0,1]$

This ROC-based calibration procedure, which is also known as isotonic regression (Barbara and Charles 2002), not only produces calibrated probability estimates but also improves AUC. This is in contrast with other calibration procedures such as logistic calibration which do not bin the scores and therefore do not change the ROC curve. ROC-based calibration can be shown to achieve the lowest Brier score (Glenn 1950), which measures the mean squared error in the probability estimates as compared with the ideal probabilities ( 1 for a positive and 0 for a negative), among all probability estimators that do not reverse pairwise rankings. On the other hand, being a nonparametric method, it typically requires more data than parametric methods in order to estimate the bin boundaries reliably. See - Classifier Calibration for further details.

\section{Future Directions}

ROC analysis in its original form is restricted to binary classification, and its extension to more than two classes gives rise to many open problems. $c$-class ROC analysis requires $c(c-1)$ dimensions, in order to distinguish each possible misclassification type. Srinivasan proved that basic concepts such as the ROC polytope and its linearly interpolated convex hull generalize to the $c$-class case (Ashwin 1999). In theory, the volume under the ROC polytope can be employed for assessing the quality of a multi-class classifier (César et al. 2003), but this volume is hard to compute as - unlike the two-class case, where the segments of an ROC curve can simply be enumerated in $O(n \log n)$ time by sorting the $n$ examples on their score (Tom 2006; Peter 2004) - there is no simple way to enumerate the ROC polytope. Mossman considers the special case of three-class ROC analysis, where for each class the two possible misclassifications are treated equally (a so-called oneversus-rest scenario) (Douglas 1999). Hand and Till propose the average of all one-versus-rest AUCs as an approximation of the area under the ROC polytope (David and Robert 2001). Various algorithms for minimizing a classifier's misclassification costs by reweighting the classes are considered in Nicolas and Peter (2003) and Chris et al. (2008).

Other research directions include the explicit visualization of misclassification costs (Chris and Robert 2006) and using ROC analysis to study the behavior of machine learning algorithms and the relations between machine learning metrics (Johannes and Peter 2005).

\section{Cross-References}
- Accuracy
- Classification
- Classifier Calibration
- Confusion Matrix
- Cost-Sensitive Learning
- Error Rate
$>$ False Negative
- False Positive
- Gaussian Distribution
- Posterior Probability
- Precision
- Prior Probability
- Recall 
- Sensitivity

- Specificity

- True Negative

- True Positive

\section{Recommended Reading}

Bourke C, Deng K, Scott S, Schapire R, Vinodchandran NV (2008) On reoptimizing multi-class classifiers. Mach Learn 71(2-3):219-242

Brier G (1950) Verification of forecasts expressed in terms of probabilities. Mon Weather Rev 78:1-3

Drummond C, Holte R (2006) Cost curves: an improved method for visualizing classifier performance. Mach Learn 65(1):95-130

Egan J (1975) Signal detection theory and ROC analysis. Series in cognitition and perception. Academic Press, New York

Fawcett T (2006) An introduction to ROC analysis. Patt Recognit Lett 27(8):861-874

Fawcett T, Niculescu-Mizil A (2007) PAV and the ROC convex hull. Mach Learn 68(1):97-106

Ferri C, Hernández-Orallo J, Salido M (2003) Volume under the ROC surface for multi-class problems. In: Proceedings of the fourteenth European conference on machine learning, Cavtat, pp 108-120

Flach P (2003) The geometry of ROC space: understanding machine learning metrics through ROC isometrics. In: Proceedings of the twentieth international conference on machine learning (ICML 2003), Washington, DC, pp 194-201

Flach P (2004) The many faces of ROC analysis in machine learning, July 2004. ICML-04 Tutorial. Notes available from http://www.cs.bris.ac. uk/ flach/ICML04tutorial/index.html

Fuernkranz J, Flach P (2005) ROC 'n' Rule learning - towards a better understanding of covering algorithms. Mach Learn 58(1):39-77

Hand D, Till R (2001) A simple generalization of the area under the ROC curve to multiple class classification problems. Mach Learn 45(2):171-186

Lachiche N, Flach P (2003) Improving accuracy and cost of two-class and multi-class probabilistic classifiers using ROC curves. In: Proceedings of the twentieth international conference on machine learning (ICML'03), Washington, DC, pp 416-423

Mossman D (1999) Three-way ROCs. Med Decis Mak 19:78-89

Provost F, Fawcett T (2001) Robust classification for imprecise environments. Mach Learn 42(3): 203-231

Srinivasan A (1999) Note on the location of optimal classifiers in n-dimensional ROC space. Technical report PRG-TR-2-99, Oxford University Computing Laboratory, Oxford

Zadrozny B, Elkan C (2002) Transforming classifier scores into accurate multiclass probability es- timates. In: Proceedings of the 8th ACM SIGKDD international conference on Knowledge discovery and data mining, Edmonton. ACM, pp 694-699

\section{ROC Convex Hull}

The convex hull of an $>$ ROC curve is a geometric construction that selects the points on the curve that are optimal under some class and cost distribution. It is analogous to the Pareto front in multiobjective optimization. See $>$ ROC Analysis.

\section{ROC Curve}

The ROC curve is a plot depicting the trade-off between the $>$ true positive rate and the $>$ false positive rate for a classifier under varying decision thresholds. See $>$ ROC Analysis.

\section{Rotation Forests}

Rotation Forests is an $>$ ensemble learning technique. It is similar to the $>$ Random Forests approach to building decision tree ensembles. In the first step, the original feature set is split randomly into $K$ disjoint subsets. Next, $>$ principal components analysis is used to extract $n$ principal component dimensions from each of the $K$ subsets. These are then pooled, and the original data projected linearly into this new feature space. A tree is then built from this data in the usual manner. This process is repeated to create an ensemble of trees, each time with a different random split of the original feature set.

As the tree learning algorithm builds the classification regions using hyperplanes parallel to the feature axes, a small rotation of the axes may lead to a very different tree. The effect of rotating the axes is that classification regions of high accuracy can be constructed with far fewer trees than in $>$ Bagging and $>$ Adaboost. 


\section{RSM}

- Random Subspace Method

\section{Rule Learning}

Johannes Fürnkranz

Knowledge Engineering Group, TU Darmstadt,

Darmstadt, Deutschland

Department of Information Technology,

University of Leoben, Leoben, Austria

\begin{abstract}
Informally, rule learning denotes all algorithms that learn or discover patterns in data, which are formulated in the form of a $>$ rule. These can be predictive (e.g., > classification rules) or descriptive rules (e.g., $\checkmark$ association rules or $\$$ supervised descriptive rule induction). Consequently, the learning algorithms typically differ in the type of search they use for finding these rules in the search space. Exhaustive search is more common in descriptive rule mining, whereas heuristic search using a variety of quality criteria is more commonly used in predictive rule learning. An overview of the field can be found in Fürnkranz et al. (2012).
\end{abstract}

\section{Learning Individual Rules}

Conceptually, rule learning may be viewed as a search in the space of possible $>$ rules. The first algorithms, such as the candidate elimination algorithm, aimed at identifying the $\vee$ version space of all complete and consistent rules (Mitchell 1982). Association rule discovery algorithms look for all rules that satisfy certain constraints, typically all rules with a minimum coverage and a minimum support. Most flexible are algorithms that use heuristic search for optimizing given quality criteria. Such algorithms are also often used in $>$ supervised descriptive rule induction. procedure FindBESTRuLE(Examples,BestRule)

Input: Examples, a set of positive and negative examples for a class $c$.

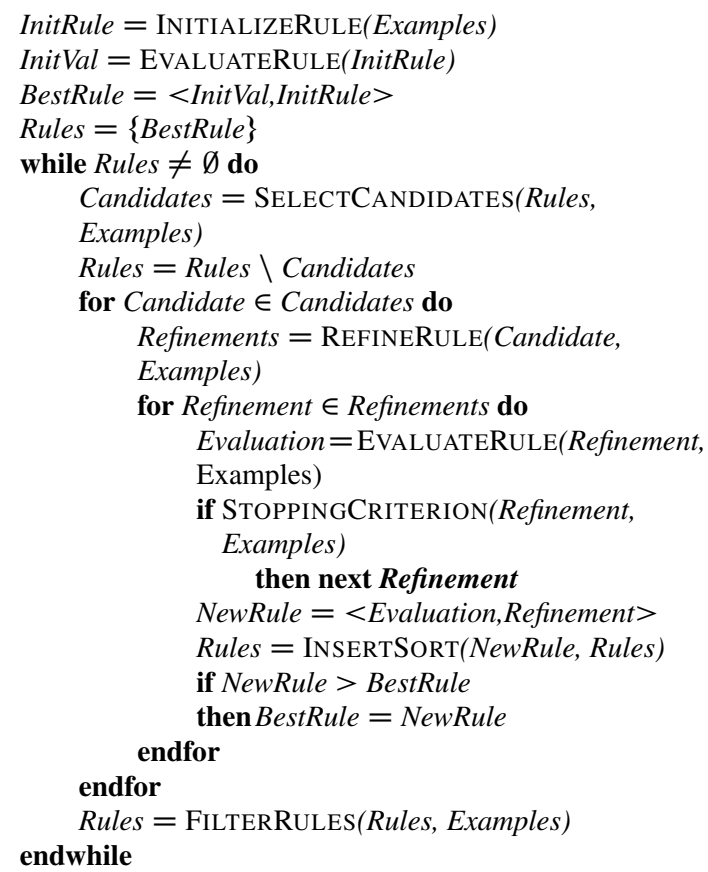

Output: BestRule

FINDBESTRULE is a prototypical algorithm that searches for a rule which optimizes a given quality criterion defined in EVALUATERULE. The value of this heuristic function is the higher the more positive and the less negative examples are covered by the candidate rule. FindBESTRULE maintains Rules, a sorted list of candidate rules, which is initialized by the procedure INITIALIZERULE. New rules will be inserted in appropriate places (INSERTSORT), so that Rules will always be sorted in decreasing order of the heuristic evaluations of the rules. At each cycle, SELECTCANDIDATES selects a subset of these candidate rules, which are then refined using the refinement operator REFINERULE. Each refinement is evaluated and inserted into the sorted Rules list unless the STOPPINGCRITERION prevents this. If the evaluation of the NewRule is better than the best rule found previously, BestRule is set to NewRule. FILTERRULES selects the subset of the 
ordered rule list that will be used in subsequent iterations. When all candidate rules have been processed, the best rule will be returned.

Different choices of these functions allow the definition of different biases for the separate-and-conquer learner. The search bias is defined by the choice of a search strategy (INITIALIZERULE and REFINERULE), a search algorithm (SELECTCANDIDATES and FilterRules), and a search heuristic (EvaluateRule). The refinement operator REFINERULE constitutes the language bias of the algorithm. An overfitting avoidance bias can be implemented via some STOPPINGCRITERION and/or in a post-processing phase.

For example, InItializeRule and REFINERULE may be defined so that they realize a top-down (general-to-specific), a bottom-up (specific-to-general), or a bidirectional search. Exhaustive breadth-first, depth-first, or best-first searches can be realized by appropriate choices of EVALUATERULE and no filtering or candidate selection. FilterRules can, e.g., be used to realize a hill-climbing or $>$ beam search by maintaining only the best or the BeamWidth best rules. Evolutionary algorithms and stochastic local search can also be easily realized.

The most common algorithm for finding the best rule is a top-down hill-climbing algorithm. It basically constructs a rule by consecutively adding conditions to the rule body so that a given quality criterion is greedily optimized. This constitutes a simple greedy hill-climbing algorithm for finding a local optimum in the hypothesis space defined by the feature set. INITIALIZERULE will thus return the most general rule, the rule with the body $\{$ true\}, and REFINERULE will return all possible extensions of the rule by a single condition. FILTERRULES will only let the best refinement pass for the next iteration, so that SELECTCANDIDATES will always have only one choice. The search heuristic, the StoppingCriterion, and the post-processing are discussed in the next sections.

\section{Rule Learning Heuristics}

The goal of rule learning is to find a rule or a $\checkmark$ rule set that is as complete and consistent as possible. Thus, each rule should cover as many positive examples and as few negative examples as possible. A few important ones are (assume that $p$ out of $P$ positive examples and $n$ out of $N$ negative examples are covered by the rule):

Laplace estimate $\left(\mathrm{Lap}=\frac{p+1}{p+n+2}\right)$ computes the fraction of positive examples in all covered examples, where each class is initialized with one virtual example in order to penalize rules with low coverage.

$m$-Estimate $\left(\mathrm{m}=\frac{p+m \cdot P /(P+N)}{p+n+m}\right)$ is a generalization of the Laplace estimate which uses $m$ examples for initialization, which are distributed according to the class distribution in the training set (Cestnik 1990).

Information gain $\left(\mathrm{ig}=p \cdot\left(\log _{2} \frac{p}{p+n}-\right.\right.$ $\log _{2} \frac{p^{\prime}}{p^{\prime}+n^{\prime}}$, where $p^{\prime}$ and $n^{\prime}$ are the number of positive and negative examples covered by the rule's predecessor) is Quinlan's (1990) adaptation of the information gain heuristic used for decision tree learning. The main difference is that this only focuses on a single branch (a rule), whereas the decision tree version tries to optimize all branches simultaneously.

correlation and $\chi^{2}\left(\operatorname{corr}=\frac{p(N-n)-(P-p) n}{\sqrt{P N(p+n)(P-p+N-n)}}\right)$ computes the four-field correlation of covered/uncovered positive/negative examples. It is equivalent to a $\chi^{2}$ statistic $\left(\chi^{2}=\right.$ $\left.(P+N) \operatorname{corr}^{2}\right)$.

An exhaustive overview and theoretical comparison of various search heuristics in coverage space, a variant of ROC space can be found in Fürnkranz and Flach (2005).

\section{Overfitting Avoidance}

It is trivial to find a rule set that is complete and consistent on the training data. To achieve this, one only needs to convert each positive example into a rule. Each of these rules is consistent (provided the data set is not inconsistent), and collectively they cover the entire example set (completeness). However, this is clearly a bad case of $>$ overfitting because the theory will not generalize to new positive examples. 
Overfitting is to some extent handled by the search heuristics described above, but most algorithms use additional $>$ pruning techniques. One can discriminate between pre-pruning techniques, where a separate criterion is used to filter out unpromising rules. For example, $\mathrm{CN} 2$ computes the likelihood ratio statistic lrs $=2 \cdot\left(p \log \frac{p}{e_{p}}+n \log \frac{n}{e_{n}}\right)$, where $e_{p}=(p+$ $n) \frac{P}{P+N}$ and $e_{n}=(p+n) \frac{N}{P+N}=(p+n)-e_{p}$ are the number of positive and negative examples one could expect if the $p+n$ examples covered by the rule were distributed in the same way as the $P+N$ examples in the full data set. This statistic follows a $\chi^{2}$ distribution, which allows to filter out rules for which the distribution of the covered examples is not statistically significantly different from the distribution of examples in the full data set. Other pre-pruning criteria are simple thresholds that define a minimum acceptable value for the search heuristic or FOIL's $>$ minimum description length criterion that relates the length of a rule to the number of examples it covers.

However, it can be shown experimentally that CN2 or FoIL still has a tendency to overfit the data. Instead, state-of-the-art algorithms postprune a rule right after it has been learned. For this purpose, one-third of the training data are reserved for pruning. After a rule has been learned, its accuracy is greedily simplified on the pruning set. Simplifications can be the deletion of the last condition, a final sequence of conditions, or an arbitrary condition of the rule. If the simplification does not decrease the accuracy of the rule on the pruning set, it will be performed. This so-called incremental reduced error pruning algorithm (Fürnkranz and Widmer 1994) is used in the rule learning algorithm RIPPER.

A survey and experimental comparison of pruning techniques for rule learning can be found in Fürnkranz (1997).

\section{Learning Rule Sets}

In many cases, rule learning is used for solving a classification problem via the induction of $\mathrm{a} \triangleright$ rule set or a $\triangleright$ decision list. In these cases, individual rules are learned as above but then combined to form a theory that is able to classify all examples. The principal approach is the socalled $>$ covering or $>$ separate-and-conquer algorithm, which learns one rule at a time, successively removing the covered examples. Individual algorithms within this framework differ primarily in the way they learn single rules.

An obvious generalization of covering is to not entirely remove covered examples but to reduce their example $>$ weights, thus decreasing their importance in subsequent iterations (see, e.g., the SLIPPER algorithm (Cohen and Singer 1999)).

Rules can also be learned by alternative strategies. There have been numerous proposals, and we can only mention the most influential. Each path from the root to a leaf of a $>$ decision tree corresponds to a rule and so rules can be learned by first learning a decision tree and then postprocessing it (see, e.g., the C4.5RULES algorithm, (Quinlan 1993)). It is also possible to use the $>$ Apriori algorithm for an exhaustive search for classification rules and to use a subsequent covering algorithm to combine the rules into a rule set (see, e.g., the CBA algorithm (Liu et al. 1998)). RISE (Domingos 1996) combines bottom-up generalization with $\triangleright$ nearest neighbor algorithms to learn a theory via "conquering without separating."

\section{Well-Known Rule Learning Algorithms}

AQ can be considered as the original covering algorithm. Its original version was conceived by Ryszard Michalski in the 1960s (Michalski 1969), and numerous versions and variants of the algorithm appeared subsequently in the literature. AQ uses a top-down beam search for finding the best rule. It does not search all possible specializations of a rule but only considers refinements that cover a particular example, the so-called seed example. This idea is basically the same as the use of a $>$ bottom clause in $>$ inductive logic programming.

CN2 (Clark and Niblett 1989; Clark and Boswell 1991) employs a beam search guided 
by the Laplace or $m$-estimates, and the abovementioned likelihood ratio significance test to fight overfitting. It can operate in two modes, one for learning $>$ rule sets (by modeling each class independently) and one for learning $\checkmark$ decision lists.

FoIL (Quinlan 1990) was the first relational learning algorithm that received attention beyond the field of $>$ inductive logic programming. It learns a concept with the covering loop and learns individual concepts with a top-down refinement operator, guided by information gain. The main difference to previous systems is that FoIL allowed the use of first-order background knowledge. Instead of only being able to use tests on single attributes, FOIL could employ tests that compute relations between multiple attributes and could also introduce new variables in the body of a rule.

RIPPER was the first rule learning system that effectively countered the overfitting problem via incremental reduced error pruning, as described above. It also added a post-processing phase for optimizing a rule set in the context of other rules. The key idea is to remove one rule out of a previously learned rule set and try to relearn it not only in the context of previous rules (as would be the case in the regular covering rule) but also in the context of subsequent rules. RIPPER is still state of the art in inductive rule learning. A freely accessible re-implementation can be found in the WEKA machine learning library under the name of JRIP.

Opus (Webb 1995) was the first rule learning algorithm to demonstrate the feasibility of a full exhaustive search through all possible rule bodies for finding a rule that maximizes a given quality criterion (or heuristic function). The key idea is the use of ordered search that prevents that a rule is generated multiple times. This means that even though there are $l$ ! different orders of the conditions of a rule of length $l$, only one of them can be taken by the learner for finding this rule. In addition, OPUS uses several techniques that prune significant parts of the search space, so that this search method becomes feasible. Follow-up work has shown that this technique is also an efficient alternative for $>$ association rule discovery, provided that the database to mine fits into the memory of the learning system.

CBA was one of the first and best-known algorithms that employed association rule learning algorithms for learning predictive rules (Liu et al. 1998). In its simplest version, the algorithm selects the final rule sets by sorting all class association rules according to confidence and incrementally adding rules to the final set until all examples are covered or the quality of the rule set decreases.

\section{Cross-References}

\author{
Association Rule \\ - Classification Rule \\ - Covering Algorithm \\ Decision List \\ > Decision Lists and Decision Trees \\ $\checkmark$ Rule Set \\ Supervised Descriptive Rule Induction
}

\section{Recommended Reading}

Cestnik B (1990) Estimating probabilities: a crucial task in machine learning. In: Aiello L (ed) Proceedings of the 9th European conference on artificial intelligence (ECAI-90). Pitman, Stockholm, pp 147150

Clark P, Boswell R (1991) Rule induction with CN2: some recent improvements. In: Proceedings of the 5th European working session on learning (EWSL91). Springer, Porto, pp 151-163

Clark P, Niblett T (1989) The CN2 induction algorithm. Mach Learn 3(4):261-283

Cohen WW, Singer Y (1999) A simple, fast, and effective rule learner. In: Proceedings of the 16th national conference on artificial intelligence (AAAI99). AAAI/MIT Press, Menlo Park, pp 335-342

Domingos P (1996) Unifying instance-based and rulebased induction. Mach Learn 24:141-168

Fürnkranz J (1997) Pruning algorithms for rule learning. Mach Learn 27(2):139-171. http://www.ke. informatik.tu-darmstadt.de/ juffi/publications/mlj97. pdf

Fürnkranz J, Flach PA (2005) ROC 'n' rule learning - towards a better understanding of covering algorithms. Mach Learn 58(1):39-77. doi:10.1007/s10994-005-5011-x. http://www.cs. bris.ac.uk/ flach/papers/furnkranz-flach-mlj.pdf 
Fürnkranz J, Widmer G (1994) Incremental reduced error pruning. In: Cohen WW, Hirsh H (eds) Proceedings of the 11th international conference on machine learning (ML-94). Morgan Kaufmann, New Brunswick, pp 70-77. http://www.ke.informatik.tudarmstadt.de/ juffi/publications/ml-94.ps.gz

Fürnkranz J, Gamberger D, Lavrač N (2012) Foundations of rule learning. Springer. doi:10.1007/9783-540-75197-7. ISBN 978-3-540-75196-0. http:// www.springer.com/978-3-540-75196-0

Liu B, Hsu W, Ma Y (1998) Integrating classification and association rule mining. In: Agrawal R, Stolorz P, Piatetsky-Shapiro G (eds) Proceedings of the 4th international conference on knowledge discovery and data mining (KDD-98), New York, pp 80-86

Michalski RS (1996) On the quasi-minimal solution of the covering problem. In: Proceedings of the 5th international symposium on information processing (FCIP-69), vol A3 (Switching circuits), Bled, pp 125-128

Mitchell TM (1982) Generalization as search. Artif Intell 18(2):203-226

Quinlan JR (1990) Learning logical definitions from relations. Mach Learn 5:239-266

Quinlan JR (1993) C4.5: programs for machine learning. Morgan Kaufmann, San Mateo

Webb GI (1995) OPUS: an efficient admissible algorithm for unordered search. J Artif Intell Res 5: 431-465

\section{Rule Set}

\section{Johannes Fürnkranz}

Knowledge Engineering Group, TU Darmstadt,

Darmstadt, Deutschland

Department of Information Technology,

University of Leoben, Leoben, Austria

\section{Abstract}

A rule set is a collection of individual > classification rules that collectively form a classifier. In contrast to a $\triangleright$ decision list, the rules in the set do not have an inherent order, and all rules in the set have to be tried for deriving a prediction for an example.

\section{Discussion}

This may cause two types of problems that have to be resolved with additional algorithms:
Multiple rules fire: More than one rule can fire on a single example, and these rules can make contradicting predictions. This type of conflict is typically resolved by preferring rules that cover a higher fraction of training examples of their class (typically estimated with Laplace correction, see $>$ rule learning). This is equivalent to converting the rule set into a decision list that is ordered according to this evaluation heuristic. More elaborate tie-breaking schemes, such as using a Naive Bayes algorithm, or inducing a separate rule set for handling these conflicts (double induction (Lindgren and Boström 2004)), have also been tried.

No rule fires: It may also occur that no rule fires for a given example. Such cases are typically handled via a so-called default rule, which typically predicts the majority class. Again, a more complex algorithm, such as trying to find the closest rule (rule stretching (Eineborg and Boström 2001)), has been proposed.

A rule set that only contains rules for a single class, as is the result of $>$ concept learning problems, typically contains an implicit default rule for the other class (very much like a Prolog program). If all rules are conjunctive, such rule sets may be interpreted as a definition in disjunctive normal form for this class.

\section{Cross-References}

$$
\begin{aligned}
& \text { - Classification Rule } \\
& \text { Decision List } \\
& \text { - Disjunctive Normal Form } \\
& \text { - Rule Learning }
\end{aligned}
$$

\section{Recommended Reading}

Eineborg M, Boström H (2001) Classifying uncovered examples by rule stretching. In: Rouveirol C, Sebag $\mathrm{M}$ (eds) Proceedings of the eleventh international conference on inductive logic programming (ILP01), Strasbourg. Springer, pp 41-50

Lindgren T, Boström H (2004) Resolving rule conflicts with double induction. Intell Data Anal 8(5): 457468 